\title{
Asymptotic stability of solitons for the Benjamin-Ono equation
}

Carlos E. Kenig and Yvan Martel

\begin{abstract}
In this paper, we prove the asymptotic stability of the family of solitons of the Benjamin-Ono equation in the energy space. The proof is based on a Liouville property for solutions close to the solitons for this equation, in the spirit of [17], [19]. As a corollary of the proofs, we obtain the asymptotic stability of exact multi-solitons.
\end{abstract}

\section{Introduction}

We consider the Benjamin-Ono equation (BO)

$$
u_{t}+\mathcal{H} u_{x x}+u u_{x}=0, \quad(t, x) \in \mathbb{R} \times \mathbb{R},
$$

where $\mathcal{H}$ denotes the Hilbert transform

$$
\mathcal{H} u(x)=\frac{1}{\pi} \text { p.v. } \int_{-\infty}^{+\infty} \frac{u(y)}{y-x} d y=\frac{1}{\pi} \lim _{\varepsilon \rightarrow 0} \int_{|y-x|>\varepsilon} \frac{u(y)}{y-x} d y .
$$

Note that with this notation, $\int u_{x} \mathcal{H} u=\int\left|D^{\frac{1}{2}} u\right|^{2}=\|u\|_{\dot{H}^{\frac{1}{2}}}^{2}$.

The Cauchy problem for (1.1) is globally well-posed in $H^{s}$, for any $s \geq 0$ (see Tao [27] for $s \geq 1$ and Ionescu and Kenig [11] for the case $s \geq 0$, see also Burq and Planchon [5] for the case $s>\frac{1}{4}$ ). Moreover, for solutions in the energy space $H^{\frac{1}{2}}$ the following quantities are invariant

$$
\int u^{2}(t, x) d x=\int u^{2}(0, x) d x, E(t)=\int\left(u_{x} \mathcal{H} u-\frac{1}{3} u^{3}\right)(t, x) d x=E(0) .
$$

2000 Mathematics Subject Classification: 35Q53, 35Q51, 35B40.

Keywords: Benjamin-Ono equation, soliton, asymptotic stability, Liouville theorem. 
Recall the scaling and translation invariances of equation (1.1)

$$
\begin{aligned}
& \text { if } u(t, x) \text { is solution then } \\
& \forall c>0, x_{0} \in \mathbb{R}, v(t, x)=c u\left(c^{2} t, c\left(x-x_{0}\right)\right) \text { is also solution. }
\end{aligned}
$$

We call soliton any travelling wave solution $u(t, x)=Q_{c}\left(x-x_{0}-c t\right)$, where $c>0, x_{0} \in \mathbb{R}$, and $Q_{c}(x)=c Q(c x)$ solves:

$$
-\mathcal{H} Q^{\prime}+Q-\frac{1}{2} Q^{2}=0, \quad Q \in H^{\frac{1}{2}}, \quad Q>0 .
$$

It is known that there is a unique (up to translations) solution of (1.5), which is

$$
Q(x)=\frac{4}{1+x^{2}} .
$$

(see Benjamin [2] and Amick and Toland [1] for the uniqueness statement). This solution is stable (see Bennet et al. [3] and Weinstein [31]) in the following sense.

Stability of soliton in the energy space ([3], [31]). There exist $C, \alpha_{0}>0$ such that if $u_{0} \in H^{\frac{1}{2}}$ satisfies $\left\|u_{0}-Q\right\|_{H^{\frac{1}{2}}}=\alpha \leq \alpha_{0}$ then the solution $u(t)$ of (1.1) with $u(0)=u_{0}$ satisfies

$$
\sup _{t \in \mathbb{R}} \inf _{y \in \mathbb{R}}\|u(t)-Q(.-y)\|_{H^{\frac{1}{2}}} \leq C \alpha .
$$

See a sketch of proof of this result in Section 5.1.

The main result of this paper is the asymptotic stability of the family of solitons of (1.1). Then, we consider the multisoliton case (see Section 5).

Theorem 1. (Asymptotic stability of solitons in the energy space). There exist $C, \alpha_{0}>0$ such that if $u_{0} \in H^{\frac{1}{2}}$ satisfies $\left\|u_{0}-Q\right\|_{H^{\frac{1}{2}}}=\alpha \leq \alpha_{0}$, then there exists $c^{+}>0$ with $\left|c^{+}-1\right| \leq C \alpha$ and a $C^{1}$ function $\rho(t)$ such that the solution $u(t)$ of (1.1) with $u(0)=u_{0}$ satisfies

$$
\begin{aligned}
& u(t, .+\rho(t)) \rightarrow Q_{c^{+}} \text {in } H^{\frac{1}{2}} \text { weak, }\left\|u(t)-Q_{c^{+}}(.-\rho(t))\right\|_{L^{2}\left(x>\frac{t}{10}\right)} \rightarrow 0, \\
& \rho^{\prime}(t) \rightarrow c^{+} \text {as } t \rightarrow+\infty .
\end{aligned}
$$

The proof of Theorem 1 is based on the following rigidity result.

Theorem 2. (Nonlinear Liouville property). There exist $C, \alpha_{0}>0$ such that if $u_{0} \in H^{\frac{1}{2}}$ satisfies $\left\|u_{0}-Q\right\|_{H^{\frac{1}{2}}}=\alpha \leq \alpha_{0}$ and if the solution $u(t)$ of (1.1) with $u(0)=u_{0}$ satisfies for some function $\rho(t)$

$$
\forall \varepsilon>0, \exists A_{\varepsilon}>0 \text {, s.t. } \forall t \in \mathbb{R}, \quad \int_{|x|>A_{\varepsilon}} u^{2}(t, x+\rho(t)) d x<\varepsilon,
$$

then there exist $c_{1}>0, x_{1} \in \mathbb{R}$, such that

$$
u(t, x)=Q_{c_{1}}\left(x-x_{1}-c_{1} t\right), \quad\left|c_{1}-1\right|+\left|x_{1}\right| \leq C \alpha .
$$


Remark 1. In Theorem 1, the convergence of $u(t)$ to $Q_{c^{+}}$as $t \rightarrow+\infty$ is obtained strongly in $L^{2}$ in the region $x>\frac{t}{10}$. The value $\frac{1}{10}$ is somewhat arbitrary, the result holds for $x>\varepsilon t$, for any $\varepsilon>0$, provided $\alpha_{0}=\alpha_{0}(\varepsilon)>0$ is small enough. Note that this result is optimal in $L^{2}$ since $u(t)$ could contain other small (and then slow) solitons and since in general $u(t)$ does not go to 0 in $L^{2}$ for $x<0$. For example, if $\left\|u(t)-Q_{c^{+}}(.-\rho(t))\right\|_{H^{\frac{1}{2}(\mathbb{R})}} \rightarrow 0$ as $t \rightarrow+\infty$, then $E(u)=E\left(Q_{c^{+}}\right)$and $\int u^{2}=\int Q_{c^{+}}^{2}$ and so by the variational characterization of $Q(x)$ (see [31]), $u(t)=Q_{c^{+}}\left(x-x_{0}-c^{+} t\right)$ is exactly a soliton.

Under the assumptions of Theorem 1, we expect strong convergence in $H^{\frac{1}{2}}$ to be true as well in the same local sense $(x>\varepsilon t)$. This could require some more analysis.

By the methods of this paper, we are able to obtain the following weaker result (Section 4.3)

$$
\lim _{t \rightarrow+\infty} \int_{t}^{t+1}\left\|u(s, .+\rho(s))-Q_{c^{+}}\right\|_{H_{l o c}^{\frac{1}{2}}}^{2} d s=0 .
$$

Recall that the first result of asymptotic stability of solitons for generalized KdV equations was proved by Pego and Weinstein [24] in weighted spaces. The discussion p. 308 in [24] justifies the emergence of a pure dominant wave going to the right as time goes to $+\infty$, whereas small amplitude solitary waves and dispersion have small or negative speeds. The same discussion applies to the Benjamin-Ono equation. This is reflected in the monotonicity arguments we use to prove Theorem 1 (see Proposition 1). These arguments are in fact inspired by the proof of Kato's "local smoothing" effect for the generalized KdV equation, [12].

The proof of Theorem 1 follows the approach of [16], [17], concerning the case of the generalized $\mathrm{KdV}$ equations, where the asymptotic stability of the family of solitons is deduced from a Liouville type theorem such as Theorem 2. Moreover, similarly as in [17], the proof of Theorem 2 follows from a Liouville property on the linearized equation around $Q$, see Theorem 3 in Section 3.

With respect to the gKdV case, there are two main difficulties:

(1) $L^{2}$ monotonicity type results, which are similar to the ones for the gKdV equations ([17]), but whose proof are more subtle due to the nonlocal nature of the (BO) operator (see Section 2). For this part, we use a Kato type identity for (1.1) (see [9] and [25]).

(2) The proof of the linear Liouville theorem, which requires the analysis of some linear operators related to $Q$. Note that for this part, we use the fact that $Q(x)$ is explicit, and some known results about the linearized equation 
around $Q$ ([3], [31]). We point out that except for this part of the analysis, all the arguments are quite flexible and could be applied to generalized versions of the (BO) equation. In particular, we do not use the integrability property of the equation.

As a corollary of the proof of Theorem 1 and of Theorem 2, we obtain stability and asymptotic stability of multisoliton solutions. See Theorem 4 in Section 5 for a precise statement.

After the paper was finished and submitted, we learned that S. Gustafson, H. Takaoka, and T-P. Tsai [10] have obtained independently the stability part of Theorem 4. Note that the main result of the present paper, i.e. asymptotic stability of (single or multi-) solitons is not addressed in [10].

The rest of the paper is organized as follows. In Section 2, we prove $L^{2}$ monotonicity type results in the context of Theorem 1. In Section 3, we state and prove the linear Liouville Theorem, which is the main ingredient of the proof of Theorem 2. In Section 4, we prove Theorems 1 and 2 using Sections 2 and 3. Section 5 is devoted to the multisoliton case. In Section 6, we prove some weak convergence and well-posedness results used in the proofs. Finally, Appendix A contains the proof of some technical points.

\section{Monotonicity arguments}

\subsection{Modulation}

Lemma 1 (Choice of translation parameter). There exist $C, \alpha_{0}>0$ such that for any $0<\alpha<\alpha_{0}$, if $u(t)$ is an $H^{\frac{1}{2}}$ solution of (1.1) such that

$$
\forall t \in \mathbb{R}, \quad \inf _{r \in \mathbb{R}}\|u(t)-Q(.-r)\|_{H^{\frac{1}{2}}}<\alpha,
$$

then there exists $\rho(t) \in C^{1}(\mathbb{R})$ such that

$$
\eta(t, x)=u(t, x+\rho(t))-Q(x)
$$

satisfies

$$
\begin{aligned}
\forall t \in \mathbb{R}, \quad & \int Q^{\prime}(x) \eta(t, x) d x=0, \quad\|\eta(t)\|_{H^{\frac{1}{2}}} \leq C \alpha \\
& \left|\rho^{\prime}(t)-1\right| \leq C\left(\int \frac{\eta^{2}(t, x)}{1+x^{2}} d x\right)^{\frac{1}{2}} \leq C\|\eta(t)\|_{L^{2}}
\end{aligned}
$$

Proof of Lemma 1. This follows from standard arguments (see e.g. [4], Lemma 4.1, [15], Proposition 1 and Lemma 4). 
Time independent arguments. For $u \in H^{\frac{1}{2}}$ and $y \in \mathbb{R}$, set

$I_{y}(u)=\int Q^{\prime}(x)(u(x+y)-Q(x)) d x \quad$ so that $\left.\quad \frac{\partial I_{y}}{\partial y}\right|_{y=0, u=Q}=\int\left(Q^{\prime}\right)^{2}>0$.

Thus, by the implicit function theorem, there exists $\alpha_{1}>0, V$ a neighborhood of 0 in $\mathbb{R}$ and a unique $C^{1}$ map:

$$
\begin{aligned}
& y:\left\{u \in H^{\frac{1}{2}},\|u-Q\|_{H^{\frac{1}{2}}} \leq \alpha_{1}\right\} \rightarrow V \\
& \text { such that } I_{y(u)}(u)=0,|y(u)| \leq C\|u-Q\|_{H^{\frac{1}{2}}} .
\end{aligned}
$$

We uniquely extend the $C^{1} \operatorname{map} y(u)$ to $U_{\alpha_{1}}=\left\{u \in H^{\frac{1}{2}}, \inf _{r} \| u(.+r)-\right.$ $\left.Q \|_{H^{\frac{1}{2}}} \leq \alpha_{1}\right\}$ so that for all $u$ and $r, y(u)=y(u(.+r))+r$. Then, we set $\eta_{u}(x)=u(x+y(u))-Q(x)$, so that

$$
\int \eta_{u} Q^{\prime}=0 \quad \text { and } \quad\left\|\eta_{u}\right\|_{H^{\frac{1}{2}}} \leq C\|u-Q\|_{H^{\frac{1}{2}}} .
$$

Estimates depending on $t$. For all $t$, we define $\rho(t)=y(u(t))$ and $\eta(t)=\eta_{u(t)}$. To conclude the proof of the lemma, we just have to prove the estimate on $\rho^{\prime}(t)-1$.

We perform formal computations which can be justified for $H^{\frac{1}{2}}$ solutions by density and continuous dependence arguments. The function $\eta(t, x)$ satisfies the following equation:

$$
\eta_{t}=\left(\mathcal{L} \eta-\frac{1}{2} \eta^{2}\right)_{x}+\left(\rho^{\prime}-1\right)(Q+\eta)_{x} \quad \text { where } \mathcal{L} \eta=-\mathcal{H} \eta_{x}+\eta-Q \eta
$$

Thus, multiplying the equation of $\eta$ by $Q^{\prime}$ and using $\int \eta Q^{\prime}=0$, we obtain

$$
\left(\rho^{\prime}-1\right)\left[\int\left(Q^{\prime}\right)^{2}-\int \eta Q^{\prime \prime}\right]=\int \eta \mathcal{L}\left(Q^{\prime \prime}\right)-\frac{1}{2} \int \eta^{2} Q^{\prime \prime}
$$

which finishes the proof for $\alpha_{0}$ small enough.

Remark 2. By the proof of Lemma 1, $\rho(t)$ depends continuously on $u(t)$ in $H^{\frac{1}{2}}$. In particular, let $u(t)$ satisfy the assumptions of Lemma 1 with $u(0)=u_{0}$. If $u_{n}(0) \rightarrow u_{0}$ in $H^{\frac{1}{2}}$ as $n \rightarrow+\infty$, then by continuous dependence (see [11]), we obtain for all $t \in \mathbb{R}, \rho_{n}(t) \rightarrow \rho(t)$ as $n \rightarrow+\infty$, where $\rho_{n}(t)$ is defined from $u_{n}(t)\left(u_{n}(t)\right.$ is the solution of (1.1) corresponding to $\left.u_{n}(0)=u_{0 n}\right)$.

Note also that in the proof of Lemma 1, we can replace the space $H^{\frac{1}{2}}$ by $L^{2}$, so that in the same context if $u_{n}(0) \rightarrow u_{0}$ in $L^{2}$ as $n \rightarrow+\infty$ then for all $t \in \mathbb{R}, \rho_{n}(t) \rightarrow \rho(t)$ as $n \rightarrow+\infty$ (see continuous dependence in $L^{2}$ also in $[11])$. 
Finally, for future reference, we justify that if $u_{n} \rightarrow u$ in $H^{\frac{1}{2}}$ weak, then $y\left(u_{n}\right) \rightarrow y(u)$, where $y(u)$ is defined in the proof of Lemma 1. Indeed, in this proof, by the decay of $Q^{\prime}(x)$, we can also replace $H^{\frac{1}{2}}$ by the weighted space $L^{2}\left(\frac{1}{1+|x|} d x\right)$, so that if $u_{n} \rightarrow u$ in $L_{l o c}^{2}$ and $\left\|u_{n}\right\|_{L^{2}}+\|u\|_{L^{2}} \leq C$, then $y\left(u_{n}\right) \rightarrow y(u)$ as $n \rightarrow+\infty$.

In the rest of this section, we present monotonicity arguments on $L^{2}$ quantities for both $u(t)$ and $\eta(t)$, in the context of Lemma 1 . These results are reminiscent of similar results for the gKdV equation in [17] and [20], but due to the nonlocal nature of the operator $\mathcal{H}$, the proofs are more involved.

\subsection{Monotonicity results for $u(t)$}

Let $A>1$ to be chosen later and set

$$
\varphi(x)=\varphi_{A}(x)=\frac{\pi}{2}+\arctan \left(\frac{x}{A}\right) \quad \text { so that } \quad \varphi^{\prime}(x)=\frac{\frac{1}{A}}{1+\left(\frac{x}{A}\right)^{2}}>0 .
$$

Proposition 1. Let $0<\lambda<1$. Under the assumptions of Lemma 1, for $\alpha_{0}$ small enough and $A$ large enough, there exists $C>0$ such that for all $x_{0}>1, t_{1} \leq t_{2}$

1. Monotonicity on the right of the soliton:

$$
\begin{aligned}
& \int u^{2}\left(t_{2}, x\right) \varphi\left(x-\rho\left(t_{2}\right)-x_{0}\right) d x \\
& \quad \leq \int u^{2}\left(t_{1}, x\right) \varphi\left(x-\rho\left(t_{1}\right)-\lambda\left(t_{2}-t_{1}\right)-x_{0}\right) d x+\frac{C}{x_{0}} .
\end{aligned}
$$

2. Monotonicity on the left of the soliton:

$$
\begin{gathered}
\int u^{2}\left(t_{2}, x\right) \varphi\left(x-\rho\left(t_{2}\right)+\lambda\left(t_{2}-t_{1}\right)+x_{0}\right) d x \\
\leq \int u^{2}\left(t_{1}, x\right) \varphi\left(x-\rho\left(t_{1}\right)+x_{0}\right) d x+\frac{C}{x_{0}} .
\end{gathered}
$$

Proof of Proposition 1. First, we note that (2.8) is a consequence of $(2.7)$ and the $L^{2}$ norm conservation. Indeed, let $v(t, x)=u(-t,-x)$. Then $v(t)$ is a solution of (1.1) satisfying the assumptions of Lemma 1 and $\rho_{v}(t)=-\rho(-t)$. Thus, from (2.7) applied on $v(t, x)$, we deduce

$$
\begin{aligned}
& \int u^{2}\left(-t_{2}, x\right) \varphi\left(-x+\rho\left(-t_{2}\right)-x_{0}\right) d x \\
& \quad \leq \int u^{2}\left(-t_{1}, x\right) \varphi\left(-x+\rho\left(-t_{1}\right)-\lambda\left(t_{2}-t_{1}\right)-x_{0}\right) d x+\frac{C}{x_{0}} .
\end{aligned}
$$


Since $\varphi(x)=\pi-\varphi(-x)$, from $\int u^{2}\left(-t_{2}\right)=\int u^{2}\left(-t_{1}\right)$, we obtain

$$
\begin{aligned}
& \int u^{2}\left(-t_{2}, x\right) \varphi(x\left.-\rho\left(-t_{2}\right)+x_{0}\right) d x+\frac{C}{x_{0}} \\
& \geq \int u^{2}\left(-t_{1}, x\right) \varphi\left(x-\rho\left(-t_{1}\right)+\lambda\left(t_{2}-t_{1}\right)+x_{0}\right) d x
\end{aligned}
$$

which is exactly formula (2.8) for $t_{2}^{\prime}=-t_{1}, t_{1}^{\prime}=-t_{2}$.

We are reduced to prove (2.7). We perform calculations on regular solutions and then use density arguments and continuous dependence to obtain the result in the framework of Lemma 1.

First, we recall a Kato type identity for solutions of the BO equation. By direct computations, we have

$$
\begin{aligned}
\frac{1}{2} \frac{d}{d t} \int u^{2}(t, x) \varphi(x) d x & =\int u_{t} u \varphi(x) d x=-\int\left(\mathcal{H} u_{x x}+u u_{x}\right) u \varphi(x) d x \\
& =\int\left(\mathcal{H} u_{x}\right)\left(u \varphi^{\prime}(x)+u_{x} \varphi(x)\right) d x+\frac{1}{3} \int u^{3} \varphi^{\prime}(x) d x .
\end{aligned}
$$

For the first term in (2.11), we prove the following result.

Lemma 2. For all $u \in H^{1}(\mathbb{R})$,

$$
\int\left(\mathcal{H} u_{x}\right) u \varphi^{\prime}(x) d x \leq \frac{C}{A} \int u^{2} \varphi^{\prime}(x) d x
$$

Proof of Lemma 2. For $f \in L^{2}(\mathbb{R})$, we define the harmonic extension of $f$ on $\mathbb{R} \times \mathbb{R}_{+}=\mathbb{R}_{+}^{2}$,

$$
\forall x \in \mathbb{R}, \quad F(x, 0)=f(x)
$$

$$
\text { and } F(x, y)=\frac{1}{\pi} \int_{-\infty}^{\infty} \frac{y}{\left(x-x^{\prime}\right)^{2}+y^{2}} f\left(x^{\prime}\right) d x^{\prime}, \quad \text { if } y>0 .
$$

In particular, recall that $\mathcal{H} f^{\prime}(x)=\partial_{y} F(x, 0)$ (see Stein [26, Chapter III], and the Introduction of Toland [28]).

We denote by $\Phi(x, y)$ the harmonic extension of $\varphi^{\prime}(x)$ and $U(x, y)$ the harmonic extension of $u(x)$ on $\mathbb{R} \times \mathbb{R}_{+}$. Note that $\Phi(x, y)$ is explicitly given by

$$
\Phi(x, y)=\frac{1}{A} \frac{1+\frac{y}{A}}{\left(\frac{x}{A}\right)^{2}+\left(1+\frac{y}{A}\right)^{2}} .
$$


Then, by the Green Formula on $\mathbb{R}_{+}^{2}$ (using decay properties of $\Phi(x, y)$ and $\left.\Delta U^{2}=2|\nabla U|^{2}\right)$, we obtain formally

$$
\begin{aligned}
\int\left(\mathcal{H} u_{x}\right) u \varphi^{\prime} & =\int \partial_{y} U(t, x, 0) U(t, x, 0) \Phi(x, 0) d x=\frac{1}{2} \int_{y=0} \partial_{y}\left(U^{2}\right) \Phi d x \\
& =-\frac{1}{2} \iint_{\mathbb{R}_{+}^{2}}\left(\Delta U^{2}\right) \Phi+\frac{1}{2} \iint_{\mathbb{R}_{+}^{2}} U^{2} \Delta \Phi+\frac{1}{2} \int_{y=0} U^{2} \partial_{y} \Phi \\
& =-\iint_{\mathbb{R}_{+}^{2}}|\nabla U|^{2} \Phi+\frac{1}{2} \int u^{2}\left(\mathcal{H} \varphi^{\prime \prime}\right) d x .
\end{aligned}
$$

See Appendix A.1 for a rigorous proof of (2.15). Since $\Phi \geq 0$ on $\mathbb{R}_{+}^{2}$, we obtain

$$
\int\left(\mathcal{H} u_{x}\right) u \varphi^{\prime} \leq \frac{1}{2} \int u^{2}\left(\mathcal{H} \varphi^{\prime \prime}\right)
$$

By explicit computations, since $\mathcal{H}\left(\frac{1}{1+x^{2}}\right)=-\frac{x}{1+x^{2}}$, we have

$$
\mathcal{H} \varphi^{\prime}=-\frac{1}{A^{2}} \frac{x}{1+\left(\frac{x}{A}\right)^{2}}, \quad \mathcal{H} \varphi^{\prime \prime}=\frac{1}{A} \varphi^{\prime}-2\left(\varphi^{\prime}\right)^{2} \quad \text { and } \quad \mathcal{H} \varphi^{\prime \prime} \leq \frac{1}{A} \varphi^{\prime}
$$

Lemma 2 follows.

For the second term in (2.11), we have the following.

Lemma 3. For all $u \in H^{1}(\mathbb{R})$,

$$
\left|\int\left(\mathcal{H} u_{x}\right) u_{x} \varphi d x\right| \leq \frac{C}{A} \int u^{2} \varphi^{\prime}(x) d x
$$

Proof of Lemma 3. We prove (2.18) for $u$ smooth and compactly supported in $\mathbb{R}$, the general case will follow by a density argument.

Since the limit in (1.2) holds in $L^{2}$ (see Stein [26, Chapter II]), we have

$$
\begin{aligned}
& \int\left(\mathcal{H} u_{x}\right) u_{x} \varphi d x=\frac{1}{\pi} \int \text { p.v. }\left(\int \frac{u_{x}(y)}{y-x} d y\right) u_{x}(x) \varphi(x) d x \\
& \quad=\frac{1}{\pi} \lim _{\varepsilon \rightarrow 0} \iint_{|y-x|>\varepsilon} u_{x}(y) u_{x}(x) \frac{\varphi(x)}{y-x} d y d x \\
& \quad=\frac{1}{2 \pi} \iint u_{x}(y) u_{x}(x) \frac{\varphi(x)-\varphi(y)}{y-x} d x d y=\frac{1}{2 \pi} \iint u(y) u(x) K_{\varphi}(x, y) d x d y,
\end{aligned}
$$

by symmetry and then integration by parts, where

$$
\begin{aligned}
K_{\varphi}(x, y) & =-\frac{\partial^{2}}{\partial x \partial y}\left(\frac{\varphi(x)-\varphi(y)}{x-y}\right) \\
& =\frac{2(\varphi(x)-\varphi(y))-\left(\varphi^{\prime}(x)+\varphi^{\prime}(y)\right)(x-y)}{(x-y)^{3}}
\end{aligned}
$$


Note that all the integrals in (2.19) make sense since $u(x)$ is compactly supported, $(\varphi(x)-\varphi(y)) /(x-y)$ is bounded and moreover, by subtracting the following two Taylor formulas:

$$
\begin{aligned}
& \varphi(x)=\varphi(y)+(x-y) \varphi^{\prime}(y)+\frac{1}{2}(x-y)^{2} \varphi^{\prime \prime}(y)+\frac{1}{6}(x-y)^{3} \varphi^{\prime \prime \prime}\left(x_{1}\right), \\
& \varphi(y)=\varphi(x)+(y-x) \varphi^{\prime}(x)+\frac{1}{2}(y-x)^{2} \varphi^{\prime \prime}(x)+\frac{1}{6}(y-x)^{3} \varphi^{\prime \prime \prime}\left(x_{2}\right),
\end{aligned}
$$

where $x_{1}, x_{2} \in(y, x)$, we find:

$$
K_{\varphi}(x, y)=\frac{1}{2} \frac{\varphi^{\prime \prime}(y)-\varphi^{\prime \prime}(x)}{x-y}+\frac{1}{6}\left(\varphi^{\prime \prime \prime}\left(x_{1}\right)+\varphi^{\prime \prime \prime}\left(x_{2}\right)\right),
$$

which is also bounded on $\mathbb{R}^{2}$. Note also that by explicit computations, we have

$$
\varphi^{\prime \prime \prime}(x)=\frac{\varphi^{\prime}(x)}{A^{2}}\left(\frac{-2}{1+\left(\frac{x}{A}\right)^{2}}+\frac{8\left(\frac{x}{A}\right)^{2}}{\left(1+\left(\frac{x}{A}\right)^{2}\right)^{2}}\right)=\frac{\varphi^{\prime}(x)}{A}\left(-2 \varphi^{\prime}(x)+\frac{8}{A} x^{2}\left(\varphi^{\prime}\right)^{2}\right) .
$$

We are reduced to prove the following estimate

$$
\left|\iint u(y) u(x) K_{\varphi}(x, y) d x d y\right| \leq \frac{C}{A} \int u^{2} \varphi^{\prime}(x) d x .
$$

We consider only the case $|y|<|x|$ (by symmetry), and we divide $\{(x, y)$ : $|y|<|x|\}$ into the following regions:

- $\Sigma_{1}=\left\{(x, y): x>A, 0<y<\frac{x}{2}\right\}$. For $(x, y) \in \Sigma_{1}$, by (2.20) and the fact that $\varphi^{\prime}$ is decreasing on $\mathbb{R}^{+}$, we have

$$
\left|K_{\varphi}(x, y)\right| \leq \frac{4}{(x-y)^{2}} \sup _{[y, x]} \varphi^{\prime} \leq \frac{16}{x^{2}} \varphi^{\prime}(y)=\frac{16}{A^{2}} \frac{1}{\left(\frac{x}{A}\right)^{2}} \varphi^{\prime}(y) \leq \frac{32}{A} \varphi^{\prime}(x) \varphi^{\prime}(y) .
$$

Thus, by Cauchy-Schwarz inequality, since $\int \varphi^{\prime}(x)=\pi$, we obtain

$$
\begin{aligned}
\left|\iint_{\Sigma_{1}} u(y) u(x) K_{\varphi}(x, y) d x d y\right| & \leq \frac{C}{A} \int|u(x)| \varphi^{\prime}(x) d x \int|u(y)| \varphi^{\prime}(y) d y \\
& \leq \frac{C \pi}{A} \int u^{2}(x) \varphi^{\prime}(x) d x .
\end{aligned}
$$

The case of the region $\Sigma_{1}^{-}=\left\{(x, y): x<-A, \frac{x}{2}<y<0\right\}$ is similar.

- $\Sigma_{2}=\{(x, y): x>A,-x<y<0\}$. For $(x, y) \in \Sigma_{2}$, we have by $(2.20)$, $|x-y|=x-y>x>\frac{1}{2}(x+A), \varphi^{\prime}(y)>\varphi^{\prime}(x)$ and so by (2.20) and $\varphi$ bounded, we obtain

$$
\left|K_{\varphi}(x, y)\right| \leq \frac{C}{(x+A)^{3}}+\frac{C \varphi^{\prime}(y)}{x^{2}} .
$$


For the term $\frac{C \varphi^{\prime}(y)}{x^{2}}$, we argue as for $\Sigma_{1}$. For the other term, by CauchySchwarz' inequality and the expression of $\varphi^{\prime}$, we have

$$
\begin{aligned}
& \iint_{\Sigma_{2}}|u(y)||u(x)| \frac{1}{(x+A)^{3}} d x d y \\
& \leq C\left(\iint_{\Sigma_{2}} \frac{u^{2}(x)}{(x+A)^{3}} d x d y\right)^{\frac{1}{2}}\left(\iint_{\Sigma_{2}} \frac{u^{2}(y)}{(x+A)^{3}} d x d y\right)^{\frac{1}{2}} \\
& \leq C\left(\frac{1}{2} \int_{x>A} \frac{u^{2}(x)}{(x+A)^{2}} d x\right)^{\frac{1}{2}}\left(\frac{1}{2} \int_{y<0} \frac{u^{2}(y)}{(-y+A)^{2}} d y\right)^{\frac{1}{2}} \leq \frac{C^{\prime}}{A} \int u^{2}(x) \varphi^{\prime}(x) d x .
\end{aligned}
$$

The case of $\Sigma_{2}^{-}=\{(x, y): x<-A, 0<y<-x\}$ is similar to $\Sigma_{2}$.

- $\Sigma_{3}=\{(x, y):|x|<A,|y|<|x|\}$. For $(x, y) \in \Sigma_{3}$, and $|s|<|x|$, we have $\frac{1}{2 A} \leq \varphi^{\prime}(s) \leq \frac{1}{A}$ and thus, from (2.21) and (2.22), we obtain

$$
\left|K_{\varphi}(x, y)\right| \leq C \sup _{|s|<|x|}\left|\varphi^{\prime \prime \prime}(s)\right| \leq \frac{C}{A^{3}} \leq \frac{C}{A} \varphi^{\prime}(x) \varphi^{\prime}(y) .
$$

We finish as for $\Sigma_{1}$.

- $\Sigma_{4}=\left\{(x, y): x>A, \frac{1}{2} x<y<x\right\}$. For $(x, y) \in \Sigma_{4}$, and $y<s<x$, we have from $(2.22)$ :

$$
\left|\varphi^{\prime \prime \prime}(s)\right| \leq \frac{10}{A}\left(\varphi^{\prime}(s)\right)^{2} \leq \frac{10}{A} \varphi^{\prime}(y) \varphi^{\prime}(s) \leq \frac{40}{A} \varphi^{\prime}(y) \varphi^{\prime}(x)
$$

thus

$$
\left|K_{\varphi}(x, y)\right| \leq \frac{C}{A} \varphi^{\prime}(x) \varphi^{\prime}(y),
$$

and we conclude as for $\Sigma_{1}$. The case of $\Sigma_{4}^{-}=\left\{(x, y): x<-A, x<y<\frac{x}{2}\right\}$ is similar

In conclusion, we have obtained (2.23) and Lemma 3 is proved.

From (2.11), Lemmas 2 and 3, there exists $C_{0}>0$ such that

$$
\frac{1}{2} \frac{d}{d t} \int u^{2}(t, x) \varphi(x) d x \leq \frac{C_{0}}{A} \int u^{2}(t, x) \varphi^{\prime}(x) d x+\frac{1}{3} \int\left|u^{3}(t, x)\right| \varphi^{\prime}(x) d x .
$$

Now, let $u(t)$ be a solution of (1.1) satisfying the assumptions of Lemma 1 on $\mathbb{R}$. Let $\eta(t), \rho(t)$ be associated to the decomposition of $u(t)$ on $I$ as in Lemma 1 .

Let $0<\lambda<1, t_{0} \in\left[t_{1}, t_{2}\right]$ and $x_{0} \geq 1$. For any $t \in\left[t_{1}, t_{0}\right], x \in \mathbb{R}$, we set

$$
\widetilde{x}=x-x_{0}-\rho(t)-\lambda\left(t_{0}-t\right), \quad M_{\varphi}(t)=\frac{1}{2} \int u^{2}(t, x) \varphi(\widetilde{x}) d x .
$$


Then, by (2.24), we find

$$
M_{\varphi}^{\prime}(t) \leq-\frac{1}{2}\left(\rho^{\prime}(t)-\lambda-\frac{2 C_{0}}{A}\right) \int u^{2}(t) \varphi^{\prime}(\widetilde{x})+\frac{1}{3} \int|u(t)|^{3} \varphi^{\prime}(\widetilde{x}) .
$$

Fix now $A>0$ large enough so that $\frac{2 C_{0}}{A} \leq \frac{1}{4}(1-\lambda)$. Then, by $(2.2)$, we choose $\alpha_{0}>0$ small enough so that $\forall t \in I, \rho^{\prime}(t)-\lambda>\frac{1}{2}(1-\lambda)$. Therefore, we obtain

$$
M_{\varphi}^{\prime}(t) \leq-\frac{1}{8}(1-\lambda) \int u^{2}(t) \varphi^{\prime}(\widetilde{x})+\frac{1}{3} \int|u(t)|^{3} \varphi^{\prime}(\widetilde{x})
$$

Finally, we estimate the nonlinear term $\int|u(t)|^{3} \varphi^{\prime}(\widetilde{x})$. We first observe:

$$
\int|u(t)|^{3} \varphi^{\prime}(\widetilde{x}) \leq C \int Q^{3}(x-\rho(t)) \varphi^{\prime}(\widetilde{x}) d x+C \int|\eta(t, x)|^{3} \varphi^{\prime}(\widetilde{x}) d x .
$$

For the first term, we distinguish two regions in $x$ :

- $\Omega_{1}=\left\{x: x<\rho(t)+\frac{1}{2} x_{0}+\frac{1}{2} \lambda\left(t_{0}-t\right)\right\}$. For $x \in \Omega_{1}$, we have $\widetilde{x}<-\frac{1}{2} x_{0}-\frac{1}{2} \lambda\left(t_{0}-t\right)$, and thus

$$
\varphi^{\prime}(\widetilde{x}) \leq \frac{C}{\left(x_{0}+\lambda\left(t_{0}-t\right)\right)^{2}}
$$

This implies

$$
\int_{\Omega_{1}} Q^{3}(x-\rho(t)) \varphi^{\prime}(\widetilde{x}) \leq \frac{C}{\left(x_{0}+\lambda\left(t_{0}-t\right)\right)^{2}} \int Q^{3} \leq \frac{C}{\left(x_{0}+\lambda\left(t_{0}-t\right)\right)^{2}}
$$

- $\Omega_{2}=\left\{x>\rho(t)+\frac{1}{2} x_{0}+\frac{1}{2} \lambda\left(t_{0}-t\right)\right\}$. For $x \in \Omega_{2}$, we have $x-\rho(t)>$ $\frac{1}{2} x_{0}+\frac{1}{2} \lambda\left(t_{0}-t\right)$ and thus $Q^{3}(x-\rho(t)) \leq \frac{C}{\left(x_{0}+\lambda\left(t_{0}-t\right)\right)^{6}}$, and

$$
\int_{\Omega_{2}} Q^{3}(x-\rho(t)) \varphi^{\prime}(\widetilde{x}) d x \leq \frac{C}{\left(x_{0}+\lambda\left(t_{0}-t\right)\right)^{6}} .
$$

Now, we claim

$$
\int|\eta(t, x-\rho(t))|^{3} \varphi^{\prime}(\widetilde{x}) d x \leq C \alpha_{0} \int \eta^{2}(t, x-\rho(t)) \varphi^{\prime}(\widetilde{x}) d x
$$

where $C$ is independent of $A$. See proof of (2.30) in Appendix A.2. Moreover, as before, we find

$$
\begin{aligned}
\int \eta^{2}(t, x-\rho(t)) \varphi^{\prime}(\widetilde{x}) d x & \leq C \int\left(u^{2}(t, x)+Q^{2}(x-\rho(t))\right) \varphi^{\prime}(\widetilde{x}) d x \\
& \leq C \int u^{2}(t, x) \varphi^{\prime}(\widetilde{x}) d x+\frac{C}{\left(x_{0}+\lambda\left(t_{0}-t\right)\right)^{2}} .
\end{aligned}
$$


Thus, it follows from (2.27)-(2.30) that for $\alpha_{0}>0$ small enough, $\forall t \in\left[t_{1}, t_{0}\right]$,

$$
\begin{aligned}
& M_{\varphi}^{\prime}(t) \leq-\frac{1}{8}(1-\lambda) \int u^{2}(t) \varphi^{\prime}(\widetilde{x})+C \alpha_{0} \int u^{2}(t) \varphi^{\prime}(\widetilde{x})+\frac{C}{\left(x_{0}+\lambda\left(t_{0}-t\right)\right)^{2}} \\
& \quad \leq-\frac{1}{16}(1-\lambda) \int u^{2}(t) \varphi^{\prime}(\widetilde{x})+\frac{C}{\left(x_{0}+\lambda\left(t_{0}-t\right)\right)^{2}} .
\end{aligned}
$$

Let $t \in\left[t_{1}, t_{0}\right]$. By integration of $(2.32)$ on $\left[t, t_{0}\right]$, since

$$
\int_{t}^{t_{0}} \frac{d t^{\prime}}{\left(x_{0}+\lambda\left(t_{0}-t^{\prime}\right)\right)^{2}}=\frac{1}{\lambda x_{0}} \int_{0}^{\frac{\lambda\left(t_{0}-t\right)}{x_{0}}} \frac{d t^{\prime \prime}}{\left(1+t^{\prime \prime}\right)^{2}} \leq \frac{C}{x_{0}}, \quad\left(t^{\prime \prime}=\frac{\lambda}{x_{0}}\left(t_{0}-t^{\prime}\right)\right)
$$

we find:

$$
\begin{aligned}
& \int u^{2}\left(t_{0}, x\right) \varphi\left(x-x_{0}-\rho\left(t_{0}\right)\right) d x+\frac{1}{C} \int_{t}^{t_{0}} \int u^{2}\left(t^{\prime}, x\right) \varphi^{\prime}\left(x-x_{0}-\rho\left(t^{\prime}\right)-\lambda\left(t-t^{\prime}\right)\right) d x d t^{\prime} \\
& (2.33) \leq \int u^{2}(t, x) \varphi\left(x-x_{0}-\rho(t)-\lambda\left(t_{0}-t\right)\right) d x+\frac{C}{x_{0}} .
\end{aligned}
$$

By density and continuous dependence ([11]) estimate (2.33) also holds for $H^{\frac{1}{2}}$ solutions.

\subsection{Monotonicity results for $\eta(t)$}

Here, we present similar monotonicity arguments for $\eta(t)$. See [20] for similar results in the case of the $\mathrm{gKdV}$ equations.

Proposition 2. Let $0<\lambda<1$. Under the assumptions of Lemma 1, for $\alpha_{0}$ small enough and $A$ large enough, there exists $C>0$ such that for all $x_{0}>1$, $t_{1} \leq t_{2}$

$$
\begin{aligned}
& \int \eta^{2}\left(t_{2}, x\right)\left(\varphi\left(x-x_{0}\right)-\varphi\left(-x_{0}\right)\right) d x \\
& \leq \int \eta^{2}\left(t_{1}, x\right)\left(\varphi\left(x-\lambda\left(t_{2}-t_{1}\right)-x_{0}\right)-\varphi\left(-x_{0}-\lambda\left(t_{2}-t_{1}\right)\right)\right) d x \\
& \quad+C \int_{t_{1}}^{t_{2}} \frac{\|\eta(t)\|_{L^{2}}^{2}}{\left(x_{0}+\lambda\left(t_{2}-t\right)\right)^{2}} d t
\end{aligned}
$$

Remark 3. With respect to Proposition 1, we need to modify slighty the function in the integral $\left(\varphi\left(x-x_{0}\right)-\varphi\left(-x_{0}\right)\right.$ instead of $\left.\varphi\left(x-x_{0}\right)\right)$ to remove some terms in the second member, see comments in the proof. This estimate is clearly improving Proposition 1 since the remainder term can now be controlled by $\frac{C}{x_{0}} \sup _{t}\|\eta(t)\|_{L^{2}}^{2}$. 
As for $u(t)$ in the proof of Proposition 1, we have by direct computations using (2.4),

$$
\begin{aligned}
\frac{1}{2} & \frac{d}{d t} \int \eta^{2}(t, x) \varphi(x) d x=\int \eta_{t} \eta \varphi(x) d x \\
= & -\int(\mathcal{L} \eta)\left(\eta \varphi^{\prime}+\eta_{x} \varphi\right)+\frac{1}{3} \int \eta^{3} \varphi^{\prime}+\left(\rho^{\prime}-1\right)\left(\int Q^{\prime} \eta \varphi-\frac{1}{2} \int \eta^{2} \varphi^{\prime}\right) \\
& =\int\left(\mathcal{H} \eta_{x}\right) \eta \varphi^{\prime}+\int\left(\mathcal{H} \eta_{x}\right) \eta_{x} \varphi-\frac{1}{2} \int \eta^{2} \varphi^{\prime}+\frac{1}{2} \int \eta^{2}\left(Q \varphi^{\prime}-Q^{\prime} \varphi\right)+\frac{1}{3} \int \eta^{3} \varphi^{\prime} \\
& +\left(\rho^{\prime}-1\right)\left(\int Q^{\prime} \eta \varphi-\frac{1}{2} \int \eta^{2} \varphi^{\prime}\right) .
\end{aligned}
$$

Let $0<\lambda<1$ and $\bar{x}=x-x_{0}-\lambda\left(t_{0}-t\right)$. Then, by Lemmas 2 and 3 , we get

$$
\begin{aligned}
\frac{d}{d t} \int \eta^{2} \varphi(\bar{x}) \leq- & \left(\rho^{\prime}(t)-\lambda-\frac{2 C_{0}}{A}\right) \int \eta^{2} \varphi^{\prime}(\bar{x})+\int \eta^{2}\left(Q \varphi^{\prime}(\bar{x})-Q^{\prime} \varphi(\bar{x})\right) \\
& +\frac{2}{3} \int|\eta|^{3} \varphi^{\prime}(\bar{x})+2\left(\rho^{\prime}-1\right) \int Q^{\prime} \eta \varphi(\bar{x}) .
\end{aligned}
$$

Now, as in the proof of Proposition 1, we fix $A>1$ such that $\frac{2 C_{0}}{A} \leq \frac{1}{4}(1-\lambda)$ and $\alpha_{0}$ small enough so that $\rho^{\prime}-\lambda>\frac{1}{2}(1-\lambda)$ by (2.2). Then, by $(2.30)$ and (2.2), we can choose $\alpha_{0}>0$ small enough so that

$$
\frac{2}{3} \int|\eta|^{3} \varphi^{\prime}(\bar{x}) \leq \frac{1}{8}(1-\lambda) \int \eta^{2} \varphi^{\prime}(\bar{x})
$$

Thus, we obtain

$\frac{d}{d t} \int \eta^{2} \varphi(\bar{x}) \leq \frac{-1}{8}(1-\lambda) \int \eta^{2} \varphi^{\prime}(\bar{x})+\int \eta^{2}\left(Q \varphi^{\prime}(\bar{x})-Q^{\prime} \varphi(\bar{x})\right)+2\left(\rho^{\prime}-1\right) \int Q^{\prime} \eta \varphi(\bar{x})$.

At this point, note that the term $\int \eta^{2} Q^{\prime} \varphi(\bar{x})$ has no sign, and since $\varphi(y) \sim \frac{C}{|y|}$ as $y \rightarrow-\infty$, this term can only be controlled by $\frac{C}{\left(x_{0}+\lambda\left(t_{0}-t\right)\right)} \int \eta^{2}$, which is not sufficient for our purposes. We modify slightly the functional to cancel the main order of this term.

Indeed, since $\int \eta Q^{\prime}=0$, using (2.4), we have

$$
\frac{d}{d t} \int \eta^{2}=2 \int Q \eta \eta_{x}=-\int Q^{\prime} \eta^{2}
$$


Therefore, using also $\int Q^{\prime} \eta=0$, we get

$$
\begin{aligned}
& \frac{d}{d t} \int \eta^{2}\left(\varphi(\bar{x})-\varphi\left(-x_{0}-\lambda\left(t_{0}-t\right)\right)\right) \leq-\frac{1}{8}(1-\lambda) \int \eta^{2} \varphi^{\prime}(\bar{x}) \\
& +\int \eta^{2}\left(Q \varphi^{\prime}(\bar{x})-Q^{\prime}\left(\varphi(\bar{x})-\varphi\left(-x_{0}-\lambda\left(t_{0}-t\right)\right)\right)\right) \\
& +2\left(\rho^{\prime}-1\right) \int \eta Q^{\prime}\left(\varphi(\bar{x})-\varphi\left(-x_{0}-\lambda\left(t_{0}-t\right)\right)\right)-\lambda \varphi^{\prime}\left(-\left(x_{0}+\lambda\left(t_{0}-t\right)\right)\right) \int \eta^{2} .
\end{aligned}
$$

Now, we claim the following estimate $\forall x \in \mathbb{R}$,

$$
Q(x) \varphi^{\prime}(\bar{x})+\left|Q(x)\left(\varphi(\bar{x})-\varphi\left(-\left(x_{0}+\lambda\left(t_{0}-t\right)\right)\right)\right)\right| \leq \frac{C}{\left(x_{0}+\lambda\left(t_{0}-t\right)\right)^{2}} .
$$

Since

$$
Q(x) \varphi^{\prime}(\bar{x}) \leq \frac{C}{\left(1+x^{2}\right)\left(1+\left(x-x_{0}-\lambda\left(t_{0}-t\right)\right)^{2}\right)}
$$

(recall that the value of $A$ has been fixed) estimate (2.35) is clear for $Q(x) \varphi^{\prime}(\bar{x})$ by considering the two regions $|x|>\frac{1}{2}\left(x_{0}+\lambda\left(t_{0}-t\right)\right)$ and $|x|<$ $\frac{1}{2}\left(x_{0}+\lambda\left(t_{0}-t\right)\right)$.

For the other term, we first note that since $|Q(x)| \leq \frac{C}{1+x^{2}}$ and $\varphi$ is bounded, the estimate is clear for $|x|>\frac{1}{2}\left(x_{0}+\lambda\left(t_{0}-t\right)\right)$. For $|x|<\frac{1}{2}\left(x_{0}+\right.$ $\left.\lambda\left(t_{0}-t\right)\right)$, we have

$$
\left|\varphi(\bar{x})-\varphi\left(-x_{0}-\lambda\left(t_{0}-t\right)\right)\right| \leq|x|_{\left[\frac { 1 } { 2 } \left(x_{0}+\lambda\left(t_{0}-t\right), \frac{3}{2}\left(x_{0}+\lambda\left(t_{0}-t\right)\right]\right.\right.} \varphi^{\prime} \leq \frac{C|x|}{\left(x_{0}+\lambda\left(t_{0}-t\right)\right)^{2}} ;
$$

thus, for such $x$, we obtain the following estimate which finishes the proof of (2.35):

$$
\left|Q(x)\left(\varphi(\bar{x})-\varphi\left(-x_{0}-\lambda\left(t_{0}-t\right)\right)\right)\right| \leq \frac{C}{\left(x_{0}+\lambda\left(t_{0}-t\right)\right)^{2}}
$$

By (2.2) and (2.35), and since $\left|Q^{\prime}(x)\right| \leq \frac{C}{1+|x|} Q(x)$, we obtain

$$
\left|\int \eta^{2}\left(Q \varphi^{\prime}(\bar{x})-Q^{\prime}\left(\varphi(\bar{x})-\varphi\left(-x_{0}-\lambda\left(t_{0}-t\right)\right)\right)\right)\right| \leq \frac{C\|\eta(t)\|_{L^{2}}^{2}}{\left(x_{0}+\lambda\left(t_{0}-t\right)\right)^{2}}
$$

$$
\begin{aligned}
\left|\left(\rho^{\prime}-1\right) \int Q^{\prime} \eta\left(\varphi(\bar{x})-\varphi\left(-x_{0}-\lambda\left(t_{0}-t\right)\right)\right)\right| & \leq \frac{C\|\eta(t)\|_{L^{2}}}{\left(x_{0}+\lambda\left(t_{0}-t\right)\right)^{2}} \int \frac{|\eta|}{1+|x|} \\
& \leq \frac{C\|\eta(t)\|_{L^{2}}^{2}}{\left(x_{0}+\lambda\left(t_{0}-t\right)\right)^{2}} .
\end{aligned}
$$

The conclusion is thus:

$$
\frac{d}{d t} \int \eta^{2}\left(\varphi(\bar{x})-\varphi\left(-\left(x_{0}+\lambda\left(t_{0}-t\right)\right)\right)\right) \leq-\frac{1}{8}(1-\lambda) \int \eta^{2} \varphi^{\prime}(\bar{x})+\frac{C\|\eta(t)\|_{L^{2}}^{2}}{\left(x_{0}+\lambda\left(t_{0}-t\right)\right)^{2}}
$$


By integration on $\left[t, t_{0}\right]$, we get

$$
\begin{aligned}
\int \eta^{2}\left(t_{0}, x\right)( & \left.\varphi\left(x-x_{0}\right)-\varphi\left(-x_{0}\right)\right) d x \\
& +\frac{1}{C} \int_{t}^{t_{0}} \int \eta^{2}\left(t^{\prime}, x\right) \varphi^{\prime}\left(x-x_{0}-\lambda\left(t_{0}-t^{\prime}\right)\right) d x d t^{\prime} \\
\leq & \int \eta^{2}(t, x)\left(\varphi\left(x-x_{0}-\lambda\left(t_{0}-t\right)\right)-\varphi\left(-x_{0}-\lambda\left(t_{0}-t\right)\right)\right) d x \\
& +C \int_{t}^{t_{0}} \frac{\left\|\eta\left(t^{\prime}\right)\right\|_{L^{2}}^{2} d t^{\prime}}{\left(x_{0}+\lambda\left(t_{0}-t^{\prime}\right)\right)^{2}} .
\end{aligned}
$$

\section{Linear Liouville property}

In this section, we prove the following result.

Theorem 3. Let $w \in C\left(\mathbb{R}, L^{2}(\mathbb{R})\right) \cap L^{\infty}\left(\mathbb{R}, L^{2}(\mathbb{R})\right)$ be a solution of

$$
w_{t}=(\mathcal{L} w)_{x}+\beta(t) Q^{\prime}, \quad(t, x) \in \mathbb{R}^{2}, \quad \text { where } \beta \text { is continuous, }
$$

satisfying

$$
\begin{gathered}
\forall t \in \mathbb{R}, \quad \int w(t, x) Q(x) d x=\int w(t, x) Q^{\prime}(x) d x=0, \\
\forall t \in \mathbb{R}, \forall x_{0}>1, \quad \int_{|x|>x_{0}} w^{2}(t, x) d x \leq \frac{C}{x_{0}} .
\end{gathered}
$$

Then

$$
w \equiv 0 \quad \text { on } \mathbb{R}^{2}
$$

This result is similar to Theorem 3 in [16]. For the proof, we follow the strategy of [14], [19], introducing a dual problem whose operator has better spectral properties. Since $w(t)$ is only $L^{2}$ and has a weak decay at infinity in space, we will need to regularize and localize the dual solution.

For the sake of clarity, we now present the formal argument. The complete justification will be presented in Sections 3.1 and 3.2.

Multiplying the equation of $w(t)$ by $x w(t)$, we get

$$
\frac{d}{d t} \int x w^{2}=-2 \int(\mathcal{H} w) w_{x}-\int w^{2}+\int w^{2}\left(Q-x Q^{\prime}\right)+2 \beta(t) \int x Q^{\prime} w,
$$

where $\left(\int\left(Q^{\prime}\right)^{2}\right) \beta(t)=\int w \mathcal{L}\left(Q^{\prime \prime}\right)$ (multiply the equation of $w$ by $Q^{\prime}$ and use $\left.\int w Q^{\prime}=0\right)$. 
But it is not clear how to study the spectral properties of the operator $2 \int(\mathcal{H} w) w_{x}+\int w^{2}-\int w^{2}\left(Q-x Q^{\prime}\right)+\frac{2}{\int\left(Q^{\prime}\right)^{2}}\left(\int w \mathcal{L} Q^{\prime \prime}\right)\left(\int x Q^{\prime} w\right)$.

Moreover, the decay estimate (3.3) is not quite enough to control $\int x w^{2}$.

Therefore, we instead rely on the dual problem, setting $v=\mathcal{L} w$. Since $\mathcal{L} Q^{\prime}=0$ (direct calculation), we obtain the following equation for $v(t)$ : $v_{t}=\mathcal{L}\left(v_{x}\right)$. Multiplying the equation by $x v$, we obtain

$$
-\frac{d}{d t} \int x v^{2}=2 \int(\mathcal{H} v) v_{x}+\int v^{2}-\int v^{2}\left(Q+x Q^{\prime}\right) .
$$

Note that the operator in $v$ is much easier to study since now the potential $x Q^{\prime}$ has a positive contribution $\left(x Q^{\prime} \leq 0\right)$, moreover, there is no scalar product. In fact, we will obtain (see Proposition 4) the positivity of this operator under the orthogonality condition $\int v(x Q)^{\prime}=0$. Observe that $\int v(x Q)^{\prime}=\int(\mathcal{L} w)(x Q)^{\prime}=-\int w Q=0$ since $\mathcal{L}\left((x Q)^{\prime}\right)=-Q($ see $(3.35))$.

Provided that $\int|x| v^{2}(t) \leq C$, we would obtain from the above identity

$$
\int_{-\infty}^{+\infty}\|v(t)\|_{H^{\frac{1}{2}}}^{2} d t \leq C
$$

which says that for a subsequence $t_{n} \rightarrow+\infty, v\left(t_{n}\right) \rightarrow 0, w\left(t_{n}\right) \rightarrow 0$. Combined with energy conservation $((\mathcal{L} w(t), w(t))=C)$ and Lemma 15 below, this gives $w \equiv 0$. But (3.3) is not enough to obtain the estimate $\int|x| v^{2}(t) \leq C$ In fact, since $w(t)$ is only in $L^{2}$, we both need to localize and regularize the dual problem.

\subsection{Proof of Theorem 3 assuming the positivity of a quadratic form}

Lemma 4. (Regularized dual problem). There exists $\gamma_{0}>0$ such that for any $0<\gamma<\gamma_{0}$, the following is true. Let $v=\left(1-\gamma \partial_{x}^{2}\right)^{-1}(\mathcal{L} w)$. Then, $v \in C\left(\mathbb{R}, H^{1}(\mathbb{R})\right) \cap L^{\infty}\left(\mathbb{R}, H^{1}(\mathbb{R})\right)$ and

1. Equation of $v$.

$$
v_{t}=\mathcal{L}\left(v_{x}\right)-\gamma\left(1-\gamma \partial_{x}^{2}\right)^{-1}\left(2 v_{x x} Q^{\prime}+v_{x} Q^{\prime \prime}\right) .
$$

2. Decay of $v$.

$$
\forall t \in \mathbb{R}, x_{0}>1, \quad \int_{|x|>x_{0}}\left(v_{x}^{2}(t, x)+v^{2}(t, x)\right) d x \leq \frac{C_{\gamma}}{x_{0}^{\frac{3}{4}}} .
$$

3. Virial type estimate.

$$
\int_{-\infty}^{+\infty} \frac{1}{\left(1+t^{2}\right)^{\frac{2}{5}}}\|v(t)\|_{H^{1}}^{2} d t<C
$$


Proof of Lemma 4. First, since $\sup _{t}\|w(t)\|_{L^{2}} \leq C$, we obtain $\sup _{t}\|v(t)\|_{H^{1}}$ $\leq C_{\gamma}$ (see Claim 1 below).

1. Equation of $v$. Let $\widetilde{v}=\mathcal{L} w$ so that $w_{t}=\widetilde{v}_{x}+\beta Q^{\prime}$. Since $\mathcal{L} Q^{\prime}=0$, the function $\widetilde{v}$ satisfies $\widetilde{v}_{t}=\mathcal{L} w_{t}=\mathcal{L}\left(\widetilde{v}_{x}\right)$. Now, we introduce a regularization of the function $\widetilde{v}$. For $0<\gamma<\frac{1}{2}$ to be chosen later small enough, we set:

$$
v(t, x)=\left(1-\gamma \partial_{x}^{2}\right)^{-1} \widetilde{v}(t, x) \quad \text { or equivalently } \quad v-\gamma v_{x x}=\widetilde{v}=\mathcal{L} w
$$

Then, $v(t, x)$ satisfies the following equation

$v_{t}=\left(1-\gamma \partial_{x}^{2}\right)^{-1} \widetilde{v}_{t}=\left(1-\gamma \partial_{x}^{2}\right)^{-1} \mathcal{L}\left(\widetilde{v}_{x}\right)=\mathcal{L}\left(v_{x}\right)-\left(1-\gamma \partial_{x}^{2}\right)^{-1}\left(\widetilde{v}_{x} Q\right)+v_{x} Q$

But $-\left(1-\gamma \partial_{x}^{2}\right)^{-1}\left(\widetilde{v}_{x} Q\right)+v_{x} Q=\left(1-\gamma \partial_{x}^{2}\right)^{-1}\left(-2 \gamma v_{x x} Q^{\prime}-\gamma v_{x} Q^{\prime \prime}\right)$, and so

$$
v_{t}=\mathcal{L}\left(v_{x}\right)-\gamma\left(1-\gamma \partial_{x}^{2}\right)^{-1}\left(2 v_{x x} Q^{\prime}+v_{x} Q^{\prime \prime}\right) .
$$

2. Decay estimate on $v$. By using the decay on $w(t)$, we claim

$$
\forall x_{0}>1, \forall t, \quad \int_{|x| \geq x_{0}}\left(v_{x}^{2}(t, x)+v^{2}(t, x)\right) d x \leq \frac{C_{\gamma}}{x_{0}^{\frac{3}{4}}} .
$$

Indeed, let $\left(x_{0}>1\right)$

$$
h(x)=h_{x_{0}}(x)=\varphi_{\sqrt{x_{0}}}^{2}\left(x-x_{0}\right)=\left(\frac{\pi}{2}+\arctan \left(\frac{x-x_{0}}{\sqrt{x_{0}}}\right)\right)^{2} .
$$

Note that $0 \leq\left|h^{\prime}\right|+\left|h^{\prime \prime}\right| \leq C h$. Since $v-\gamma v_{x x}=\mathcal{L} w$, multiplying by $v h$, we have

$$
\int v^{2} h+\gamma \int v_{x}^{2} h-\frac{1}{2} \gamma \int v^{2} h^{\prime \prime}=\int w \mathcal{L}(v h)=\int w D(v h)+\int w v h-\int Q w v h
$$

First, from

$$
\left|\int w v h\right|+\left|\int Q w v h\right| \leq C\|w \sqrt{h}\|_{L^{2}}\|v \sqrt{h}\|_{L^{2}}
$$

and

$$
\int w^{2} h \leq \int_{x<\frac{x_{0}}{2}} w^{2} h+\int_{x>\frac{x_{0}}{2}} w^{2} \leq C \frac{1}{x_{0}}
$$

(using the definition of $h$ and (3.3)) it follows that

$$
\left|\int w v h\right|+\left|\int Q w v h\right| \leq \frac{C}{x_{0}^{\frac{1}{2}}}\|v \sqrt{h}\|_{L^{2}}
$$


Second, by Lemma 14, we have

$$
\left|\int w D(v h)-\int D(v \sqrt{h}) \sqrt{h} w\right| \leq\|w\|_{L^{2}}\|v \sqrt{h}\|_{L^{4}}\|D(\sqrt{h})\|_{L^{4}} \leq \frac{C}{x_{0}^{\frac{3}{8}}}\|v \sqrt{h}\|_{H^{1}} .
$$

Since

$\left|\int D(v \sqrt{h}) \sqrt{h} w\right| \leq\|w \sqrt{h}\|_{L^{2}}\|v \sqrt{h}\|_{H^{1}}$ and $\|v \sqrt{h}\|_{H^{1}}^{2}=\int\left(v_{x}^{2}+v^{2}\right) h+O\left(\frac{1}{x_{0}}\right)$, we obtain from (3.11)

$$
\int_{x>x_{0}}\left(v_{x}^{2}+v^{2}\right) \leq \int\left(v_{x}^{2}+v^{2}\right) h \leq \frac{C_{\gamma}}{x_{0}^{\frac{3}{4}}}
$$

3. Virial type estimate on $v(t)$. Let $\frac{1}{3}<\theta<\frac{1}{2}, B>1$ to be chosen later and set

$$
I(t)=\frac{1}{2} \int g\left(\frac{x}{\left(B+t^{2}\right)^{\theta}}\right) v^{2}(t, x) d x, \quad z=v \sqrt{g^{\prime}\left(\frac{x}{\left(B+t^{2}\right)^{\theta}}\right)}
$$

where $g(x)=\arctan (x)$.

$$
(\widetilde{\mathcal{L}} z, z)=-2\left(\mathcal{L}\left(z_{x}\right), x z\right)=2 \int\left|D^{\frac{1}{2}} z\right|^{2}+\int z^{2}-\int\left(x Q^{\prime}+Q\right) z^{2} .
$$

For any $0<\sigma_{0}<1$, we claim

$$
\begin{aligned}
& \left|2 I^{\prime}(t)+\frac{1}{\left(B+t^{2}\right)^{\theta}}(\widetilde{\mathcal{L}} z, z)\right| \leq \frac{\sigma_{0}}{\left(B+t^{2}\right)^{\theta}}\|z\|_{L^{2}}^{2}+\frac{C}{\sigma_{0}\left(B+t^{2}\right)^{1-\theta}}\|v\|_{L^{2}}^{2} \\
& +\frac{C}{\left(B+t^{2}\right)^{\frac{7}{4} \theta}}\|z\|_{H^{\frac{1}{2}}}\|v\|_{L^{2}}+\frac{C}{\left(B+t^{2}\right)^{\theta}} \gamma^{\frac{1}{4}}\|z\|_{H^{\frac{1}{2}}}\|v\|_{L^{2}}+\frac{C}{\left(B+t^{2}\right)^{2 \theta}}\|z\|_{L^{2}}^{2} .
\end{aligned}
$$

Proof of (3.13). We compute $I^{\prime}(t)$ :

$$
I^{\prime}(t)=-\frac{\theta t}{\left(B+t^{2}\right)^{\theta+1}} \int x g^{\prime}\left(\frac{x}{\left(B+t^{2}\right)^{\theta}}\right) v^{2}+\int g\left(\frac{x}{\left(B+t^{2}\right)^{\theta}}\right) v v_{t} .
$$

First, note that by Cauchy-Schwarz' inequality, for any $\sigma_{0}>0$,

$$
\begin{aligned}
\mid \frac{\theta t}{\left(B+t^{2}\right)^{\theta+1}} \int x g^{\prime} & \left(\frac{x}{\left(B+t^{2}\right)^{\theta}}\right) v^{2} \mid \leq \frac{\sigma_{0}}{\left(B+t^{2}\right)^{\theta}} \int g^{\prime}\left(\frac{x}{\left(B+t^{2}\right)^{\theta}}\right) v^{2} \\
& +\frac{\theta^{2} t^{2}}{4 \sigma_{0}\left(B+t^{2}\right)^{2-\theta}} \int\left(\frac{x}{\left(B+t^{2}\right)^{\theta}}\right)^{2} g^{\prime}\left(\frac{x}{\left(B+t^{2}\right)^{\theta}}\right) v^{2} .
\end{aligned}
$$


Since $s^{2} g^{\prime}(s) \leq 1$, we obtain

$$
\left|\frac{\theta t}{\left(B+t^{2}\right)^{\theta+1}} \int x g^{\prime}\left(\frac{x}{\left(B+t^{2}\right)^{\theta}}\right) v^{2}\right| \leq \frac{\sigma_{0}}{\left(B+t^{2}\right)^{\theta}} \int z^{2}+\frac{C \theta^{2}}{\sigma_{0}\left(B+t^{2}\right)^{1-\theta}} \int v^{2} .
$$

Second, we use the equation of $v$ to compute the term $\int g\left(\frac{x}{\left(B+t^{2}\right)^{\theta}}\right) v v_{t}$.

$$
\begin{aligned}
& \int g\left(\frac{x}{\left(B+t^{2}\right)^{\theta}}\right) v v_{t} \\
& =\int g\left(\frac{x}{\left(B+t^{2}\right)^{\theta}}\right) v \mathcal{L} v_{x}-\gamma \int g\left(\frac{x}{\left(B+t^{2}\right)^{\theta}}\right) v\left(1-\gamma \partial_{x}^{2}\right)^{-1}\left(2 v_{x x} Q^{\prime}+v_{x} Q^{\prime \prime}\right) \\
& =\mathbf{A}+\mathbf{B} .
\end{aligned}
$$

Estimate on A.

$$
\begin{aligned}
\mathbf{A}= & \int g\left(\frac{x}{\left(B+t^{2}\right)^{\theta}}\right) v\left(-\mathcal{H} v_{x x}+v_{x}\right)-\int g\left(\frac{x}{\left(B+t^{2}\right)^{\theta}}\right) Q v v_{x} \\
= & -\int\left(\frac{1}{\left(B+t^{2}\right)^{\theta}} g^{\prime}\left(\frac{x}{\left(B+t^{2}\right)^{\theta}}\right) v+g\left(\frac{x}{\left(B+t^{2}\right)^{\theta}}\right) v_{x}\right)\left(-\mathcal{H} v_{x}+v\right) \\
& +\frac{1}{2} \int\left(\frac{1}{\left(B+t^{2}\right)^{\theta}} g^{\prime}\left(\frac{x}{\left(B+t^{2}\right)^{\theta}}\right) Q+g\left(\frac{x}{\left(B+t^{2}\right)^{\theta}}\right) Q^{\prime}\right) v^{2} .
\end{aligned}
$$

Next,

$$
\begin{aligned}
\mathbf{A}= & \frac{-1}{\left(B+t^{2}\right)^{\theta}} \int\left|D^{\frac{1}{2}} z\right|^{2}+v\left(D\left(v g^{\prime}\left(\frac{x}{\left(B+t^{2}\right)^{\theta}}\right)-D\left(v \sqrt{g^{\prime}\left(\frac{x}{\left(B+t^{2}\right)^{\theta}}\right)}\right) \sqrt{g^{\prime}\left(\frac{x}{\left(B+t^{2}\right)^{\theta}}\right)}\right)\right. \\
& +\int\left(\mathcal{H} v_{x}\right) v_{x} g\left(\frac{x}{\left(B+t^{2}\right)^{\theta}}\right)-\frac{1}{2} \frac{1}{\left(B+t^{2}\right)^{\theta}} \int z^{2} \\
& +\frac{1}{2} \frac{1}{\left(B+t^{2}\right)^{\theta}} \int\left(x Q^{\prime}+Q\right) z^{2} \\
& +\frac{1}{2} \int\left(g\left(\frac{x}{\left(B+t^{2}\right)^{\theta}}\right)-\frac{x}{\left(B+t^{2}\right)^{\theta}} g^{\prime}\left(\frac{x}{\left(B+t^{2}\right)^{\theta}}\right)\right) Q^{\prime} v^{2} \\
= & -\frac{1}{2} \frac{1}{\left(B+t^{2}\right)^{\theta}}(\widetilde{\mathcal{L}} z, z)+\mathbf{A}_{\mathbf{1}}+\mathbf{A}_{\mathbf{2}}+\mathbf{A}_{\mathbf{3}},
\end{aligned}
$$

where

$$
\begin{aligned}
& \mathbf{A}_{\mathbf{1}}=-\frac{1}{\left(B+t^{2}\right)^{\theta}} \int v\left(D\left(z \sqrt{g^{\prime}\left(\frac{x}{\left(B+t^{2}\right)^{\theta}}\right)}\right)-(D z) \sqrt{g^{\prime}\left(\frac{x}{\left(B+t^{2}\right)^{\theta}}\right)}\right), \\
& \mathbf{A}_{\mathbf{2}}=\int\left(\mathcal{H} v_{x}\right) v_{x} g\left(\frac{x}{\left(B+t^{2}\right)^{\theta}}\right), \\
& \mathbf{A}_{\mathbf{3}}=\frac{1}{2} \int\left(g\left(\frac{x}{\left(B+t^{2}\right)^{\theta}}\right)-\frac{x}{\left(B+t^{2}\right)^{\theta}} g^{\prime}\left(\frac{x}{\left(B+t^{2}\right)^{\theta}}\right)\right) Q^{\prime} v^{2} .
\end{aligned}
$$


Estimate on $\mathbf{A}_{\mathbf{1}}$. By Lemma 14, we have

$$
\left|\mathbf{A}_{1}\right| \leq \frac{C}{\left(B+t^{2}\right)^{\theta}}\|v\|_{L^{2}}\|z\|_{L^{4}}\left\|D \sqrt{g^{\prime}\left(\frac{x}{\left(B+t^{2}\right)^{\theta}}\right)}\right\|_{L^{4}} \leq \frac{C}{\left(B+t^{2}\right)^{\frac{7 \theta}{4}}}\|v\|_{L^{2}}\|z\|_{H^{\frac{1}{2}}} .
$$

Estimate on $\mathbf{A}_{\mathbf{2}}$. Since $\int\left(\mathcal{H} v_{x}\right) v_{x}=0$, Lemma 3, applied to $A=\left(B+t^{2}\right)^{\theta}$ gives

$$
\left|\mathbf{A}_{2}\right| \leq \frac{C}{\left(B+t^{2}\right)^{2 \theta}}\|z\|_{L^{2}}^{2} .
$$

Estimate on $\mathbf{A}_{\mathbf{3}}$. Since for all $y \in \mathbb{R},\left|\arctan y-\frac{y}{1+y^{2}}\right| \leq C y^{2}$, we have, for all $x \in \mathbb{R}$,

$$
\begin{aligned}
\mid\left(g\left(\frac{x}{\left(B+t^{2}\right)^{\theta}}\right)-\frac{x}{\left(B+t^{2}\right)^{\theta}}\right. & \left.g^{\prime}\left(\frac{x}{\left(B+t^{2}\right)^{\theta}}\right)\right) Q^{\prime}(x) \mid \\
& \leq \frac{x^{2}\left|Q^{\prime}(x)\right|}{\left(B+t^{2}\right)^{2 \theta}} \leq \frac{C}{\left(B+t^{2}\right)^{2 \theta}} \frac{1}{1+|x|}
\end{aligned}
$$

Thus,

$$
\left|\mathbf{A}_{3}\right| \leq \frac{C}{\left(B+t^{2}\right)^{2 \theta}}\|v\|_{L^{2}}\|z\|_{L^{2}}
$$

Estimate on B. First, we claim the following.

Claim 1. (i) $x\left(1-\gamma \partial_{x}^{2}\right)^{-1} f=\left(1-\gamma \partial_{x}^{2}\right)^{-1}(x f)-2 \gamma\left(1-\gamma \partial_{x}^{2}\right)^{-2}\left(f^{\prime}\right)$.

(ii) $\left\|\left(1-\gamma \partial_{x}^{2}\right)^{-1} f\right\|_{L^{2}}+\gamma^{\frac{1}{2}}\left\|\left(1-\gamma \partial_{x}^{2}\right)^{-1}\left(f^{\prime}\right)\right\|_{L^{2}}+\gamma\left\|\left(1-\gamma \partial_{x}^{2}\right)^{-1}\left(f^{\prime \prime}\right)\right\|_{L^{2}} \leq C\|f\|_{L^{2}}$, $\left\|\left(1-\gamma \partial_{x}^{2}\right)^{-1}\left(f^{\prime \prime}\right)\right\|_{L^{2}} \leq C \gamma^{-\frac{3}{4}}\|f\|_{\dot{H}^{\frac{1}{2}}}$.

Proof of Claim 1. (i) Let $h=\left(1-\gamma \partial_{x}^{2}\right)^{-1} f$. Then, $x h-\gamma(x h)^{\prime \prime}=x f-2 \gamma h^{\prime}$ and so $x h=\left(1-\gamma \partial_{x}^{2}\right)^{-1}\left(x f-2 \gamma\left(1-\gamma \partial_{x}^{2}\right)^{-1} f^{\prime}\right)$.

(ii) $\int|f|^{2}=\int\left|h-\gamma h^{\prime \prime}\right|^{2}=\int h^{2}+2 \gamma \int\left(h^{\prime}\right)^{2}+\gamma^{2} \int\left(h^{\prime \prime}\right)^{2}$, which proves the first estimate.

Next,

$$
\left\|\left(1-\gamma \partial_{x}^{2}\right)^{-1} f^{\prime \prime}\right\|_{L^{2}} \leq C\left\|\left(\frac{\xi^{2}}{1+\gamma \xi^{2}}\right) \hat{f}\right\|_{L^{2}} \leq C \gamma^{-\frac{3}{4}}\left\||\xi|^{\frac{1}{2}} \hat{f}\right\|_{L^{2}}
$$

since $\forall \xi \in \mathbb{R}, \forall \gamma>0$,

$$
\frac{\xi^{2}}{1+\gamma \xi^{2}} \leq \gamma^{-\frac{3}{4}}|\xi|^{\frac{1}{2}}
$$

The claim is proved. 
Using (i) of Claim 1, we obtain

$$
\mathbf{B}=-\gamma \int \frac{1}{x} g\left(\frac{x}{\left(B+t^{2}\right)^{\theta}}\right) v\left(1-\gamma \partial_{x}^{2}\right)^{-1} H,
$$

where

$$
H=2 x v_{x x} Q^{\prime}+x v_{x} Q^{\prime \prime}-2 \gamma\left(1-\gamma \partial_{x}^{2}\right)^{-1}\left(2 v_{x x} Q^{\prime}+v_{x} Q^{\prime \prime}\right)_{x} .
$$

Since $|g(y)| \leq C|y|$, for all $y$, we have

$$
|\mathbf{B}| \leq \frac{C \gamma}{\left(B+t^{2}\right)^{\theta}}\|v\|_{L^{2}}\left\|\left(1-\gamma \partial_{x}^{2}\right)^{-1} H\right\|_{L^{2}}
$$

Now, we use Claim 1 (ii) to estimate $\left\|\left(1-\gamma \partial_{x}^{2}\right)^{-1} H\right\|_{L^{2}}$. We can rewrite $H$ under the form:

$$
H=\left(2 v x Q^{\prime}\right)^{\prime \prime}+\left(v F_{1}\right)^{\prime}+v F_{2}-2 \gamma\left(1-\gamma \partial_{x}^{2}\right)^{-1}\left(\left(2 v Q^{\prime}\right)^{\prime \prime}+\left(v F_{3}\right)^{\prime}+v F_{4}\right)_{x},
$$

where for $j=1, \ldots, 4,\left|F_{j}(x)\right| \leq C \frac{1}{1+x^{2}}$. Thus,

$$
\begin{aligned}
\left\|\left(1-\gamma \partial^{2}\right)^{-1} H\right\|_{L^{2}} \leq & C \gamma^{-\frac{3}{4}}\left\|v x Q^{\prime}\right\|_{\dot{H}^{\frac{1}{2}}}+C \gamma^{-\frac{1}{2}}\left\|v \frac{1}{1+x^{2}}\right\|_{L^{2}} \\
& +\gamma^{\frac{1}{2}}\left\|\left(1-\gamma \partial_{x}^{2}\right)^{-1}\left(\left(2 v Q^{\prime}\right)^{\prime \prime}+\left(v F_{3}\right)^{\prime}+v F_{4}\right)\right\|_{L^{2}} \\
\leq & C \gamma^{-\frac{3}{4}}\left\|v x Q^{\prime}\right\|_{\dot{H}^{\frac{1}{2}}}+C \gamma^{-\frac{1}{2}}\left\|v \frac{1}{1+x^{2}}\right\|_{L^{2}}
\end{aligned}
$$

Now, we claim

$$
\left\|v \frac{1}{1+x^{2}}\right\|_{L^{2}} \leq C\|z\|_{L^{2}}, \quad\left\|v x Q^{\prime}\right\|_{\dot{H}^{\frac{1}{2}}} \leq C\|z\|_{H^{\frac{1}{2}}} .
$$

The first estimate is clear since $\frac{1}{1+x^{2}} \leq C \sqrt{g^{\prime}}$. Let

$$
f(x)=\frac{x Q^{\prime}(x)}{\sqrt{g^{\prime}\left(\frac{x}{\left(B+t^{2}\right)^{\theta}}\right)}} .
$$

Then, by Lemma 14,

$\left\|D^{\frac{1}{2}}\left(v x Q^{\prime}\right)\right\|_{L^{2}}=\left\|D^{\frac{1}{2}}(z f)\right\|_{L^{2}} \leq\left\|\left(D^{\frac{1}{2}} z\right) f\right\|_{L^{2}}+C\|z\|_{L^{4}}\left\|D^{\frac{1}{2}} f\right\|_{L^{4}} \leq C\|z\|_{H^{\frac{1}{2}}}$, since $\|f\|_{L^{\infty}}+\left\|D^{\frac{1}{2}} f\right\|_{L^{4}} \leq\|f\|_{H^{1}} \leq C$.

Thus, $\left\|\left(1-\gamma \partial^{2}\right)^{-1} H\right\|_{L^{2}} \leq C \gamma^{-\frac{3}{4}}\|z\|_{H^{\frac{1}{2}}}$ and in conclusion for the term $\mathbf{B}$ :

$$
|\mathbf{B}| \leq \frac{C \gamma^{\frac{1}{4}}}{\left(B+t^{2}\right)^{\theta}}\|z\|_{H^{\frac{1}{2}}}\|v\|_{L^{2}} .
$$

Putting together the above estimates, we obtain (3.13).

We now claim the following (see proof in Section 3.2): 
Proposition 3. There exist $\lambda>0, \gamma_{0}>0$ and $B_{0}>1$ such that, for $0<\gamma<\gamma_{0}, B \geq B_{0}$,

$$
\forall t, \quad(\widetilde{\mathcal{L}} z(t), z(t)) \geq \lambda\|z(t)\|_{H^{\frac{1}{2}}}^{2}, \quad \text { where } z \text { is as above. }
$$

Remark 4. The operator $\widetilde{\mathcal{L}}$ does not depend on $\gamma$ and $B$, but the orthogonality conditions on $w$ imply almost orthogonality conditions on $z$ that depend on $\gamma, B$, see proof of Proposition 3.

Choose $\theta=\frac{2}{5}$ and fix $\sigma_{0}=\frac{\lambda}{4}$. Then,

$$
-2 I^{\prime}(t) \geq \frac{\lambda}{2\left(B+t^{2}\right)^{\theta}}\|z(t)\|_{H^{\frac{1}{2}}}^{2}-\frac{C}{\left(B+t^{2}\right)^{\theta}}\left(\frac{1}{\left(B+t^{2}\right)^{\frac{1}{5}}}+\gamma^{\frac{1}{2}}\right)\|v\|_{L^{2}}^{2} .
$$

By the decay property (3.6),

$$
\int v^{2}(t) \leq \int_{|x| \leq \frac{1}{2}\left(B+t^{2}\right)^{\theta}} v^{2}(t)+\frac{C_{\gamma}}{\left(B+t^{2}\right)^{\frac{3}{4} \theta}} \leq C \int z^{2}(t)+\frac{C_{\gamma}}{\left(B+t^{2}\right)^{\frac{3}{10}}}
$$

For $\gamma>0$ small enough and $B$ large enough, and by $\|v\|_{H^{\frac{1}{2}}} \leq C$, we get

$$
-2 I^{\prime}(t) \geq \frac{\lambda}{4\left(B+t^{2}\right)^{\theta}}\|z(t)\|_{H^{\frac{1}{2}}}^{2}-\frac{C_{\gamma}}{\left(B+t^{2}\right)^{\frac{3}{5}}} .
$$

Since $I(t)$ is bounded, we obtain by integration

$$
\int_{-\infty}^{+\infty} \frac{1}{\left(B+t^{2}\right)^{\theta}}\|z(t)\|_{H^{\frac{1}{2}}}^{2} d t<C_{\gamma}
$$

We claim that (3.16) and (3.6) imply

$$
\int_{-\infty}^{+\infty} \frac{1}{\left(B+t^{2}\right)^{\theta}}\|v(t)\|_{H^{\frac{1}{2}}}^{2} d t<C .
$$

Indeed, by (3.6) and the expression of $g^{\prime}$, and considering the two regions $x>\frac{1}{\left(B+t^{2}\right)^{\frac{\theta}{2}}}, x<\frac{1}{\left(B+t^{2}\right)^{\frac{\theta}{2}}}$, we have

$$
\|v-z\|_{H^{1}}^{2}=\left\|v\left(1-\sqrt{g^{\prime}}\right)\right\|_{H^{1}}^{2} \leq \frac{C}{\left(B+t^{2}\right)^{\frac{3}{8} \theta}}=\frac{C}{\left(B+t^{2}\right)^{\frac{3}{20}}} .
$$

Thus, by $\|v\|_{H^{\frac{1}{2}}} \leq\|z\|_{H^{\frac{1}{2}}}+\|v-z\|_{H^{\frac{1}{2}}}$, and (3.16)

$$
\int_{-\infty}^{+\infty} \frac{1}{\left(B+t^{2}\right)^{\theta}}\|v(t)\|_{H^{\frac{1}{2}}}^{2} d t \leq 2 C_{\gamma}+\int_{-\infty}^{+\infty} \frac{1}{\left(B+t^{2}\right)^{\frac{11}{20}}} d t \leq C .
$$


Using another virial argument, we claim

$$
\int_{-\infty}^{+\infty} \frac{1}{\left(B+t^{2}\right)^{\theta}}\|z(t)\|_{H^{\frac{3}{2}}}^{2} d t<C .
$$

Proof of (3.19). We set

$$
J(t)=\frac{1}{2} \int g\left(\frac{x}{\left(B+t^{2}\right)^{\theta}}\right) v_{x}^{2}(t) .
$$

Proceeding as in the proof of (3.13) (the equation for $v_{x}$ is very similar to the one for $v$ ), we obtain

$$
\left|J^{\prime}(t)+\frac{1}{\left(B+t^{2}\right)^{\theta}} \int\left(D^{\frac{3}{2}} z\right)^{2}\right| \leq \frac{C}{\left(B+t^{2}\right)^{\theta}}\|v\|_{H^{1}}\left(\|v\|_{H^{1}}+\|z\|_{H^{\frac{3}{2}}}\right) .
$$

Using $\|v\|_{H^{1}} \leq\|z\|_{H^{1}}+\|v-z\|_{H^{1}},(3.18)$ and the following estimate

$$
\|z\|_{H^{1}} \leq \varepsilon\left\|D^{\frac{3}{2}} z\right\|_{L^{2}}+C_{\varepsilon}\|z\|_{L^{2}}
$$

we obtain, for $\varepsilon>0$ small enough,

$$
-J^{\prime}(t) \geq \frac{1}{2} \frac{1}{\left(B+t^{2}\right)^{\theta}}\left\|D^{\frac{3}{2}} z\right\|_{L^{2}}^{2}+C \frac{1}{\left(B+t^{2}\right)^{\theta}}\|z\|_{L^{2}}^{2} .
$$

Since $J(t)$ is bounded and using (3.16), we obtain (3.19).

Finally, by (3.16), (3.18) and (3.19), we get (3.7). Lemma 4 is proved.

Lemma 5 (Decay estimate on $w(t)$ ). The following hold

$$
\begin{gathered}
\int_{-\infty}^{+\infty} \frac{1}{\left(1+t^{2}\right)^{\frac{2}{5}}}\|w(t)\|_{L^{2}}^{2} d t<C, \\
\sup _{t \in \mathbb{R}} \int|x| w^{2}(t, x) d x \leq C .
\end{gathered}
$$

Proof of Lemma 5. Estimate (3.20) is a consequence of Lemma 4 by comparing $v$ and $w$. Let $\gamma>0$ small. We have by the definition of $v$ : $\left(1-\gamma \partial_{x}^{2}\right) v=\mathcal{L} w$. Let $\widetilde{w}=\left(1-\gamma \partial_{x}^{2}\right)^{-\frac{1}{4}} w$. Then,

$$
\int w\left(1-\gamma \partial_{x}^{2}\right)^{\frac{1}{2}} v=\int \widetilde{w}\left(1-\gamma \partial_{x}^{2}\right)^{-\frac{1}{4}}(\mathcal{L} w) .
$$

On the one hand, we have

$$
\left|\int w\left(1-\gamma \partial_{x}^{2}\right)^{\frac{1}{2}} v\right| \leq C\|w\|_{L^{2}}\|v\|_{H^{1}}
$$


On the other hand, as in the proof of Claim 1

$$
\begin{aligned}
\|\left(1-\gamma \partial_{x}^{2}\right)^{-\frac{1}{4}} & (\mathcal{L} w)-\mathcal{L} \widetilde{w} \|_{L^{2}} \\
& \leq\left\|\left(1-\gamma \partial_{x}^{2}\right)^{-\frac{1}{4}}(Q w)-Q w\right\|_{L^{2}}+\|Q(w-\widetilde{w})\|_{L^{2}} \leq \gamma^{\frac{1}{4}}\|w\|_{L^{2}}
\end{aligned}
$$

Thus,

$$
\left|\int \widetilde{w}\left(1-\gamma \partial_{x}^{2}\right)^{-\frac{1}{4}}(\mathcal{L} w)-(\mathcal{L} \widetilde{w}, \widetilde{w})\right| \leq C \gamma^{\frac{1}{4}}\|w\|_{L^{2}}^{2}
$$

and since $(\mathcal{L} \widetilde{w}, \widetilde{w}) \geq \frac{1}{2} \lambda\|\widetilde{w}\|_{H^{\frac{1}{2}}}$ for $\gamma>0$ small enough (this is a consequence of Lemma 15 and the orthogonality conditions on $w$-see Section 3.2, in particular the proof of Proposition 3), we obtain

$$
\int \widetilde{w}\left(1-\gamma \partial_{x}^{2}\right)^{-\frac{1}{4}}(\mathcal{L} w) \geq \frac{\lambda}{2}\|\widetilde{w}\|_{H^{\frac{1}{2}}}^{2}-C \gamma^{\frac{1}{4}}\|w\|_{L^{2}}^{2} \geq \lambda_{1}\|w\|_{L^{2}}^{2}
$$

In conclusion, we have obtained

$$
\|w\|_{L^{2}} \leq C\|v\|_{H^{1}}
$$

and Lemma 4 then implies (3.20).

Now, we prove (3.21). Indeed, the integrability property (3.20) allows us to obtain the decay on $w(t, x)$ by monotonicity properties.

By the proof of Proposition 2, we have, for any $\lambda \in(0,1)$, for any $t_{0}$, $t \in\left(-\infty, t_{0}\right], x_{0}>1$,

$$
\begin{aligned}
& \int w^{2}\left(t_{0}, x\right)\left(\varphi\left(x-x_{0}\right)-\varphi\left(-x_{0}\right)\right) d x \\
& \leq \int w^{2}(t, x)\left(\varphi\left(x-x_{0}-\lambda\left(t_{0}-t\right)\right)-\varphi\left(-x_{0}-\lambda\left(t_{0}-t\right)\right)\right) d x \\
& \quad+C \int_{t}^{t_{0}} \frac{\left\|w\left(t^{\prime}\right)\right\|_{L^{2}}^{2} d t^{\prime}}{\left(x_{0}+\lambda\left(t_{0}-t^{\prime}\right)\right)^{2}}
\end{aligned}
$$

The last term in (3.22) is treated as follows $\left(x_{0}>1\right)$

$$
\int_{t}^{t_{0}} \frac{\left\|w\left(t^{\prime}\right)\right\|_{L^{2}}^{2} d t^{\prime}}{\left(x_{0}+\lambda\left(t_{0}-t^{\prime}\right)\right)^{2}} \leq C x_{0}^{-\frac{6}{5}} \int_{-\infty}^{+\infty} \frac{\left\|w\left(t^{\prime}\right)\right\|_{L^{2}}^{2} d t^{\prime}}{\left(1+\left(t_{0}-t^{\prime}\right)\right)^{\frac{4}{5}}}
$$

Thus, by (3.20) (applied to $w\left(t+t_{0}\right)$ ) and (3.3), letting $t \rightarrow-\infty$ in (3.22), we obtain

$$
\int w^{2}\left(t_{0}\right)\left(\varphi\left(x-x_{0}\right)-\varphi\left(-x_{0}\right)\right) d x \leq C x_{0}^{-\frac{6}{5}} .
$$


By the change of variable $x \rightarrow-x, t \rightarrow-t$, which leaves the equation invariant, we get:

$$
\int w^{2}\left(t_{0}\right)\left(\varphi\left(x_{0}\right)-\varphi\left(x+x_{0}\right)\right) d x \leq C x_{0}^{-\frac{6}{5}}
$$

and thus, summing up the two estimates,

$$
\int w^{2}\left(t_{0}\right)\left(\varphi\left(x-x_{0}\right)-\varphi\left(x+x_{0}\right)+\varphi\left(x_{0}\right)-\varphi\left(-x_{0}\right)\right) d x \leq C x_{0}^{-\frac{6}{5}} .
$$

We verify easily that for all $|x|>x_{0} \geq 1$,

$$
\begin{aligned}
& \varphi\left(x-x_{0}\right)-\varphi\left(x+x_{0}\right)+\varphi\left(x_{0}\right)-\varphi\left(-x_{0}\right) \\
& \quad \geq \varphi(0)-\varphi\left(2 x_{0}\right)+\varphi\left(x_{0}\right)-\varphi\left(-x_{0}\right) \geq \frac{\pi}{2}-\arctan (2)>0 .
\end{aligned}
$$

Thus, for all $x_{0}>1$,

$$
\int_{|x| \geq x_{0}} w^{2}\left(t_{0}\right) \leq C x_{0}^{-\frac{6}{5}}
$$

By integrating in $x_{0}$, we obtain the following estimate

$$
\forall t \in \mathbb{R}, \quad \int|x| w^{2}(t) \leq C .
$$

Thus Lemma 5 is proved.

Now, we claim that estimate (3.21) implies a gain of regularity on $w(t)$.

Lemma 6 (Gain of regularity on $w(t)$ ). Let $w \in C\left(\mathbb{R}, L^{2}(\mathbb{R})\right) \cap L^{\infty}\left(\mathbb{R}, L^{2}(\mathbb{R})\right.$ ) be a solution of (3.1) satisfying (3.21). Then, $w(t) \in C\left(\mathbb{R}, H^{\frac{1}{2}}(\mathbb{R})\right)$ and the following identity holds

$$
\begin{aligned}
\int x w^{2}\left(t_{2}\right)-\int x w^{2}\left(t_{1}\right)= & -\int_{t_{1}}^{t_{2}} \int\left(2\left|D^{\frac{1}{2}} w\right|^{2}+w^{2}+w^{2}\left(x Q^{\prime}-Q\right)\right) \\
& +2 \int_{t_{1}}^{t_{2}} \beta(t) \int x Q^{\prime} w .
\end{aligned}
$$

End of the proof of Theorem 3 assuming Lemma 6 . Note first that multiplying the equation of $w(t)$ by $Q^{\prime}$ and using $\int w Q^{\prime}=0$, we find $\left(\int\left(Q^{\prime}\right)^{2}\right) \beta(t)=\int w \mathcal{L}\left(Q^{\prime \prime}\right)$, so that

$$
|\beta(t)| \leq C\|w\|_{L^{2}}
$$

Multiplying the equation of $w(t)$ by $\mathcal{L} w$ and using $\mathcal{L} Q^{\prime}=0$, we also have

$$
\forall t \in \mathbb{R}, \quad(\mathcal{L} w(t), w(t))=(\mathcal{L} w(0), w(0)) .
$$


By (3.26), the estimates on $\int|x| w^{2}(t)$ and on $\beta(t)$, and Lemma 5 , we have

$$
\int_{-\infty}^{+\infty} \frac{1}{\left(1+t^{2}\right)^{\frac{2}{5}}}\|w(t)\|_{H^{\frac{1}{2}}}^{2} d t<C
$$

This implies that for a sequence $t_{n} \rightarrow+\infty$, we have $\left\|w\left(t_{n}\right)\right\|_{H^{\frac{1}{2}}} \rightarrow 0$ as $n \rightarrow+\infty$.

Since $(\mathcal{L} w(t), w(t))=\lim _{t_{n} \rightarrow \infty}\left(\mathcal{L} w\left(t_{n}\right), w\left(t_{n}\right)\right)$, we obtain $(\mathcal{L} w(t), w(t))=0$ and so by the orthogonality conditions on $w(t)$ and Lemma 15 , we finally obtain $\forall t, w(t)=0$.

Proof of Lemma 6. Formally, identity (3.26) follows from multiplying equation (3.1) by $x w$, integration by parts and properties of the Hilbert transform. To justify (3.26), we use a regularization of $w(t)$.

We set $w_{n}=\left(1-\frac{1}{n} \partial_{x}^{2}\right)^{-1} w$, so that for all $t, w_{n}(t) \rightarrow w(t)$ in $L^{2}(\mathbb{R})$ as $n \rightarrow+\infty$. Then, $w_{n}$ satisfies the following equation

$$
w_{n t}=\left(\mathcal{L} w_{n}\right)_{x}-\frac{1}{n}\left(1-\frac{1}{n} \partial_{x}^{2}\right)^{-1}\left(2 Q^{\prime} w_{n x}+w_{n} Q^{\prime \prime}\right)_{x}+\beta\left(1-\frac{1}{n} \partial_{x}^{2}\right)^{-1} Q^{\prime}
$$

Let $h: \mathbb{R} \rightarrow \mathbb{R}$ be a smooth nondecreasing function such that $h(x)=x$ if $x>1$ and $h(x)=0$ if $x<0$. Then,

$$
\begin{aligned}
\int h(x) w^{2} & =\int h(x)\left(w_{n}-\frac{1}{n} w_{n x x}\right)^{2} \\
& =\int h(x) w_{n}^{2}-\frac{2}{n} \int w_{n x x} w_{n} h(x)+\frac{1}{n^{2}} \int w_{n x x}^{2} h(x) \\
& =\int h(x) w_{n}^{2}+\frac{2}{n} \int w_{n x}^{2} h(x)-\frac{1}{n} \int w_{n}^{2} h^{\prime \prime}(x)+\frac{1}{n^{2}} \int w_{n x x}^{2} h(x)
\end{aligned}
$$

implies that

$$
\int_{x>0} x w_{n}^{2} \leq C \quad \text { and } \quad \int_{x>0} x\left(w-w_{n}\right)^{2} \rightarrow 0 \quad \text { as } n \rightarrow+\infty .
$$

The same holds true in the region $x<0$.

For the functions $w_{n}$, we have the following identity, for any $t_{1}<t_{2}$ :

$$
\begin{aligned}
\int x w_{n}^{2}\left(t_{2}\right)-\int x w_{n}^{2}\left(t_{1}\right)= & -\int_{t_{1}}^{t_{2}} \int\left(2\left|D^{\frac{1}{2}} w_{n}\right|^{2}+w_{n}^{2}+w_{n}^{2}\left(x Q^{\prime}-Q\right)\right) d x d t \\
& +\int_{t_{1}}^{t_{2}} \int\left(-\frac{2}{n} x\left(1-\frac{1}{n} \partial_{x}^{2}\right)^{-1}\left(2 Q^{\prime} w_{n x}+w_{n} Q^{\prime \prime}\right)_{x} w_{n}\right) \\
& +2 \int_{t_{1}}^{t_{2}} \beta \int x\left(\left(1-\frac{1}{n} \partial_{x}^{2}\right)^{-1} Q^{\prime}\right) w_{n} d x d t
\end{aligned}
$$


Indeed, multiplying the equation of $w_{n}$ by $A g\left(\frac{x}{A}\right) w_{n}$ where $g(x)=\arctan (x)$, we find

$$
\begin{aligned}
\int A g\left(\frac{x}{A}\right) w_{n}^{2}\left(t_{2}\right)-\int A g\left(\frac{x}{A}\right) w_{n}^{2}\left(t_{1}\right) \\
=-\int_{t_{1}}^{t_{2}} \int\left(2\left|D^{\frac{1}{2}} w_{n}\right|^{2} g^{\prime}\left(\frac{x}{A}\right)+2 D^{\frac{1}{2}} w_{n}\left(D^{\frac{1}{2}}\left(w_{n} g^{\prime}\left(\frac{x}{A}\right)\right)-D^{\frac{1}{2}}\left(w_{n}\right) g^{\prime}\left(\frac{x}{A}\right)\right)\right. \\
\left.\quad+2 D w_{n} w_{n x} A g\left(\frac{x}{A}\right)+w_{n}^{2} g^{\prime}\left(\frac{x}{A}\right)+w_{n}^{2}\left(A g\left(\frac{x}{A}\right) Q^{\prime}-g^{\prime}\left(\frac{x}{A}\right) Q\right)\right) d x d t \\
\quad-2 \int_{t_{1}}^{t_{2}} \beta(t) \int x\left(\left(1-\frac{1}{n} \partial_{x}^{2}\right)^{-1} Q\right) A g\left(\frac{x}{A}\right) w_{n} \\
\quad-\frac{2}{n} \int_{t_{1}}^{t_{2}} \int\left(\left(1-\frac{1}{n} \partial_{x}^{2}\right)^{-1} Q\right)\left(2 Q^{\prime} w_{n x}+w_{n} Q^{\prime \prime}\right)_{x} A g\left(\frac{x}{A}\right) w_{n} .
\end{aligned}
$$

Then, (3.30) is proved using Lemmas 3 and 14 (see the proof of Lemma 4 for similar arguments) and then passing to the limit as $A \rightarrow+\infty$ applying the Lebesgue convergence theorem.

From (3.30), we claim that for any $t_{1}, t_{2}$,

$$
\limsup _{n \rightarrow+\infty} \int_{t_{1}}^{t_{2}}\left\|w_{n}(t)\right\|_{H^{\frac{1}{2}}}^{2} d t<+\infty .
$$

Proof of (3.31). By Claim 1 (i), we have

$$
\begin{aligned}
\frac{1}{n} \int x\left(1-\frac{1}{n} \partial_{x}^{2}\right)^{-1} & \left(2 Q^{\prime} w_{n x}+w_{n} Q^{\prime \prime}\right)_{x} w_{n} \\
= & \frac{1}{n} \int w_{n}\left(1-\frac{1}{n} \partial_{x}^{2}\right)^{-1}\left(2 x Q^{\prime} w_{n x x}+3 x Q^{\prime \prime} w_{n x}+x Q^{(3)} w_{n}\right) \\
& -\frac{2}{n^{2}} \int w_{n}\left(1-\frac{1}{n} \partial_{x}^{2}\right)^{-2}\left(2 Q^{\prime} w_{n x}+w_{n} Q^{\prime \prime}\right)_{x x}=\mathbf{I}+\mathbf{I I} .
\end{aligned}
$$

As in the proof of Lemma 4 (control of $\mathbf{B}$ ), we have

$$
|\mathbf{I}| \leq \frac{C}{n^{\frac{1}{4}}}\left\|w_{n}\right\|_{H^{\frac{1}{2}}}\left\|w_{n}\right\|_{L^{2}}, \quad|\mathbf{I I}| \leq \frac{C}{n^{\frac{1}{2}}}\left\|w_{n}\right\|_{L^{2}}^{2} .
$$

From (3.30), (3.27), the $L^{2}$ bounds on $w(t)$ and $w_{n}(t)$ and (3.32) we obtain

$$
\int_{t_{1}}^{t_{2}}\left\|w_{n}(t)\right\|_{H^{\frac{1}{2}}}^{2} d t \leq C\left|t_{2}-t_{1}\right|+\sup _{t} \int|x| w_{n}^{2}(t)+\frac{C}{n^{\frac{1}{4}}} \int_{t_{1}}^{t_{2}}\left\|w_{n}\right\|_{H^{\frac{1}{2}}}^{2} d t .
$$

For $n$ large enough, we get $\int_{t_{1}}^{t_{2}}\left\|w_{n}(t)\right\|_{H^{\frac{1}{2}}}^{2} d t \leq C$. Thus (3.31) is proved.

By the well-posedness of the equation of $w(t)$ in $H^{\frac{1}{2}}$, we obtain $\forall t, w(t) \in$ $H^{\frac{1}{2}}$ and $w_{n} \rightarrow w$ in $H^{\frac{1}{2}}$. Finally, from (3.29) and (3.32), we obtain (3.26) by passing to the limit as $n \rightarrow \infty$ in (3.30). 


\subsection{Positivity of a quadratic form related to the dual problem}

In this section, we prove Proposition 3. The main ingredient is the following result.

Proposition 4. There exists $\lambda_{0}>0$ such that for all $z \in H^{\frac{1}{2}}$,

$$
\int z(x Q)^{\prime}=0 \Rightarrow
$$

$$
(\widetilde{\mathcal{L}} z, z)=2 \int\left|D^{\frac{1}{2}} z\right|^{2}+\int z^{2}-\int\left(x Q^{\prime}+Q\right) z^{2} \geq \lambda_{0}\|z\|_{H^{\frac{1}{2}}}^{2} .
$$

Proof of Proposition 4. First, we introduce some notation. Recall that

$$
\mathcal{L} f=-\mathcal{H} f^{\prime}+f-Q f .
$$

We define $S=(x Q)^{\prime}$. Note that $S=\frac{d}{d c} Q_{c \mid c=1}$ and thus by differentiating the equation of $Q_{c}$ with respect to $c$, and taking $c=1$, we find $\mathcal{L} S=-Q$. Observe also that $\mathcal{L} Q=-\mathcal{H} Q^{\prime}+Q-Q^{2}=-\frac{1}{2} Q^{2}$, by the equation of $Q$. Now, we set $T=S-Q$. Then, $\mathcal{L} T=-Q+\frac{1}{2} Q^{2}=(x Q)^{\prime}=S$, by using the explicit expression $Q(x)=\frac{4}{1+x^{2}}$. We compute $\int T S=\int S^{2}-\int Q S$. Since $S=\mathcal{H} Q^{\prime}=\frac{1}{2} Q^{2}-Q$ (explicit computation), we have $\int S^{2}=\int\left(Q^{\prime}\right)^{2}$ and $\left(Q^{\prime}\right)^{2}=\frac{64 x^{2}}{\left(1+x^{2}\right)^{4}}=Q^{3}-\frac{1}{4} Q^{4}$, thus

$\int\left(Q^{\prime}\right)^{2}=\int Q^{3}-\frac{1}{4} \int Q^{4}=\int S^{2}=\int\left(\frac{1}{2} Q^{2}-Q\right)^{2}=\frac{1}{4} \int Q^{4}-\int Q^{3}+\int Q^{2}$, we find $\int S^{2}=\frac{1}{2} \int Q^{2}$. Moreover, $\int S Q=-\int x Q Q^{\prime}=\frac{1}{2} \int Q^{2}$, and so $\int T S=0$. Finally, $\int T Q=-\int T \mathcal{L} S=-\int S^{2}$.

In conclusion, we have proved $\left((.,\right.$.$) denotes the L^{2}$ scalar product):

$S=\frac{1}{2} Q^{2}-Q=(x Q)^{\prime}, T=S-Q, \mathcal{L} Q=-\frac{1}{2} Q^{2}, \mathcal{L} S=-Q, \mathcal{L} T=S$,

$$
(S, Q)=\frac{1}{2} \int Q^{2},(S, T)=0,(T, Q)=-\int S^{2} .
$$

Now, we claim the following.

Lemma 7. There exists $\lambda>0$ such that, for all $\varepsilon>0$, if $\int w S_{\varepsilon}=0$, where $S_{\varepsilon}=S+\varepsilon Q$, then $(\mathcal{L} w, w) \geq 0$ and $(\widetilde{\mathcal{L}} w, w) \geq \lambda\|w\|_{H^{\frac{1}{2}}}^{2}$.

Proof of Lemma 7. Let $T_{\varepsilon}=T-\varepsilon S$ and $S_{\varepsilon}=S+\varepsilon Q$, then by (3.35): $\mathcal{L} T_{\varepsilon}=S_{\varepsilon}$ and

$$
\left(\mathcal{L} T_{\varepsilon}, T_{\varepsilon}\right)=\left(S_{\varepsilon}, T_{\varepsilon}\right)=(S, T)+\varepsilon(-(S, S)+(T, Q))-\varepsilon^{2}(S, Q) \leq-2 \varepsilon(S, S)<0 .
$$

Moreover, it is clear that if $f_{0}, \lambda_{0}$ denote respectively the first eigenfunction and first eigenvalue of $\mathcal{L}$ (see Lemma 15) we have $\left(S, f_{0}\right)=\left(\mathcal{L} T, f_{0}\right)=$ $\left(T, \mathcal{L} f_{0}\right)=\lambda_{0}\left(x Q^{\prime}, f_{0}\right) \neq 0$, since $f_{0}>0$. Thus, by Lemma E.1 in [29], we obtain the first part of Lemma 7. 
Now, we note that since $x Q^{\prime}>0$,

$$
\begin{aligned}
(\widetilde{\mathcal{L}} w, w) & =2 \int\left|D^{\frac{1}{2}} w\right|^{2}+\int w^{2}-\int\left(x Q^{\prime}+Q\right) w^{2} \\
& \geq 2 \int\left|D^{\frac{1}{2}} w\right|^{2}+\int w^{2}-\int Q w^{2}=\int\left|D^{\frac{1}{2}} w\right|^{2}+(\mathcal{L} w, w) .
\end{aligned}
$$

Using the inequality $\|w\|_{L^{4}}^{2} \leq C\|w\|_{L^{2}}\left\|D^{\frac{1}{2}} w\right\|_{L^{2}}$ (see (A.1)) and CauchySchwarz' inequality, we have, for some constant $C_{0}>0$,

$$
\int Q w^{2} \leq C\left\|w^{2}\right\|_{L^{2}} \leq C_{0} \int\left|D^{\frac{1}{2}} w\right|^{2}+\frac{1}{2} \int w^{2} .
$$

Thus, for $\delta_{0}>0$ such that $2-C_{0} \delta_{0}>1-\frac{\delta_{0}}{2}$, we have

$$
\begin{aligned}
(\widetilde{\mathcal{L}} w, w) & \geq\left(2-C_{0} \delta_{0}\right) \int\left|D^{\frac{1}{2}} w\right|^{2}+\left(1-\frac{1}{2} \delta_{0}\right) \int w^{2}-\left(1-\delta_{0}\right) \int Q w^{2} \\
& \geq\left(1-\delta_{0}\right)(\mathcal{L} w, w)+\frac{\delta_{0}}{2}\|w\|_{H^{\frac{1}{2}}}^{2} \geq \frac{\delta_{0}}{2}\|w\|_{H^{\frac{1}{2}}}^{2}
\end{aligned}
$$

provided $\int w S_{\varepsilon}=0$.

Now, we finish the proof of Proposition 4. Let $z \in H^{\frac{1}{2}}$ be such that $\int z S=\int z(x Q)^{\prime}=0$. Let $w=z+a Q$, where $\int w S_{\varepsilon}=0,0<\varepsilon<\varepsilon_{0}$, where $\varepsilon_{0}$ is to be chosen small enough. In particular, we have

$$
\begin{aligned}
\int w S_{\varepsilon} & =\int z S_{\varepsilon}+a \int Q S_{\varepsilon}=\varepsilon \int z Q+a \int S Q+a \varepsilon \int Q^{2} \\
& =\varepsilon \int z Q+a\left(\frac{1}{2}+\varepsilon\right) \int Q^{2}=0,
\end{aligned}
$$

and so $|a| \leq \frac{2}{\|Q\|_{L^{2}}} \varepsilon\|z\|_{L^{2}}$, and $\|w\|_{L^{2}} \leq 2\|z\|_{L^{2}}$ for $\varepsilon_{0}$ small enough. Similarly, we have $\|z\|_{L^{2}} \leq 2\|w\|_{L^{2}}$, by possibly choosing a smaller $\varepsilon_{0}$. By Lemma 7 , we obtain

$$
\frac{\lambda}{2}\|z\|_{H^{\frac{1}{2}}}^{2} \leq \lambda\|w\|_{H^{\frac{1}{2}}}^{2} \leq(\widetilde{\mathcal{L}} w, w)=(\widetilde{\mathcal{L}} z, z)+a^{2}(\widetilde{\mathcal{L}} Q, Q)+2 a(\widetilde{\mathcal{L}} Q, z) .
$$

For $\varepsilon_{0}$ small, we get $(\widetilde{\mathcal{L}} z, z) \geq \frac{\lambda}{4}\|z\|_{H^{\frac{1}{2}}}^{2}$.

Now, we are in a position to prove Proposition 3.

Proof of Proposition 3. In Proposition 3, we want to prove that for $B$ large and $\gamma$ small, and for some $\lambda_{1}>0$, for all $t$,

$$
(\widetilde{\mathcal{L}} z(t), z(t)) \geq \lambda_{1}\|z(t)\|_{H^{\frac{1}{2}}}^{2}, \quad \text { for } \quad z(t)=v(t) \sqrt{g^{\prime}\left(\frac{x}{\left(B+t^{2}\right)^{\alpha}}\right)},
$$

where $v=\left(1-\gamma \partial_{x}^{2}\right)^{-1}(\mathcal{L} w)$. Formally, if $B=+\infty$ and $\gamma=0$, we have $z(t)=v(t)=\mathcal{L} w$ and $0=\int w Q=-\int w \mathcal{L} S=-\int z S$, and the result follows from Proposition 4. Now, we justify that the result persists for large values of $B$ and small values of $\gamma$. 
Let $S_{B, \gamma}(t)=\left(g^{\prime}\left(\frac{x}{\left(B+t^{2}\right)^{\alpha}}\right)\right)^{-\frac{1}{2}}\left(S-\gamma S^{\prime \prime}\right)$. Then,

$$
\mathcal{L}\left(\left(1-\gamma \partial_{x}^{2}\right)^{-1}\left(\sqrt{g^{\prime}\left(\frac{x}{\left(B+t^{2}\right)^{\alpha}}\right)} S_{B, \gamma}(t)\right)=-Q\right.
$$

and so $\int S_{B, \gamma}(t) z=-\int w Q=0$. Now, we control $S_{B, \gamma}(t)-S$ :

$$
\begin{aligned}
S_{B, \gamma}(t)-S & =\sqrt{1+\frac{x^{2}}{\left(B+t^{2}\right)^{\alpha}}}\left(S-\gamma S^{\prime \prime}\right)-S \\
& =\left(\sqrt{1+\frac{x^{2}}{\left(B+t^{2}\right)^{\alpha}}}-1\right) S-\gamma \sqrt{1+\frac{x^{2}}{\left(B+t^{2}\right)^{\alpha}}} S^{\prime \prime} .
\end{aligned}
$$

Thus, by elementary estimates and the expression of $S$, we obtain:

$$
\left|S_{B, \gamma}(t, x)-S(x)\right| \leq\left(B^{-\frac{\alpha}{2}}+\gamma\right) \frac{1}{1+|x|} .
$$

It follows that

$$
\left|\int S z(t)\right|=\left|\int\left(S-S_{B, \gamma}(t)\right) z(t)\right| \leq\left(B^{-\frac{\alpha}{2}}+\gamma\right)\|z\|_{L^{2}}
$$

Setting $z=z_{1}+a Q$, where $\int z_{1} S=0$ and $|a| \leq\left(B^{-\frac{\alpha}{2}}+\gamma\right)\|z\|_{L^{2}}$, we conclude the proof of Proposition 3 as at the end of the proof of Proposition 4 , for $B$ large enough and $\gamma$ small enough.

\section{Proof of asymptotic stability - Theorem 1}

In this section, we first prove that Theorem 2 implies Theorem 1 . Then, we prove that Theorem 3 (proved in Section 3) implies Theorem 2.

\subsection{Proof of Theorem 1 assuming Theorem 2}

We follow the strategy of [16], [17], the main idea being to use monotonicity type arguments (such as Proposition 1) to prove that a limiting solution of (1.1) has uniform decay in space. See also [18] for similar use of monotonicity arguments.

We consider a solution $u(t)$ of $(1.1)$ in $H^{\frac{1}{2}}$ which satisfies

$$
\left\|u_{0}-Q\right\|_{H^{\frac{1}{2}}}=\alpha<\alpha_{0}, \quad \text { for } \alpha_{0}>0 \text { small enough. }
$$

By the stability property, for all $t \in \mathbb{R}$,

$$
\inf _{y}\|u(t)-Q(.-y)\|_{H^{\frac{1}{2}}} \leq C \alpha .
$$


1. Decomposition of $u(t)$ around the asymptotic soliton. First, we determine the parameter $c^{+}>0$. It is given by the amount of $L^{2}$ norm that remains on the region $x>\frac{t}{10}$ asymptotically as $t \rightarrow+\infty$. Let $\varphi$ be as in (2.6), with $A>1$ so that Proposition 1 holds. Let

$$
c^{+}=\frac{1}{\pi \int Q^{2}} \limsup _{t \rightarrow+\infty} \int u^{2}(t, x) \varphi\left(x-\frac{t}{10}\right) d x .
$$

From the stability property, $\left|c^{+}-1\right| \leq C \alpha_{0}\left(\lim _{+\infty} \varphi=\pi\right)$. Using Lemma 1 to decompose $u(t)$ around $Q_{c^{+}}$, we consider the following decomposition of $u(t)$

$$
\begin{aligned}
& u(t, x)=Q_{c^{+}}(x-\rho(t))+\eta(t, x-\rho(t)), \\
& \int Q_{c^{+}}^{\prime} \eta(t, x) d x=0, \quad \sup _{t}\|\eta(t)\|_{H^{\frac{1}{2}}} \leq K \alpha_{0} .
\end{aligned}
$$

In what follows, we consider $\alpha_{0}>0$ small enough, so that the following holds (by (2.2)):

$$
\forall t, \quad \frac{99}{100} \leq \rho^{\prime}(t) \leq \frac{101}{100}, \quad \frac{99}{100} \leq c^{+} \leq \frac{101}{100} .
$$

2. Monotonicity arguments. We claim the following estimates:

Lemma 8. (Asymptotics on $u(t)$ ).

$$
\forall y_{0}>1, \quad \limsup _{t \rightarrow+\infty} \int u^{2}(t, x) \varphi\left(x-y_{0}-\rho(t)\right) d x \leq \frac{C}{y_{0}}
$$

$\forall y_{0}>1, \quad \limsup _{t \rightarrow+\infty} \int u^{2}(t, x)\left(\varphi\left(x-\rho(t)+\frac{t}{10}\right)-\varphi\left(x-\rho(t)+y_{0}\right)\right) d x \leq \frac{C}{y_{0}}$,

$$
\begin{gathered}
\lim _{t \rightarrow+\infty} \int u^{2}(t, x)\left(\varphi\left(x-\rho(t)+\frac{19}{20} t\right)-\varphi\left(x-\rho(t)+\frac{t}{10}\right)\right) d x=0, \\
\lim _{t \rightarrow+\infty} \int u^{2}(t, x) \varphi\left(x-\rho(t)+\frac{t}{10}\right) d x=c^{+} \pi \int Q^{2} .
\end{gathered}
$$

Proof of Lemma 8. Monotonicity property on the right of the soliton. By (2.7), with $\lambda=\frac{1}{2}$, we have, for all $y_{0}>1$,

$$
\int u^{2}(t, x) \varphi\left(x-y_{0}-\rho(t)\right) d x \leq \int u^{2}(0, x) \varphi\left(x-y_{0}-\rho(0)-\frac{1}{2} t\right) d x+\frac{C}{y_{0}} .
$$

Since $\lim _{t \rightarrow+\infty} \int u^{2}(0, x) \varphi\left(x-y_{0}-\rho(0)-\frac{1}{2} t\right) d x=0$, we obtain (4.4). 
Monotonicity property on the left of the soliton. By (2.8), with $\lambda=\frac{19}{20}$ and $x_{0}=\frac{19}{20} t^{\prime}$, we have for all $0 \leq t^{\prime} \leq t$,

$$
\int u^{2}(t, x) \varphi\left(x-\rho(t)+\frac{19}{20} t\right) d x \leq \int u^{2}\left(t^{\prime}, x\right) \varphi\left(x-\rho\left(t^{\prime}\right)+\frac{19}{20} t^{\prime}\right) d x+\frac{C}{t^{\prime}} .
$$

It follows that $\int u^{2}(t, x) \varphi\left(x-\rho(t)+\frac{19}{20} t\right) d x$ has a limit as $t \rightarrow+\infty$. Set

$$
\ell=\lim _{t \rightarrow+\infty} \int u^{2}(t, x) \varphi\left(x-\rho(t)+\frac{19}{20} t\right) d x, \quad \ell \geq c^{+} \pi \int Q^{2} .
$$

Applying (2.8) with $\lambda=\frac{100}{99}\left(\frac{19}{20}-\frac{1}{1000}\right)<1$ and $x_{0}=\frac{t}{1000}$, we find

$$
\int u^{2}(t, x) \varphi\left(x-\rho(t)+\frac{19}{20} t\right) d x \leq \int u^{2}\left(\frac{t}{100}, x\right) \varphi\left(x-\rho\left(\frac{t}{100}\right)+\frac{t}{1000}\right) d x+\frac{C}{t} .
$$

Since

$$
\limsup _{t \rightarrow+\infty} \int u^{2}\left(\frac{t}{100}, x\right) \varphi\left(x-\rho\left(\frac{t}{100}\right)+\frac{1}{10} \frac{t}{100}\right) d x \leq c^{+} \pi \int Q^{2},
$$

we obtain $c^{+} \pi \int Q^{2}=\ell$ and (4.6).

Fix $y_{0}>1$, pick $\lambda=\frac{1}{2}$. Consider $t_{2}>t$ and define $t_{1}=\frac{4}{5} t_{2}+2 y_{0}$, so that for $t$ large, $t_{1}<t_{2}$. But then, by (2.8),

$$
\begin{aligned}
\int u^{2}\left(t_{2}, x\right) \varphi\left(x-\rho\left(t_{2}\right)+\frac{t_{2}}{10}\right) d x & =\int u^{2}\left(t_{2}, x\right) \varphi\left(x-\rho\left(t_{2}\right)+\lambda\left(t_{2}-t_{2}\right)+y_{0}\right) d x \\
& \leq \int u^{2}\left(t_{1}, x\right) \varphi\left(x-\rho\left(t_{1}\right)+y_{0}\right) d x+\frac{C}{y_{0}} .
\end{aligned}
$$

In light of (4.6) and the existence of $\ell$, (4.5) follows. Thus Lemma 8 is proved.

3. Construction of a compact limit object. Let $t_{n} \rightarrow+\infty$. By the uniform bound on $u(t)$ in $H^{\frac{1}{2}}$, there exist $\widetilde{u}_{0} \in H^{\frac{1}{2}}$ and a subsequence, still denoted by $\left(t_{n}\right)$, such that

$$
u\left(t_{n}, .+\rho\left(t_{n}\right)\right) \rightarrow \widetilde{u}_{0} \quad \text { in } H^{\frac{1}{2}} \text { weak as } n \rightarrow+\infty .
$$

Consider $\widetilde{u}(t)$ the global $H^{\frac{1}{2}}$ solution of (1.1) such that $\widetilde{u}(0)=\widetilde{u}_{0}$. By (4.2), $\left\|\widetilde{u}_{0}-Q_{c^{+}}\right\| \leq C \alpha_{0}$ and thus by the stability property, $\sup _{t} \inf _{y} \| \widetilde{u}(t)-$ $Q(.-y) \|_{H^{\frac{1}{2}}} \leq C \alpha_{0}$. Let $\widetilde{\rho}(t), \widetilde{\eta}(t)$ correspond to the decomposition of $\widetilde{u}(t)$ around $Q_{c^{+}}$given by Lemma 1 .

By Theorem 5 below and Remark 2 , for all $t \in \mathbb{R}$, we have

$$
\begin{aligned}
& u\left(t_{n}+t, .+\rho\left(t_{n}\right)\right) \rightarrow \widetilde{u}(t) \quad \text { in } H^{\frac{1}{2}} \text { weak, } \\
& \rho\left(t_{n}+t\right)-\rho\left(t_{n}\right) \rightarrow \widetilde{\rho}(t) \quad \text { as } n \rightarrow+\infty .
\end{aligned}
$$


From weak convergence and Lemma 8, we claim the following decay estimate on $\widetilde{u}(t)$ :

$$
\forall y_{0}>1, \forall t \in \mathbb{R}, \quad \int_{|x|>y_{0}} \widetilde{u}^{2}(t, x+\widetilde{\rho}(t)) d x \leq \frac{C}{y_{0}}
$$

Indeed, first, from (4.4), for any fixed $y_{0}>1, t \in \mathbb{R}$, we have

$$
\limsup _{n \rightarrow+\infty} \int u^{2}\left(t+t_{n}, x+\rho\left(t_{n}\right)\right) \varphi\left(x-\rho\left(t_{n}+t\right)+\rho\left(t_{n}\right)-y_{0}\right) d x \leq \frac{C}{y_{0}},
$$

and so by weak convergence

$$
\int \widetilde{u}^{2}(t, x) \varphi\left(x-\widetilde{\rho}(t)-y_{0}\right) d x \leq \frac{C}{y_{0}} .
$$

Second, from (4.5), for fixed $t \in \mathbb{R}$,

$$
\begin{aligned}
& \limsup _{n \rightarrow+\infty} \int u^{2}\left(t+t_{n}, x+\rho\left(t_{n}\right)\right) \times \\
& \times\left(\varphi\left(x-\rho\left(t_{n}+t\right)+\rho\left(t_{n}\right)+\frac{t+t_{n}}{10}\right)-\varphi\left(x-\rho\left(t_{n}+t\right)+\rho\left(t_{n}\right)+y_{0}\right)\right) d x \leq \frac{C}{y_{0}} .
\end{aligned}
$$

Note that for fixed $t, y_{0}$, we have

$$
\begin{aligned}
\lim _{n \rightarrow+\infty} \varphi\left(x-\rho\left(t_{n}+t\right)+\rho\left(t_{n}\right)\right. & \left.+\frac{t+t_{n}}{10}\right)-\varphi\left(x-\rho\left(t_{n}+t\right)+\rho\left(t_{n}\right)+y_{0}\right) \\
& =\pi-\varphi\left(x-\widetilde{\rho}(t)+y_{0}\right)=\varphi\left(-x+\widetilde{\rho}(t)-y_{0}\right) .
\end{aligned}
$$

Thus, we obtain

$$
\int \widetilde{u}^{2}(t, x) \varphi\left(-x+\widetilde{\rho}(t)-y_{0}\right) d x \leq \frac{C}{y_{0}} .
$$

Finally, from (4.4)-(4.7), for any $y_{0}>1$, we have

$$
\lim _{n \rightarrow+\infty}\left|\int u^{2}\left(t_{n}, x\right)\left(\varphi\left(x-\rho\left(t_{n}\right)+y_{0}\right)-\varphi\left(x-\rho\left(t_{n}\right)-y_{0}\right)\right) d x-c^{+} \pi \int Q^{2}\right| \leq \frac{C}{y_{0}} .
$$

Thus, by $L_{l o c}^{2}$ convergence, for any $y_{0}>1$,

$$
\left|\int \widetilde{u}_{0}^{2}(x)\left(\varphi\left(x+y_{0}\right)-\varphi\left(x-y_{0}\right)\right) d x-c^{+} \pi \int Q^{2}\right| \leq \frac{C}{y_{0}} .
$$

Passing to the limit $y_{0} \rightarrow+\infty$, we obtain

$$
\left\|\widetilde{u}_{0}\right\|_{L^{2}}=\|\widetilde{u}(t)\|_{L^{2}}=\sqrt{c^{+}}\|Q\|_{L^{2}}=\left\|Q_{c^{+}}\right\|_{L^{2}} .
$$


4. Conclusion by Theorem 2. From Theorem 2, it follows that for some $c_{1}$ close to $c^{+}$and $x_{1}$ close to 0 , we have

$$
\widetilde{u}(t, x) \equiv Q_{c_{1}}\left(x-x_{1}-c_{1} t\right) .
$$

But $\|\widetilde{u}(t)\|_{L^{2}}=\left\|Q_{c^{+}}\right\|_{L^{2}}$ implies that $c_{1}=c^{+}$. Moreover, $\widetilde{\rho}(0)=0$ and $\widetilde{u}(0)=Q_{c^{+}}\left(x-x_{1}\right)=Q_{c^{+}}(x)+\widetilde{\eta}(0, x)$ where $x_{1}$ is small and $\int \widetilde{\eta}(0) Q_{c^{+}}^{\prime}=0$ imply $x_{1}=0$. In conclusion, $\widetilde{u}_{0}=Q_{c^{+}}$.

By a standard argument and (4.4), (4.7), we have obtained

$$
\begin{gathered}
u(t, .+\rho(t)) \rightarrow Q_{c^{+}} \quad \text { in } H^{\frac{1}{2}} \text { weak as } t \rightarrow+\infty, \\
\lim _{t \rightarrow+\infty} \int_{x>\frac{t}{10}}\left|u(t, x)-Q_{c^{+}}(x-\rho(t))\right|^{2} d x=0 .
\end{gathered}
$$

Thus Theorem 1 is a consequence of Theorem 2 .

\subsection{Proof of Theorem 2}

First, we note that it is sufficient to prove Theorem 2 in the case $\int u_{0}^{2}=\int Q^{2}$. Indeed, for $u_{0}$ satisfying the assumptions of Theorem 2 , set

$$
c_{1}=\frac{\int u_{0}^{2}}{\int Q^{2}} \quad \text { and } \quad \widetilde{u}(t)=\frac{1}{c_{1}} u\left(\frac{1}{c_{1}^{2}} t, \frac{1}{c_{1}} x\right) .
$$

Then, $\left|c_{1}-1\right| \leq C \alpha_{0}$ and $\widetilde{u}$ satisfies (1.1), $\int \widetilde{u}^{2}=\int Q^{2}$ and $\left\|\widetilde{u}_{0}-Q\right\|_{H^{\frac{1}{2}}} \leq$ $C \alpha_{0}$. Thus, by the stability property -see Introduction- for all $t$, there exists $y(t)$ such that $\sup _{t}\|\widetilde{u}(t)-Q(.-y(t))\|_{H^{\frac{1}{2}}} \leq C^{\prime} \alpha_{0}$. Moreover, $\widetilde{u}(t)$ also satisfies (1.9). If we prove $\widetilde{u}(t, x)=Q\left(x-t-x_{0}\right)$, with $\left|x_{0}\right| \leq C \alpha_{0}$, the result follows for $u(t)$.

The proof of Theorem 2 is by contradiction. Assume that there exists a sequence $u_{n}(t)$ of $H^{\frac{1}{2}}$ solutions of (1.1) such that

$$
\begin{aligned}
& \sup _{t \in \mathbb{R}}\left\|u_{n}(t)-Q\left(.-\rho_{n}(t)\right)\right\|_{L^{2}} \rightarrow 0 \quad \text { as } n \rightarrow+\infty, \\
& \int u_{n}^{2}(0)=\int Q^{2}, \quad \eta_{n} \not \equiv 0, \\
& \forall n, \forall \varepsilon>0, \exists A_{n, \varepsilon}>0 \text {, s.t. } \forall t \in \mathbb{R}, \int_{|x|>A_{n, \varepsilon}} u_{n}^{2}\left(t, x+\rho_{n}(t)\right) d x<\varepsilon,
\end{aligned}
$$

where $\rho_{n}(t)$ and $\eta_{n}(t)$ are defined from $u_{n}(t)$ by Lemma 1 . Note that $\int u_{n}^{2}(0)=\int Q^{2}$ implies

$$
\forall n, \forall t, \quad \int \eta_{n}^{2}(t)=-2 \int \eta_{n}(t) Q
$$


Define

$$
0 \not \equiv b_{n}=\sup _{t}\left\|\eta_{n}(t)\right\|_{L^{2}} \rightarrow 0 \quad \text { as } n \rightarrow+\infty \text {. }
$$

Then, there exists $t_{n}$ such that $\left\|\eta_{n}\left(t_{n}\right)\right\|_{L^{2}} \geq \frac{1}{2} b_{n}$. We set

$$
w_{n}(t, x)=\frac{\eta_{n}\left(t_{n}+t, x\right)}{b_{n}} .
$$

For such a sequence $w_{n}$, we claim the following result.

Proposition 5. (Weak convergence of renormalized solutions). There exists a subsequence of $\left(w_{n}\right)$ denoted by $\left(w_{n^{\prime}}\right)$ and $w \in C\left(\mathbb{R}, L^{2}(\mathbb{R})\right) \cap L^{\infty}\left(\mathbb{R}, L^{2}(\mathbb{R})\right)$ such that

$\forall t \in \mathbb{R}, \quad w_{n^{\prime}}(t) \rightarrow w(t) \quad$ in $L^{2}$ weak as $n \rightarrow+\infty$.

Moreover, $w(t)$ satisfies for some continuous function $\beta(t)$ :

$$
\begin{aligned}
& w_{t}=(\mathcal{L} w)_{x}+\beta(t) Q^{\prime} \quad \text { on } \mathbb{R} \times \mathbb{R}, \\
& w(0) \neq 0, \quad \int w Q=\int w Q^{\prime}=0, \\
& \forall t \in \mathbb{R}, \forall x_{0}>1, \quad \int_{|x|>x_{0}} w^{2}(t, x) d x \leq \frac{C}{x_{0}} .
\end{aligned}
$$

Proposition 5 is in contradiction with Theorem 3. Thus, for $\alpha_{0}>0$ small, for $u(t)$ satisfying the assumptions of Theorem 2 , we have $\eta \equiv 0$ so that $\rho^{\prime}(t)=1$ (by Lemma 1 ) and $u(t, x)=Q\left(x-\rho(0)-t\right.$ ), with $|\rho(0)| \leq C \alpha_{0}$.

Therefore, we are reduced to prove Proposition 5.

Proof of Proposition 5. One can actually prove a strong $L^{2}$ convergence result. See the end of the proof.

Note that the main point in Proposition 5 is the fact that $w \neq 0$. This requires a strong convergence in $L^{2}$ for some suitable $t$.

Decay estimate. From Proposition 2, we have

$$
\begin{aligned}
& \int \eta_{n}^{2}\left(t_{0}, x\right)\left(\varphi\left(x-x_{0}\right)-\varphi\left(-x_{0}\right)\right) d x \\
& \leq \int \eta_{n}^{2}(t, x)\left(\varphi\left(x-x_{0}-\lambda\left(t_{0}-t\right)\right)-\varphi\left(-x_{0}-\lambda\left(t_{0}-t\right)\right)\right) d x+\frac{C b_{n}^{2}}{x_{0}} .
\end{aligned}
$$

Letting $t \rightarrow-\infty$ and using (4.12), we obtain, for any $x_{0}>1$,

$$
\int \eta_{n}^{2}\left(t_{0}, x\right)\left(\varphi\left(x-x_{0}\right)-\varphi\left(-x_{0}\right)\right) d x \leq \frac{C b_{n}^{2}}{x_{0}} .
$$


Similarly, arguing on $\eta_{n}(-t,-x)$, for any $x_{0}>1$,

$$
\int \eta_{n}^{2}\left(t_{0}, x\right)\left(\varphi\left(x_{0}\right)-\varphi\left(x+x_{0}\right)\right) d x \leq \frac{C b_{n}^{2}}{x_{0}}
$$

which gives, by (3.23), similarly as in the proof of (3.24):

(4.15) $\forall x_{0}>1, \quad \int_{|x|>x_{0}} \eta_{n}^{2}(t, x) d x \leq \frac{C b_{n}^{2}}{x_{0}} \quad$ and $\quad \int_{|x|>x_{0}} w_{n}^{2}(t, x) d x \leq \frac{C}{x_{0}}$.

Local smoothing estimate on $w_{n}$. Let $\varphi$ be defined in (2.6) for a fixed value of $A$ ( $A=1$ for example). Then,

$$
\int_{0}^{1} \int\left|D^{\frac{1}{2}}\left(w_{n}(t, x) \sqrt{\varphi^{\prime}(x)}\right)\right|^{2} d x d t \leq C .
$$

Proof of (4.16). First, we claim the following estimate:

$$
\frac{1}{2} \frac{d}{d t} \int \eta_{n}^{2} \varphi \leq-\frac{1}{2} \int\left|D^{\frac{1}{2}}\left(\eta_{n} \sqrt{\varphi^{\prime}}\right)\right|^{2}+C \int \eta_{n}^{2} \leq-\frac{1}{2} \int\left|D^{\frac{1}{2}}\left(\eta_{n} \sqrt{\varphi^{\prime}}\right)\right|^{2}+C b_{n}^{2} .
$$

Thus, by integration,

$$
\begin{gathered}
\forall t \in \mathbb{R}, \quad \int_{t}^{t+1} \int\left|D^{\frac{1}{2}}\left(\eta_{n} \sqrt{\varphi^{\prime}}\right)\right|^{2} d x d t \leq C b_{n}^{2} \quad \text { and } \\
\\
\int_{t}^{t+1} \int\left|D^{\frac{1}{2}}\left(w_{n} \sqrt{\varphi^{\prime}}\right)\right|^{2} d x d t \leq C .
\end{gathered}
$$

Now, we justify (4.17). Using direct computations, Lemma 3, (2.2) and then $\left|\int \eta_{n}^{3} \varphi^{\prime}\right| \leq C \int|\eta|^{3} \leq C \int \eta^{2}$ (by (A.1)), we get

$$
\begin{aligned}
\frac{1}{2} \frac{d}{d t} \int \eta_{n}^{2} \varphi \\
=-\int\left(\mathcal{L} \eta_{n}-\frac{1}{2} \eta_{n}^{2}\right)\left(\eta_{n x} \varphi+\eta_{n} \varphi^{\prime}\right)+\left(\rho_{n}^{\prime}-1\right) \int Q^{\prime} \eta_{n} \varphi-\frac{1}{2}\left(\rho_{n}^{\prime}-1\right) \int \eta_{n}^{2} \varphi^{\prime} \\
=\int\left(\left(\mathcal{H} \eta_{n x}\right) \eta_{n x} \varphi+\left(\mathcal{H} \eta_{n x}\right) \eta_{n} \varphi^{\prime}\right)-\frac{1}{2} \int \eta_{n}^{2} \varphi^{\prime}+\frac{1}{2} \int \eta_{n}^{2}\left(-Q^{\prime} \varphi+Q \varphi^{\prime}\right) \\
\quad+\frac{1}{3} \int \eta_{n}^{3} \varphi^{\prime}+\left(\rho_{n}^{\prime}-1\right) \int Q^{\prime} \eta_{n} \varphi-\frac{1}{2}\left(\rho_{n}^{\prime}-1\right) \int \eta_{n}^{2} \varphi^{\prime} \\
\leq \int\left(\mathcal{H} \eta_{n x}\right) \eta_{n} \varphi^{\prime}+C \int \eta_{n}^{2} .
\end{aligned}
$$


Using (A.3) and then (A.1), we have

$$
\begin{aligned}
-\int(\mathcal{H} & \left.\eta_{n x}\right) \eta_{n} \varphi^{\prime}=\int \eta_{n} D\left(\eta_{n} \varphi^{\prime}\right) \\
& =\int \eta_{n} \sqrt{\varphi^{\prime}} D\left(\eta_{n} \sqrt{\varphi^{\prime}}\right)+\int \eta_{n}\left(D\left(\eta_{n} \varphi^{\prime}\right)-\sqrt{\varphi^{\prime}} D\left(\eta_{n} \sqrt{\varphi^{\prime}}\right)\right) \\
& \geq \int\left|D^{\frac{1}{2}}\left(\eta_{n} \sqrt{\varphi^{\prime}}\right)\right|^{2}-C\left\|\eta_{n}\right\|_{L^{2}}\left\|D\left(\eta_{n} \varphi^{\prime}\right)-\sqrt{\varphi^{\prime}} D\left(\eta_{n} \sqrt{\varphi^{\prime}}\right)\right\|_{L^{2}} \\
& \geq \int\left|D^{\frac{1}{2}}\left(\eta_{n} \sqrt{\varphi^{\prime}}\right)\right|^{2}-C\left\|\eta_{n}\right\|_{L^{2}}\left\|\eta_{n} \sqrt{\varphi^{\prime}}\right\|_{L^{4}}\left\|D \sqrt{\varphi^{\prime}}\right\|_{L^{4}} \\
& \geq \frac{1}{2} \int\left|D^{\frac{1}{2}}\left(\eta_{n} \sqrt{\varphi^{\prime}}\right)\right|^{2}-C\left\|\eta_{n}\right\|_{L^{2}}^{2} .
\end{aligned}
$$

(Note that we have used $\left\|D \sqrt{\varphi^{\prime}}\right\|_{L^{4}}<+\infty$.) Thus (4.17) is proved.

Compactness in $L^{2}$ for some time. From the equation of $\eta_{n}$ and (2.2), it follows that

$$
\frac{d}{d t} \int \eta_{n}^{2}=-\frac{1}{2} \int Q^{\prime} \eta_{n}^{2}+\left(\rho_{n}^{\prime}-1\right) \int Q^{\prime} \eta_{n} \quad \text { and so } \quad\left|\frac{d}{d t} \int \eta_{n}^{2}\right| \leq C_{0} \int \eta_{n}^{2} .
$$

In particular, by the definition of $t_{n}, \forall t \in[0,1], \int \eta_{n}^{2}\left(t+t_{n}\right) \geq e^{-C_{0}} b_{n}^{2}$ and so

$$
\forall t \in[0,1], \quad\left\|w_{n}(t)\right\|_{L^{2}} \geq e^{-\frac{1}{2} C_{0}}=\delta>0 .
$$

It follows from (4.16) that for all $n$, there exists $\tau_{n} \in[0,1]$ such that $\int\left|D^{\frac{1}{2}}\left(w_{n}\left(\tau_{n}\right) \sqrt{\varphi^{\prime}}\right)\right|^{2} \leq C$. Thus, there exists a subsequence of $\left(w_{n}\right)$ (still denoted by $\left.\left(w_{n}\right)\right)$ and $s_{0} \in[0,1], W \in H^{\frac{1}{2}}$ such that

$$
w_{n}\left(\tau_{n}\right) \sqrt{\varphi^{\prime}} \rightarrow W \quad \text { in } H^{\frac{1}{2}} \text { weak, } \quad \tau_{n} \rightarrow s_{0} \quad \text { as } n \rightarrow+\infty .
$$

But (by possibly extracting a further subsequence), there exists $w_{s_{0}} \in L^{2}$ such that

$$
\tau_{n} \rightarrow s_{0}, \quad w_{n}\left(\tau_{n}\right) \rightarrow w_{s_{0}} \quad \text { in } L^{2} \text { weak as } n \rightarrow+\infty .
$$

It follows that $W=w_{s_{0}} \sqrt{\varphi^{\prime}}$. Since $\sqrt{\varphi^{\prime}}>0$ on $\mathbb{R}$, we get

$$
w_{n}\left(\tau_{n}\right) \rightarrow w_{s_{0}} \quad \text { in } L_{l o c}^{2} \text { as } n \rightarrow+\infty .
$$

By (4.15) and (4.20), we finally get

$$
w_{n}\left(\tau_{n}\right) \rightarrow w_{s_{0}} \quad \text { in } L^{2} \text { as } n \rightarrow+\infty, \quad \int w_{s_{0}} Q^{\prime}=0, \quad w_{s_{0}} \neq 0 .
$$

Note also that from (4.13) and $\int \eta_{n} Q^{\prime}=0$, we have

$$
\int w_{s_{0}} Q=\int w_{s_{0}} Q^{\prime}=0
$$


Weak convergence for all time. Consider $\widetilde{w}(t) \in C\left(\mathbb{R}, L^{2}(\mathbb{R})\right)$ the unique solution of

$$
\widetilde{w}_{t}=(\mathcal{L} \widetilde{w})_{x} \quad \text { on } \mathbb{R} \times \mathbb{R}, \quad \widetilde{w}\left(s_{0}\right)=w_{s_{0}}, \quad \text { on } \mathbb{R} .
$$

(It is clear by a standard energy estimate and regularization arguments that the corresponding Cauchy problem is well-posed in $L^{2}$ ).

Now, to obtain weak convergence, we need to remove some terms from the equation of $w_{n}$, following some arguments in [16], Lemma 8 and beginning of proof of Lemma 11. We write

$$
\begin{aligned}
w_{n t} & =\left(\mathcal{L} w_{n}-\frac{b_{n}}{2} w_{n}^{2}\right)_{x}+\frac{1}{b_{n}}\left(\rho_{n}^{\prime}-1\right)\left(Q+b_{n} w_{n}\right)_{x} \\
& =\left(\mathcal{L} w_{n}\right)_{x}-\frac{b_{n}}{2}\left(w_{n}^{2}\right)_{x}+\beta_{n} Q^{\prime}+b_{n} F_{n}^{\prime}+b_{n} \widetilde{\beta}_{n}\left(w_{n}\right)_{x}
\end{aligned}
$$

where

$$
\beta_{n}=\frac{1}{\int\left(Q^{\prime}\right)^{2}} \int w_{n} \mathcal{L}\left(Q^{\prime \prime}\right), \quad \widetilde{\beta}_{n}=\frac{1}{b_{n}}\left(\rho_{n}^{\prime}-1\right), \quad F_{n}=\frac{1}{b_{n}}\left(\widetilde{\beta}_{n}-\beta_{n}\right) Q .
$$

Set $\widetilde{w}_{n}(t)=w_{n}(t)-Q^{\prime} \int_{\tau_{n}}^{t} \beta_{n}(s) d s$. Then, the equation of $\widetilde{w}_{n}(t)$ writes

$$
\widetilde{w}_{n t}=\left(\mathcal{L} \widetilde{w}_{n}\right)_{x}-\frac{b_{n}}{2}\left(w_{n}^{2}\right)_{x}+b_{n} F_{n}^{\prime}+b_{n} \widetilde{\beta}_{n}\left(\widetilde{w}_{n}\right)_{x}+b_{n} \widetilde{\beta}_{n} Q^{\prime \prime} \int_{\tau_{n}}^{t} \beta_{n}(s) d s .
$$

We claim the following weak convergence result.

Lemma 9. For all $t \in \mathbb{R}$,

$$
\widetilde{w}_{n}(t) \rightarrow \widetilde{w}(t) \quad \text { in } L^{2} \text { weak. }
$$

Assuming this lemma, from (2.5), we have, for all $t$,

$$
\widetilde{\beta}_{n}(t) \rightarrow \widetilde{\beta}(t)=\frac{1}{\int\left(Q^{\prime}\right)^{2}} \int \widetilde{w} \mathcal{L}\left(Q^{\prime \prime}\right), \quad \int_{\tau_{n}}^{t} \widetilde{\beta}_{n}(s) d s \rightarrow \int_{s_{0}}^{t} \widetilde{\beta}(s) d s .
$$

Set $w(t)=\widetilde{w}(t)+Q^{\prime} \int_{s_{0}}^{t} \widetilde{\beta}(s) d s$. Then, $w(t)$ solves

$$
w_{t}=(\mathcal{L} w)_{x}+\widetilde{\beta} Q^{\prime},
$$

and $w\left(s_{0}\right)=w_{s_{0}} \neq 0$. Moreover, for all $t \in \mathbb{R}$,

$$
w_{n}(t) \rightarrow w(t) \quad \text { in } L^{2} \text { weak. }
$$

Finally, from (4.13) and $\int \eta_{n} Q^{\prime}=0$, we have $\int w(t) Q=\int w(t) Q^{\prime}=0$, and by weak convergence and (4.15), we have

$$
\forall x_{0}>1, \forall t, \quad \int_{|x|>x_{0}} w^{2}(t, x) d x \leq \frac{C}{x_{0}} .
$$

Thus, we are reduced to prove Lemma 9. 
Proof of Lemma 9. Set

$$
G_{1, n}=-\frac{1}{2} w_{n}^{2}, \quad G_{2, n}=F_{n}+\widetilde{\beta}_{n} \widetilde{w}_{n}+\widetilde{\beta}_{n} Q^{\prime} \int_{\tau_{n}}^{t} \beta_{n}(s) d s, \quad G_{n}=G_{1, n}+G_{2, n} .
$$

Observe that

$$
\left\|G_{1, n}\right\|_{L^{1}}+\left\|G_{2, n}\right\|_{L^{2}} \leq C(t), \quad \text { with } C(t) \text { bounded on bounded intervals. }
$$

Let $T \in \mathbb{R}$. By $\sup _{t}\left\|w_{n}(t)\right\|_{L^{2}} \leq C$ and the expression of $\widetilde{w}_{n}$, we have $\sup _{[-T, T]}\left\|\widetilde{w}_{n}(t)\right\|_{L^{2}} \leq C_{T}$.

Let $g \in C_{0}^{\infty}(\mathbb{R})$ and let $v$ solve the problem

$$
\left\{\begin{array}{l}
\partial_{t} v=\mathcal{L}\left(v_{x}\right) \\
v_{\mid t=T}=g
\end{array}\right.
$$

Then

$$
\begin{aligned}
\int\left(\widetilde{w}_{n}\right. & -\widetilde{w})(T) g(x) d x-\int\left(w_{n}\left(\tau_{n}\right)-w\left(\tau_{n}\right)\right) v\left(\tau_{n}\right) d x \\
& =\int_{\tau_{n}}^{T} \int \partial_{t}\left(\left(\widetilde{w}_{n}-\widetilde{w}\right)(t) v(t, x)\right) d x d t \\
& =\int_{\tau_{n}}^{T} \int\left(\left(\mathcal{L} \widetilde{w}_{n}\right)_{x}-(\mathcal{L} \widetilde{w})_{x}+b_{n}\left(G_{n}\right)_{x}\right) v(t, x)+\left(\widetilde{w}_{n}-\widetilde{w}\right)(\mathcal{L} v)_{x} d x d t \\
& =-b_{n} \int_{\tau_{n}}^{T} \int G_{n} v_{x}(t, x) d x d t
\end{aligned}
$$

The energy method gives

$$
\|v\|_{L^{\infty}\left(\left[\tau_{n}, T\right], L^{2}(\mathbb{R})\right)}+\left\|v_{x}\right\|_{L^{\infty}\left(\left[\tau_{n}, T\right] \times \mathbb{R}\right)}+\left\|v_{x}\right\|_{L^{\infty}\left(\left[\tau_{n}, T\right], L^{2}(\mathbb{R})\right)} \leq C .
$$

Moreover, by continuity of $t \mapsto w(t)$ in $L^{2}$,

$$
\begin{aligned}
& \lim _{n \rightarrow+\infty} \int\left(w_{n}\left(\tau_{n}\right)-w\left(\tau_{n}\right)\right) v\left(\tau_{n}\right) d x \\
& =\lim _{n \rightarrow+\infty} \int\left(w_{n}\left(\tau_{n}\right)-w\left(s_{0}\right)\right) v\left(\tau_{n}\right) d x+\lim _{n \rightarrow+\infty} \int\left(w\left(s_{0}\right)-w\left(\tau_{n}\right)\right) v\left(\tau_{n}\right) d x=0 .
\end{aligned}
$$

Thus,

$$
\widetilde{w}_{n}(T) \rightarrow \widetilde{w}(T) \quad \text { as } n \rightarrow+\infty .
$$

and the proof of Lemma 9 is concluded. 
Alternate proof by strong $L^{2}$ convergence for all time. Now, we use Theorem 6 in Section 6 to prove strong $L^{2}$ convergence of the sequence $\left(w_{n}(t)\right)$ for all $t$.

Let $T>0$. Set

$$
\begin{aligned}
\zeta_{n}(t, x)=w_{n}( & \left.t, x-\rho_{n}(t)+\rho_{n}(0)\right) \\
+ & \frac{1}{b_{n}}\left[Q\left(x-\left(\rho_{n}(t)-\rho_{n}(0)\right)\right)-Q(x-t)\right],
\end{aligned}
$$

so that

$$
\begin{aligned}
u_{n}\left(t, x+\rho_{n}(0)\right) & =Q\left(x-\rho_{n}(t)+\rho_{n}(0)\right)+b_{n} w_{n}\left(t, x-\rho_{n}(t)+\rho_{n}(0)\right) \\
& =Q(x-t)+b_{n} \zeta_{n}(t, x)
\end{aligned}
$$

and $\zeta_{n}$ satisfies

$$
\begin{gathered}
\left(\zeta_{n}\right)_{t}=\left(-\mathcal{H}\left(\zeta_{n}\right)_{x}-Q(x-t) \zeta_{n}\right)_{x}-\frac{b_{n}}{2}\left(\zeta_{n}^{2}\right)_{x}, \\
\left\|\zeta_{n}(t)\right\|_{L^{2}} \leq C_{T}, \quad \forall t \in[-T, T] .
\end{gathered}
$$

Indeed, since $\left|\rho_{n}^{\prime}(t)-1\right| \leq C\left\|\eta_{n}\right\|_{L^{2}} \leq C b_{n}$, we have

$$
\left|\rho_{n}(t)-\rho_{n}(0)-t\right| \leq C b_{n}|t|,
$$

and the estimate on $\zeta_{n}$ follows.

On the one hand, Theorem 6 applied to $\frac{1}{C_{T}} \zeta_{n}$ for $n$ large enough (so that $b_{n}$ is small enough) implies that $t \in[-T, T] \mapsto \zeta_{n}(t) \in L^{2}$ is equicontinuous in $n$.

On the other hand, from (4.16), we have

$$
\int_{[-T, T]} \int\left|D^{\frac{1}{2}}\left(\zeta_{n}(t, x) \sqrt{\varphi^{\prime}(x)}\right)\right|^{2} d x d t \leq C_{T}
$$

and the decay property (4.15) also holds for $\zeta(t)$ on $[-T, T]$ with constant depending on $T$. In particular, there exists $N \subset[-T, T]$ of zero Lebesgue measure such that for all $t \in[-T, T] \backslash N, \int\left|D^{\frac{1}{2}}\left(\zeta_{n}(t, x) \sqrt{\varphi^{\prime}(x)}\right)\right|^{2} d x d t<+\infty$. Now, we choose a dense and countable subset $I$ of $[-T, T]$ such that for all $t \in I, \int\left|D^{\frac{1}{2}}\left(\zeta_{n}(t, x) \sqrt{\varphi^{\prime}(x)}\right)\right|^{2} d x d t<+\infty$. Arguing as in the proof of (4.21), and using a diagonal argument, there exists a subsequence of $\left(\zeta_{n}\right)$ which we will still denote by $\left(\zeta_{n}\right)$ such that for any $t \in I, \zeta_{n}(t) \rightarrow \zeta(t)$ in $L^{2}$ strong as $n \rightarrow+\infty$. Using the equicontinuity, we obtain

$$
\forall t \in[-T, T], \quad \zeta_{n}(t) \rightarrow \zeta(t) \quad \text { in } L^{2} \text { strong as } n \rightarrow+\infty .
$$


By (4.24) and $\left|\rho_{n}^{\prime}-1\right| \leq C b_{n}$, we may also assume that for the same subsequence

$$
\forall t \in[-T, T], \quad \frac{1}{b_{n}}\left(\rho_{n}(t)-\rho_{n}(0)-t\right) \rightarrow \kappa(t) .
$$

Now, we deduce from (4.23), (4.25) and (4.26) that $\forall t \in[-T, T], w_{n}(t) \rightarrow w(t)=\eta(t, .+t)+\kappa(t) Q^{\prime}$ in $L^{2}$ strong as $n \rightarrow+\infty$.

\subsection{Proof of Remark 1}

Let $u(t)$ be a solution satisfying the assumptions of Theorem 1 . Let $c^{+}, \rho(t)$ and $\eta(t)$ be defined as in the proof of Theorem 1. In particular, by (4.9), we have

$$
\lim _{t \rightarrow+\infty} \int_{x>\frac{t}{10}-\rho(t)}|\eta(t, x)|^{2} d x=0 .
$$

To prove (1.11), we use the identity (2.34) on $\eta$, where $\varphi=\frac{\pi}{2}+\arctan \left(\frac{x}{A}\right)$, $A>1$ large enough be to defined later:

$$
\begin{aligned}
\frac{1}{2} \frac{d}{d t} \int \eta^{2} \varphi= & \int\left(\mathcal{H} \eta_{x}\right) \eta \varphi^{\prime}+\int\left(\mathcal{H} \eta_{x}\right) \eta_{x} \varphi-\frac{1}{2} \int \eta^{2} \varphi^{\prime}+\frac{1}{2} \int \eta^{2}\left(-Q^{\prime} \varphi+Q \varphi^{\prime}\right) \\
& +\frac{1}{3} \int \eta^{3} \varphi^{\prime}+\left(\rho^{\prime}-1\right) \int Q^{\prime} \eta \varphi-\frac{1}{2}\left(\rho^{\prime}-1\right) \int \eta^{2} \varphi^{\prime} .
\end{aligned}
$$

We claim that for $A$ large enough and $\alpha_{0}$ small enough, for $C>0$ independent of $A$,

$$
\frac{1}{2} \frac{d}{d t} \int \eta^{2} \varphi \leq \int\left(\mathcal{H} \eta_{x}\right) \eta \varphi^{\prime}+C \int \frac{\eta^{2}}{1+x^{2}}
$$

Indeed, by Lemma 3, we have $\int\left(\mathcal{H} \eta_{x}\right) \eta_{x} \varphi \leq \frac{C}{A} \int \eta^{2} \varphi^{\prime}$. By the definition of $Q$, $\int \eta^{2}\left(-Q^{\prime} \varphi+Q \varphi^{\prime}\right) \leq C \int \frac{\eta^{2}}{1+x^{2}}$. By (2.30) (note that the constant in (2.30) is independent of $A)\left|\int \eta^{3} \varphi^{\prime}\right| \leq C \alpha_{0} \int \eta^{2} \varphi^{\prime}$. Finally, the last two terms are controlled using (2.2), so that (4.28) is proved for $A$ large enough, $\alpha_{0}$ small enough.

Now, we use (4.19) on $\eta$. We obtain

$$
\begin{gathered}
\frac{1}{2} \frac{d}{d t} \int \eta^{2} \varphi \\
(4.29) \leq-\int\left|D^{\frac{1}{2}}\left(\eta \sqrt{\varphi^{\prime}}\right)\right|^{2}+C\|\eta\|_{L^{2}}\left\|\eta \sqrt{\varphi^{\prime}}\right\|_{L^{4}}\left\|D \sqrt{\varphi^{\prime}}\right\|_{L^{4}}+C \int \frac{\eta^{2}}{1+x^{2}}
\end{gathered}
$$


Note that for $A>1$, we have $\frac{1}{1+x^{2}} \leq C \varphi$ on $\mathbb{R}$. Let $t_{0}>0$. Integrating the above estimate on $\left[t_{0}, t_{0}+1\right]$, we get

$$
\int_{t_{0}}^{t_{0}+1} \int\left|D^{\frac{1}{2}}\left(\eta \sqrt{\varphi^{\prime}}\right)\right|^{2} \leq C \sup _{t \in\left[t_{0}, t_{0}+1\right]}\left(\int \eta^{2}(t) \varphi+C\left\|\eta \sqrt{\varphi^{\prime}}\right\|_{L^{4}}\left\|D \sqrt{\varphi^{\prime}}\right\|_{L^{4}}\right) .
$$

On the other hand, by (A.3), we have

$$
\begin{aligned}
\int\left|D^{\frac{1}{2}} \eta\right|^{2} \varphi^{\prime} & \leq 2 \int\left|D^{\frac{1}{2}}\left(\eta \sqrt{\varphi^{\prime}}\right)\right|^{2}+2 \int\left|\left(D^{\frac{1}{2}} \eta\right) \sqrt{\varphi^{\prime}}-D^{\frac{1}{2}}\left(\eta \sqrt{\varphi^{\prime}}\right)\right|^{2} \\
& \leq 2 \int\left|D^{\frac{1}{2}}\left(\eta \sqrt{\varphi^{\prime}}\right)\right|^{2}+C\|\eta\|_{L^{4}}^{2}\left\|D^{\frac{1}{2}} \sqrt{\varphi^{\prime}}\right\|_{L^{4}}^{2} \\
& \leq 2 \int\left|D^{\frac{1}{2}}\left(\eta \sqrt{\varphi^{\prime}}\right)\right|^{2}+C\left\|D^{\frac{1}{2}} \sqrt{\varphi^{\prime}}\right\|_{L^{4}}^{2}
\end{aligned}
$$

Thus, we obtain

$$
\begin{aligned}
\int_{t_{0}}^{t_{0}+1} \int\left|D^{\frac{1}{2}} \eta\right|^{2} \varphi^{\prime} d t & \leq C \sup _{t \in\left[t_{0}, t_{0}+1\right]}\left(\int \eta^{2}(t) \varphi+C\left\|\eta \sqrt{\varphi^{\prime}}\right\|_{L^{4}}\left\|D \sqrt{\varphi^{\prime}}\right\|_{L^{4}}\right) \\
& +C\left\|D^{\frac{1}{2}} \sqrt{\varphi^{\prime}}\right\|_{L^{4}}^{2}
\end{aligned}
$$

We have $\left\|D^{\frac{1}{2}} \sqrt{\varphi^{\prime}}\right\|_{L^{4}}^{2} \leq C A^{-\frac{3}{2}},\left\|D \sqrt{\varphi^{\prime}}\right\|_{L^{4}} \leq C A^{-\frac{5}{4}}$ and

$$
\left\|\eta \sqrt{\varphi^{\prime}}\right\|_{L^{4}} \leq\|\eta\|_{L^{8}}\left\|\sqrt{\varphi^{\prime}}\right\|_{L^{8}} \leq C A^{-\frac{3}{8}}
$$

Therefore,

$$
\int_{t_{0}}^{t_{0}+1} \int\left|D^{\frac{1}{2}} \eta(t, x)\right|^{2} \frac{d x d t}{1+\left(\frac{x}{A}\right)^{2}} \leq A \sup _{t \in\left[t_{0}, t_{0}+1\right]}\left(\int \eta^{2}(t) \varphi\right)+C A^{-\frac{1}{2}} .
$$

We now choose $A$ depending on $t_{0}$ :

$$
A=A_{t_{0}}=\min \left(\frac{\sqrt{t_{0}}}{2},\left(\sup _{t \in\left[t_{0}, t_{0}+1\right]} \int_{x \geq \frac{t}{10}-\rho(t)} \eta^{2}(t, x) d x\right)^{-\frac{1}{2}}\right) .
$$

For this choice of $A_{t_{0}}$, we have $\lim _{t_{0} \rightarrow+\infty} A_{t_{0}}=+\infty$ and, since $\frac{t}{10}-\rho(t) \leq-\frac{t}{2}$,

$$
A \sup _{t \in\left[t_{0}, t_{0}+1\right]}\left(\int \eta^{2}(t) \varphi\right) \leq C A \sup _{t \in\left[t_{0}, t_{0}+1\right]}\left(\int_{x \geq \frac{t}{10}-\rho(t)} \eta^{2}(t)\right)+\frac{C A}{t_{0}} \leq C A^{-1} .
$$

so that $\lim _{t_{0} \rightarrow+\infty} A \sup _{t \in\left[t_{0}, t_{0}+1\right]}\left(\int \eta^{2}(t) \varphi\right)=0$. It follows that

$$
\lim _{t_{0} \rightarrow+\infty} \int_{t_{0}}^{t_{0}+1} \int\left|D^{\frac{1}{2}} \eta(t, x)\right|^{2} \frac{d x d t}{1+x^{2}}=0 .
$$




\section{Multi-soliton case}

Using the previous arguments and the strategy of [21] for the gKdV equation, we obtain the following result concerning multi-soliton solutions of (1.1).

Theorem 4 (Asymptotic stability of a sum of decoupled solitons). Let $N \geq 1$ and $0<c_{1}^{0}<\ldots<c_{N}^{0}$. There exist $L_{0}>0, A_{0}>0$ and $\alpha_{0}>0$ such that if $u_{0} \in H^{\frac{1}{2}}$ satisfies for some $0 \leq \alpha<\alpha_{0}, L \geq L_{0}$,

$$
\left\|u_{0}-\sum_{j=1}^{N} Q_{c_{j}^{0}}\left(.-y_{j}^{0}\right)\right\|_{H^{\frac{1}{2}}} \leq \alpha \quad \text { where } \forall j \in\{2, \ldots, N\}, y_{j}^{0}-y_{j-1}^{0} \geq L,
$$

and if $u(t)$ is the solution of (1.1) corresponding to $u(0)=u_{0}$, then there exist $\rho_{1}(t), \ldots, \rho_{N}(t)$ such that the following hold

(a) Stability of the sum of $N$ decoupled solitons.

$$
\forall t \geq 0, \quad\left\|u(t)-\sum_{j=1}^{N} Q_{c_{j}^{0}}\left(x-\rho_{j}(t)\right)\right\|_{H^{\frac{1}{2}}} \leq A_{0}\left(\alpha+\frac{1}{L}\right) .
$$

(b) Asymptotic stability of the sum of $N$ solitons. There exist $c_{1}^{+}, \ldots, c_{N}^{+}$, with $\left|c_{j}^{+}-c_{j}^{0}\right| \leq A_{0}\left(\alpha+\frac{1}{L}\right)$, such that

$$
\forall j, \quad u\left(t, .+\rho_{j}(t)\right) \rightarrow Q_{c_{j}^{+}} \quad \text { in } H^{\frac{1}{2}} \text { weak as } t \rightarrow+\infty,
$$

$$
\left\|u(t)-\sum_{j=1}^{N} Q_{c_{j}^{+}}\left(.-\rho_{j}(t)\right)\right\|_{L^{2}\left(x \geq \frac{1}{10} c_{1}^{0} t\right)}^{\rightarrow} \rightarrow 0, \quad \rho_{j}^{\prime}(t) \rightarrow c_{j}^{+} \text {as } t \rightarrow+\infty .
$$

Recall that the Benjamin-Ono equation admits explicit multi-soliton solution. We denote by $U_{N}\left(x ; c_{j}, y_{j}\right)$ the explicit family of $N$-soliton profiles, see e.g. [22, formula (1.7)] and Appendix A (see also references in [22]). We obtain the following corollary of the above Theorem and the continuous dependence of the solution in $H^{\frac{1}{2}}$.

Let $N \geq 1,0<c_{1}^{0}<\cdots<c_{N}^{0}$ and set

$$
d_{N}(u)=\inf \left\{\left\|u-U_{N}\left(. ; c_{j}^{0}, y_{j}\right)\right\|_{H^{\frac{1}{2}}}, y_{j} \in \mathbb{R}\right\} .
$$

Corollary 1. (Asymptotic stability in $H^{\frac{1}{2}}$ of multi-solitons). For all $\delta>0$, there exists $\alpha>0$ such that if $d_{N}\left(u_{0}\right) \leq \alpha$ then for all $t \in \mathbb{R}, d_{N}(u(t)) \leq \delta$.

Recall that a result of stability in $H^{1}$ of double solitons for the BO equation was proved by variational methods in [23]. See also [22] for stability related results. 


\subsection{Sketch of the stability argument [31]}

For the reader's convenience, we now sketch the proof of the stability argument for one soliton (see statement in the Introduction). Let $u(t)$ be an $H^{\frac{1}{2}}$ solution of (1.1) such that $u(0)$ is close to $Q$ in $H^{\frac{1}{2}}$. Let $c^{+}>0$ be close to 1 such that $\int u^{2}(0)=c^{+} \int Q^{2}$. We use Lemma 1 on $u(t)$ around $Q_{c^{+}}$ so that $\eta(t, x)=u(t, x+\rho(t))-Q_{c^{+}}(x)$ satisfies $\int \eta(t) Q_{c^{+}}^{\prime}=0$ and by $L^{2}$ conservation $\int \eta(t) Q_{c^{+}}=-\frac{1}{2} \int \eta^{2}(t)$.

We define the functional

$$
\mathcal{G}(u(t))=E(u(t))+c^{+} \int u^{2}(t) .
$$

Observing that $\mathcal{G}(u(t))=\mathcal{G}(u(0))$ and so expanding $u(t)$ in $\mathcal{G}(u(t))$, we obtain

$$
\left(\mathcal{L}_{c^{+}} \eta(t), \eta(t)\right)+O\left(\eta^{3}(t)\right)=\left(\mathcal{L}_{c^{+}} \eta(0), \eta(0)\right)+O\left(\eta^{3}(0)\right)
$$

where $\mathcal{L}_{c^{+}} \eta=-\mathcal{H} \eta_{x}+c^{+} \eta-Q_{c^{+}} \eta$. By the positivity property of $\mathcal{L}_{c^{+}}$, (property (A.10) of $\mathcal{L}$ and a scaling argument), we then obtain

$$
\|\eta(t)\|_{H^{\frac{1}{2}}} \leq C\|\eta(0)\|_{H^{\frac{1}{2}}}
$$

Note that $\left|\int \eta Q_{c^{+}}\right| \leq C\|\eta\|_{L^{2}}^{2}$ replaces the orthogonality condition $\int \eta Q_{c^{+}}=0$.

\subsection{Sketch of the proof of Theorem 4}

The proof is the same as the proof of Theorem 1 in [21].

First, we recall four lemmas (corresponding to Lemmas 1-4 in [21]) which are the main tools in proving Theorem 4.

Lemma 10. (Decomposition of the solution). There exist $L_{1}, \alpha_{1}, K_{1}>0$ such that the following is true. If for $L>L_{1}, 0<\alpha<\alpha_{1}, t_{0}>0$,

$$
\sup _{0 \leq t \leq t_{0}}\left(\inf _{y_{j}>y_{j-1}+L}\left\{\left\|u(t, .)-\sum_{j=1}^{N} Q_{c_{j}^{0}}\left(.-y_{j}\right)\right\|_{H^{\frac{1}{2}}}\right\}\right)<\alpha,
$$

then there exist unique $C^{1}$ functions $c_{j}:\left[0, t_{0}\right] \rightarrow(0,+\infty), \rho_{j}:\left[0, t_{0}\right] \rightarrow \mathbb{R}$, such that

$$
\begin{gathered}
\eta(t, x)=u(t, x)-R(t, x) \\
\text { where } R(t, x)=\sum_{j=1}^{N} R_{j}(t, x), \quad R_{j}(t, x)=Q_{c_{j}(t)}\left(x-\rho_{j}(t)\right),
\end{gathered}
$$

satisfies the following orthogonality conditions

$$
\forall j, \forall t \in\left[0, t_{0}\right], \quad \int R_{j}(t) \eta(t)=\int\left(R_{j}(t)\right)_{x} \eta(t)=0 .
$$


Moreover, there exists $C>0$ such that $\forall t \in\left[0, t_{0}\right]$,

$$
\begin{gathered}
\|\eta(t)\|_{H^{\frac{1}{2}}}+\sum_{j=1}^{N}\left|c_{j}(t)-c_{j}^{0}\right| \leq C \alpha \\
\forall j,\left|c_{j}^{\prime}(t)\right|+\left|\rho_{j}^{\prime}(t)-c_{j}(t)\right| \leq C\left(\|\eta(t)\|_{L^{2}}+\frac{1}{L}\right) .
\end{gathered}
$$

Remark 5. In the rest of the argument, the modulation in the scaling parameter for all time (i.e. the introduction of $c_{j}(t)$ ) is not necessary. Indeed, modulation at $t=0$ would be sufficient since we deal with the subcritical case. However, we have preferred to introduce this modulation to match the strategy of [21].

Expanding $u(t)$ in the energy conservation and using $E\left(Q_{c}\right)=c^{2} E(Q)$, we have

Lemma 11. There exists $C>0$ such that in the context of Lemma 10, $\forall t \in\left[0, t_{0}\right]$

$$
\begin{gathered}
\left.\mid E(Q) \sum_{j=1}^{N}\left[c_{j}^{2}(t)\right)-c_{j}^{2}(0)\right]+\frac{1}{2} \int\left(\eta_{x} \mathcal{H} \eta-R \eta^{2}\right)(t) \mid \\
\leq C\left(\|\eta(0)\|_{H^{\frac{1}{2}}}^{2}+\|\eta(t)\|_{H^{\frac{1}{2}}}^{3}+\frac{1}{L}\right) .
\end{gathered}
$$

We consider $\varphi$ defined as in (2.6), with $A$ large enough, and we set $\forall j \in\{2, \ldots, N\}$,

$$
\mathcal{I}_{j}(t)=\int u^{2}(t, x) \varphi\left(x-m_{j}(t)\right) d x, \quad m_{j}(t)=\frac{1}{2}\left(\rho_{j-1}(t)+\rho_{j}(t)\right) .
$$

Then, proceeding as in the proof of Proposition 1, we obtain the following.

Lemma 12. There exists $C>0$ such that in the context of Lemma 10,

$$
\forall j \in\{2, \ldots, N\}, \forall t \in\left[0, t_{0}\right], \quad \mathcal{I}_{j}(t)-\mathcal{I}_{j}(0) \leq \frac{C}{L} .
$$

Finally, setting $c(t, x)=c_{1}(t)+\sum_{j=2}^{N}\left(c_{j}(t)-c_{j-1}(t)\right) \varphi\left(x-m_{j}(t)\right)$, and proceeding as in the proof of Propositions 3 and 4 , we have

Lemma 13. There exists $\lambda>0$ such that in the context of Lemma 10,

$$
\forall t \in\left[0, t_{0}\right], \quad \mathcal{G}_{N}(t):=\int \eta_{x} \mathcal{H} \eta+c(t, x) \eta^{2}-Q \eta^{2} \geq \lambda\|\eta(t)\|_{H^{\frac{1}{2}}}^{2} .
$$


Recall that the introduction of the functional $\mathcal{G}_{N}(t)$ for the problem of stability of multi-soliton solutions is justified as follows. For the stability of one soliton, the suitable functional is $\mathcal{G}(u(t))$ defined in $(5.5)$. For the case of $N$ solitons, we introduce the functional $\mathcal{G}_{N}(t)$ which is approximately $E(u(t))+c_{j}(0) \int u^{2}(t)$ around the soliton $Q_{c_{j}}$. Then, we observe (using the energy conservation and Lemma 11) that this quantity is almost decreasing. This is sufficient to conclude the stability argument for several solitons. We now sketch the argument. We refer to [21], Section 3 for more details in the stability proof.

Sketch of the proof of the stability. Let

$$
\mathcal{V}_{A_{0}}(L, \alpha)=\left\{u \in H^{\frac{1}{2}} ; \inf _{y_{j}-y_{j-1} \geq L}\left\|u-\sum_{j=1}^{N} Q_{c_{j}^{0}}\left(.-y_{j}\right)\right\|_{H^{\frac{1}{2}}} \leq A_{0}\left(\alpha+\frac{1}{L}\right)\right\} \text {. }
$$

Part (a) of Theorem 4 is a consequence of the following proposition and continuity arguments.

Proposition 6. There exist $A_{0}>0, L_{0}>0$ and $\alpha_{0}>0$ such that, for all $u_{0} \in H^{\frac{1}{2}}$, if

$$
\left\|u_{0}-\sum_{j=1}^{N} Q_{c_{j}^{0}}\left(.-y_{j}^{0}\right)\right\|_{H^{\frac{1}{2}}} \leq \alpha,
$$

where $L \geq L_{0}, 0<\alpha<\alpha_{0}, y_{j}^{0}-y_{j-1}^{0}+L$, and if for $t^{*}>0$,

$$
\forall t \in\left[0, T^{*}\right], \quad u(t) \in \mathcal{V}_{A_{0}}(L, \alpha),
$$

where $u(t)$ is the solution of (1.1), then

$$
\forall t \in\left[0, T^{*}\right], \quad u(t) \in \mathcal{V}_{\frac{1}{2} A_{0}}(L, \alpha)
$$

The proof of Proposition 6 is exactly the same as the proof of Proposition 1 in [21], using Lemmas 10-13. In particular, we first prove

$$
\forall t \in\left[0, t^{*}\right], \quad \sum_{j=1}^{N}\left|c_{j}(t)-c_{j}(0)\right| \leq C_{1}\left(\|\eta(t)\|_{H^{\frac{1}{2}}}^{2}+\|\eta(0)\|_{H^{\frac{1}{2}}}^{2}+\frac{1}{L}\right),
$$

and then

$$
\|\eta(t)\|_{H^{\frac{1}{2}}}^{2} \leq C_{2}\left(\|\eta(0)\|_{H^{\frac{1}{2}}}^{2}+\frac{1}{L}\right)
$$

where $C_{1}, C_{2}>0$ are independent of $A_{0}$, and we then conclude by using the decomposition of $u(t)$ is terms of $\eta(t)$ and $R(t)$. 
Note that in proving (5.6), we make use of the following algebraic fact:

$$
E\left(Q_{c}\right)=c^{2} E(Q), \quad \int Q_{c}^{2}=c \int Q^{2}, \quad E(Q)=-\frac{1}{2} \int Q^{2} .
$$

The last formula is easily obtained from the equation of $Q$ multiplying by $Q$ and then by $x Q^{\prime}$ and using $\int\left(\mathcal{H} Q^{\prime}\right)\left(x Q^{\prime}\right)=0$. This allows us to prove the following estimate

$$
\begin{gathered}
\left|E(Q) \sum_{j=1}^{N}\left(c_{j}(t)-c_{j}(0)\right)+\int Q^{2} \sum_{j=1}^{N}\left\{c_{j}(0)\left(c_{j}(t)-c_{j}(0)\right)\right\}\right| \\
\leq C \sum_{j=1}^{N}\left|c_{j}(t)-c_{j}(0)\right|^{2}
\end{gathered}
$$

which is the analogue of (44) in [21].

The proof of part (b) of Theorem 4 is exactly the same as in [21], Section 4, using Theorem 2, the monotonicity arguments (Proposition 1) and Theorem 5. It follows closely the proof of Theorem 1 in the present paper.

The proof of Corollary 1 is omitted since it is the same as the proof of Corollary 1 in [21].

\section{Weak convergence and well-posedness results}

\subsection{Weak convergence}

Theorem 5. (Weak continuity of the BO flow map). Let $\left(u_{n}\right)$ be a sequence of global $H^{\frac{1}{2}}$ solutions of equation (1.1). Assume that $u_{n}(0) \rightarrow u_{0}$ in $H^{\frac{1}{2}}$ weak and let $u(t)$ be the solution of (1.1) corresponding to $u(0)=u_{0}$. Then, for all $t \in \mathbb{R}, u_{n}(t) \rightarrow u(t)$ in $H^{\frac{1}{2}}$ weak.

Proof of Theorem 5. Let $u_{0, n}=u_{n}(0)$. It is sufficient to prove the result for $T \in[0,1]$.

Step 1. $H^{2}$ case. Here, we assume $u_{0, n} \rightarrow u_{0}$ in $H^{2}$. Let $w_{n}=u_{n}-u$. The equation for $w_{n}$ is

$$
\left\{\begin{array}{l}
w_{n t}+\mathcal{H}\left(w_{n}\right)_{x x}+u_{n} w_{n x}+u_{x} w_{n}=0 \\
w_{n}(0)=\psi_{n}, \quad \psi_{n}=u_{0, n}-u_{0} .
\end{array}\right.
$$

Fix $t=T, g \in C_{0}^{\infty}(\mathbb{R})$. For a function $\widetilde{u}$ to be determined, we consider the solution $v(t)$ of

$$
\left\{\begin{array}{l}
v_{t}+\mathcal{H} v_{x x}+(\widetilde{u} v)_{x}-u_{x} v=0, \\
v(T)=g
\end{array}\right.
$$


Then

$$
\begin{aligned}
\int w_{n}(T, x) g(x) d x-\int \psi_{n}(x) v(0, x) d x & =\int_{0}^{T} \int w_{n t}(t) v(t)+\int_{0}^{T} \int w_{n}(t) v_{t} \\
& =\mathbf{I}+\mathbf{I I} .
\end{aligned}
$$

$\mathbf{I}=\int_{0}^{T} \int w_{n}\left(\mathcal{H} v_{x x}+\left(u_{n} v\right)_{x}-u_{x} v\right), \quad \mathbf{I I}=-\int_{0}^{T} \int w_{n}\left(\mathcal{H} v_{x x}+(\widetilde{u} v)_{x}-u_{x} v\right)$ so that

$$
\begin{aligned}
\int w_{n}(T, x) g(x) d x-\int \psi_{n}(x) v(0, x) d x & =\int_{0}^{T} \int w_{n}\left(\left(u_{n}-\widetilde{u}\right) v\right)_{x} \\
& =-\int_{0}^{T} \int w_{n x}\left(u_{n}-\widetilde{u}\right) v .
\end{aligned}
$$

We can assume, after passing to a subsequence, that $u_{n}-\widetilde{u} \rightarrow 0$ in $L_{\text {loc }}^{2}(\mathbb{R} \times$ $[0, T])$. Next, we will show that given $\varepsilon>0$, there exists $R>0$ such that

$$
\left|\int_{0}^{T} \int_{|x|>R} w_{n x}\left(u_{n}-\widetilde{u}\right) v\right| \leq \varepsilon, \quad \text { uniformly in } n .
$$

In fact, since $\left\|w_{n x}\right\|_{L^{\infty}} \leq C, \sup _{t}\|v\|_{L^{2}} \leq C$ and $\sup _{t}\left\|u_{n}-\widetilde{u}\right\|_{L^{2}} \leq C$, the claim is clear.

But then, $\mathbf{I}+\mathbf{I I} \rightarrow 0$ as $n \rightarrow+\infty$. We only needed $\sup _{t}\|v\|_{L^{2}} \leq C$, which needs $\widetilde{u}_{x} \in L^{\infty}, u_{x} \in L^{\infty}$, which are both clear. (We use the energy method to bound $v$.)

Step 2. General case. Fix $N$ large and define $u_{0, n}^{N}$ such that $\widehat{u_{0, n}^{N}}(\xi)=$ $\mathbf{1}_{[-N, N]}(\xi) \widehat{u}_{0, n}(\xi)$, where $\mathbf{1}_{I}$ is the characteristic function of $I$. Note that

$$
\left\|u_{0, n}^{N}-u_{0, n}\right\|_{L^{2}}^{2}=\int_{|\xi| \geq N}\left|\widehat{u_{0, n}^{N}}(\xi)\right|^{2} \leq \frac{1}{N}\left\|u_{0, n}\right\|_{H^{\frac{1}{2}}}^{2} \leq \frac{C}{N},
$$

so that $u_{0, n}^{N} \rightarrow u_{0, n}$ in $L^{2}$ as $N \rightarrow+\infty$, uniformly in $n$.

Fix $g \in C_{0}^{\infty}, T \in \mathbb{R}, \varepsilon>0$. The proof of the $L^{2}$ continuity of the flow map (see [11]) shows that

$$
\begin{gathered}
\sup _{t \in[0,1]}\left\|u^{N}(t)-u(t)\right\|_{L^{2}} \leq C\left\|u_{0}^{N}-u_{0}\right\|_{L^{2}}, \\
\sup _{t \in[0,1]}\left\|u_{n}^{N}(t)-u_{n}(t)\right\|_{L^{2}} \leq C\left\|u_{0, n}^{N}-u_{0, n}\right\|_{L^{2}}
\end{gathered}
$$

for some universal constant $C>0$. We fix $N$ such that

$$
\left|\int\left(u_{n}(T)-u(T)\right) g-\int\left(u_{n}^{N}(T)-u^{N}(T)\right) g\right| \leq \frac{\varepsilon}{2}, \quad \text { uniformly in } n .
$$

But, for fixed $N$, we let $n \rightarrow+\infty$, and use step 1 and the proof is concluded. 


\subsection{Well-posedness result for the nonlinear $\mathrm{BO}$ equation with potential}

In this subsection, for $0<b<b_{0}, b_{0}$ small, we consider the IVP

$$
\left\{\begin{array}{l}
v_{t}=\left(-\mathcal{H} v_{x}\right)_{x}-(Q(x-t) v)_{x}-\frac{b}{2}\left(v^{2}\right)_{x}=0 \quad \text { on }[-T, T] \times \mathbb{R}, \\
v(t=0, x)=v_{0}(x) \quad \text { on } \mathbb{R} .
\end{array}\right.
$$

The well-posedness of the Cauchy problem in $L^{2}$ for this equation is clear from [11] since $u(t, x)=Q(x-t)+b v(t, x)$ satisfies the BO equation. Our main concern is a result of equicontinuity of the map $t \mapsto v(t)$ in $L^{2}$ with respect to $b$. To establish such a result we follow the strategy of [11] on equation (6.2), using the special form of $Q$ and keeping track of the dependency in $b$.

Theorem 6. (a) Let $v_{0} \in H^{\infty}$. Then, there exists $T=T(Q)>0$ and a unique solution $v=S_{b}^{\infty}\left(v_{0}\right)$ of $(6.2)$ in $[-T, T], v \in C\left([-T, T], H^{\infty}\right)$.

(b) There exists a constant $C$, independent of b such that

$$
\sup _{t \in[-T, T]}\|v(t)\|_{H^{2}} \leq C\left\|v_{0}\right\|_{H^{2}} .
$$

(c) The mapping $S_{b}^{\infty}$ extends uniquely to a continuous mapping $S_{b}^{0}: L^{2} \rightarrow$ $C\left([-T, T], L^{2}\right)$, and there exists $C$, independent of $b$ such that

$$
\sup _{t \in[-T, T]}\|v(t)\|_{L^{2}} \leq C\left\|v_{0}\right\|_{L^{2}} .
$$

Moreover, given $v_{0} \in L^{2},\left\|v_{0}\right\|_{L^{2}} \leq 2$, for any $\varepsilon>0$, there exits $\delta=\delta\left(v_{0}, \varepsilon\right)>0$ ( $\delta$ independent of b) such that for any $v_{1} \in L^{2}$, $\left\|v_{1}\right\|_{L^{2}} \leq 2$,

$$
\left\|v_{0}-v_{1}\right\|_{L^{2}} \leq \delta \quad \Rightarrow \quad \sup _{t \in[-T, T]}\left\|S_{b}^{0}\left(v_{0}\right)(t)-S_{b}^{0}\left(v_{1}\right)(t)\right\|_{L^{2}} \leq \varepsilon
$$

Finally, there exists $\widetilde{\delta}=\widetilde{\delta}\left(v_{0}, \varepsilon\right)>0$ (independent of $b$ ) such that for any $t, t^{\prime} \in[-T, T]$,

$$
\left|t-t^{\prime}\right| \leq \widetilde{\delta} \quad \Rightarrow \quad\left\|S_{b}^{0}\left(v_{0}\right)(t)-S_{b}^{0}\left(v_{0}\right)\left(t^{\prime}\right)\right\|_{L^{2}} \leq \varepsilon .
$$

Reduction of the proof. For $0<\lambda \ll 1$, consider $v_{\lambda}(t, x)=\lambda v\left(\lambda^{2} t, \lambda x\right)$. Then $v_{\lambda}$ solves

$$
\left\{\begin{array}{l}
\left(v_{\lambda}\right)_{t}=\left(-\mathcal{H}\left(v_{\lambda}\right)_{x}\right)_{x}-\left(\lambda Q\left(\lambda x-\lambda^{2} t\right) v_{\lambda}\right)_{x}-\frac{b}{2}\left(v_{\lambda}^{2}\right)_{x}=0 \text { on }[-T, T] \times \mathbb{R} \\
v_{\lambda}(t=0, x)=v_{0, \lambda}(x) \text { on } \mathbb{R}, \quad v_{0, \lambda}(x)=\lambda v_{0}(\lambda x)
\end{array}\right.
$$

Define

$$
Q_{\lambda}(t, x)=\lambda Q\left(\lambda x-\lambda^{2} t\right)
$$


Then the proof of Theorem 6 reduces to prove that for the following (IVP)

$$
\left\{\begin{array}{l}
v_{t}=\left(-\mathcal{H} v_{x}\right)_{x}-\left(Q_{\lambda}(t, x) v\right)_{x}-\frac{b}{2}\left(v^{2}\right)_{x}=0 \quad \text { on }[-1,1] \times \mathbb{R}, \\
v(t=0, x)=v_{0}(x) \quad \text { on } \mathbb{R}, \quad\left\|v_{0}\right\|_{L^{2}} \leq \lambda^{\frac{1}{2}}
\end{array}\right.
$$

we have

Theorem 7. There exists $b_{0}, \lambda>0$ small enough such that if $0<b<b_{0}$, the following hold

(a) Assume $v_{0} \in H^{\infty}$. Then, there exists a unique solution $v=S_{b}^{\infty}\left(v_{0}\right)$ of (6.8) in $[-1,1], v \in C\left([-1,1], H^{\infty}\right)$.

(b) There exists a constant $C$, independent of $b$ such that

$$
\sup _{t \in[-1,1]}\|v(t)\|_{H^{2}} \leq C\left\|v_{0}\right\|_{H^{2}}
$$

(c) The mapping $S_{b}^{\infty}$ extends uniquely to a continuous mapping $S_{b}^{0}: L^{2} \rightarrow$ $C\left([-1,1], L^{2}\right)$, and there exists $C$, independent of $b$ such that

$$
\sup _{t \in[-1,1]}\|v(t)\|_{L^{2}} \leq C\left\|v_{0}\right\|_{L^{2}}
$$

Moreover, given $v_{0} \in L^{2},\left\|v_{0}\right\|_{L^{2}} \leq \lambda^{\frac{1}{2}}$, for any $\varepsilon>0$, there exits $\delta=\delta\left(v_{0}, \varepsilon\right)>0$ ( $\delta$ independent of $\left.b\right)$ such that for any $v_{1} \in L^{2}$, $\left\|v_{1}\right\|_{L^{2}} \leq \lambda^{\frac{1}{2}}$

$$
\left\|v_{0}-v_{1}\right\|_{L^{2}} \leq \delta \Rightarrow \sup _{t \in[-1,1]}\left\|S_{b}^{0}\left(v_{0}\right)(t)-S_{b}^{0}\left(v_{1}\right)(t)\right\|_{L^{2}} \leq \varepsilon
$$

Finally, there exists $\widetilde{\delta}=\widetilde{\delta}\left(v_{0}, \varepsilon\right)>0$ (independent of $b$ ) such that for any $t, t^{\prime} \in[-1,1]$,

$$
\left|t-t^{\prime}\right| \leq \widetilde{\delta} \quad \Rightarrow \quad\left\|S_{b}^{0}\left(v_{0}\right)(t)-S_{b}^{0}\left(v_{0}\right)\left(t^{\prime}\right)\right\|_{L^{2}} \leq \varepsilon
$$

The proof of Theorem 7 is based on the following three propositions.

Proposition 7. Assume $v_{0} \in H^{\infty}$, then there exists $T=T\left(\left\|v_{0}\right\|_{H^{2}}\right)$ and a unique solution $v$ of $(6.8)$ in $(-T, T)$. Also, for any $\sigma \geq 2$,

$$
\sup _{t \in(-T, T)}\|u(t)\|_{H^{\sigma}} \leq C\left(\sigma,\left\|v_{0}\right\|_{\sigma}, \sup _{t \in(-T, T)}\|v(t)\|_{H^{2}}\right) .
$$

In particular, the constant $C$ is independent of $b,\left(0<b<b_{0}\right)$ and $\lambda<1$. 
Proposition 7 is a consequence of the energy method, taking into account that

$$
\left\|\partial_{x} Q_{\lambda}\right\|_{L^{1}\left((-1,1), L_{x}^{\infty}\right)} \leq C
$$

Proposition 8. For $\lambda$ small enough, we have that if $T \in(0,1],\left\|v_{0}\right\|_{L^{2}} \leq \lambda^{\frac{1}{2}}$, $v=S^{\infty}\left(v_{0}\right) \in C\left((-T, T), H^{\infty}\right)$ is a solution, then

$$
\sup _{t \in[-T, T]}\|v(t)\|_{H^{2}} \leq C\left\|v_{0}\right\|_{H^{2}}
$$

where $C$ is independent of $b\left(0<b<b_{0}\right)$.

Proposition 9. For $v_{0} \in H^{\infty}, N \in[1, \infty)$, $\left\|v_{0}\right\|_{L^{2}} \leq \lambda^{\frac{1}{2}}$, let $\widehat{v_{0}^{N}}(\xi)=$ $\mathbf{1}_{[-N, N]}(\xi) \hat{v}_{0}(\xi), v_{0}^{N} \in H^{\infty}$. Then,

$$
\begin{gathered}
\sup _{t \in(-1,1)}\left\|S_{b}^{\infty}\left(v_{0}\right)(t)-S_{b}^{\infty}\left(v_{0}^{N}\right)(t)\right\|_{L^{2}} \leq C\left\|v_{0}-v_{0}^{N}\right\|_{L^{2}}, \\
\sup _{t \in(-1,1)}\left\|S_{b}^{\infty}\left(v_{0}\right)(t)\right\|_{L^{2}} \leq C\left\|v_{0}\right\|_{L^{2} .}
\end{gathered}
$$

where $C$ is independent of $b\left(0<b<b_{0}\right)$.

Proof of Theorem 7 from Propositions 7, 8 and 9. First, note that Propositions 7 and 8 clearly give (a) and (b) in Theorem 7. Let us turn to the proof of (c): it suffices to show first that if $v_{0, n} \in H^{\infty}, \lim _{n \rightarrow+\infty} v_{0, n}=v_{0}$ in $L^{2}$, the sequence $S_{b}^{\infty}\left(v_{0, n}\right)$ is Cauchy in $C\left([-1,1], L^{2}\right)$. Let $\varepsilon>0$ be given. We want to show that there exists $M_{\varepsilon}$ (independent of $b$ ) such that

$$
m, n \geq M_{\varepsilon} \Rightarrow \sup _{t \in[-1,1]}\left\|S_{b}^{\infty}\left(v_{0, n}\right)(t)-S_{b}^{\infty}\left(v_{0, m}\right)(t)\right\|_{L^{2}} \leq \varepsilon
$$

Observe that

$$
\left\|v_{0, n}-v_{0, n}^{N}\right\|_{L^{2}} \leq\left\|v_{0}-v_{0}^{N}\right\|_{L^{2}}+\left\|v_{0}-v_{0, n}\right\|_{L^{2}}
$$

Hence, we can fix $N=N\left(\varepsilon, v_{0}\right)$ large and $M_{\varepsilon}^{1}$ large such that $\left\|v_{0, n}-v_{0, n}^{N}\right\|_{L^{2}} \leq$ $\frac{\varepsilon}{4 C}$, for $n \geq M_{\varepsilon}^{1}$, where $C$ is the constant in Proposition $9\left(\left\|v_{0, n}\right\|_{L^{2}} \leq \lambda^{\frac{1}{2}}\right)$. Then, by Proposition 9, for $n \geq M_{\varepsilon}^{1}$,

$$
\sup _{t \in[-1,1]}\left\|S_{b}^{\infty}\left(v_{0, n}\right)(t)-S_{b}^{\infty}\left(v_{0, n}^{N}\right)(t)\right\|_{L^{2}} \leq \frac{\varepsilon}{4}
$$


It remains to estimate $\sup _{t \in[-1,1]}\left\|S_{b}^{\infty}\left(v_{0, n}^{N}\right)(t)-S_{b}^{\infty}\left(v_{0, m}^{N}\right)(t)\right\|_{L^{2}}$. But energy estimates for the difference equation give

$$
\begin{aligned}
& \sup _{t \in[-1,1]}\left\|S_{b}^{\infty}\left(v_{0, n}^{N}\right)(t)-S_{b}^{\infty}\left(v_{0, m}^{N}\right)(t)\right\|_{L^{2}} \\
& \leq\left\|v_{0, n}^{N}-v_{0, m}^{N}\right\|_{L^{2}} \exp \left(C \int_{-1}^{1}\left\|\partial_{x}\left(S_{b}^{\infty}\left(v_{0, n}^{N}\right)\right)(t)\right\|_{L_{x}^{\infty}}+C\left\|\partial_{x}\left(S_{b}^{\infty}\left(v_{0, m}^{N}\right)\right)(t)\right\|_{L_{x}^{\infty}}\right) \\
& \leq\left\|v_{0, n}-v_{0, m}\right\|_{L^{2}} \exp \left(C \sup _{|t|<1}\left\|S_{b}^{\infty}\left(v_{0, n}^{N}\right)(t)\right\|_{H^{2}}+C \sup _{|t|<1}\left\|S_{b}^{\infty}\left(v_{0, m}^{N}\right)(t)\right\|_{H^{2}}\right) \\
& \leq\left\|v_{0, n}-v_{0, m}\right\|_{L^{2}} \exp \left(C N^{2}\left\|v_{0, n}\right\|_{L^{2}}+C N^{2}\left\|v_{0, n}\right\|_{L^{2}}\right) \\
& \leq\left\|v_{0, n}-v_{0, m}\right\|_{L^{2}} \exp \left(C N^{2}\right) \leq \frac{\varepsilon}{2},
\end{aligned}
$$

for $n, m$ large (we have used the estimate of Proposition 8). Also, by Proposition 9, we have $\sup _{t \in(-1,1)}\left\|S_{b}^{\infty}\left(v_{0, n}\right)(t)\right\|_{L^{2}} \leq C$. Thus, we obtain the unique extension $S_{b}^{0}$ and (6.10) holds.

To check (6.11), fix $v_{0},\left\|v_{0}\right\|_{L^{2}} \leq \lambda^{\frac{1}{2}}$, let $\varepsilon>0$ be given. With $C$ as in Proposition 9, find $N\left(N=N\left(\varepsilon, v_{0}\right)\right)$ so large that $\left\|v_{0}-v_{0}^{N}\right\|_{L^{2}} \leq \frac{\varepsilon}{8 C}$. Now find $\delta_{1}=\delta_{1}\left(\varepsilon, v_{0}\right)$ so small that if $\left\|v_{0}-v_{1}\right\|_{L^{2}} \leq \delta_{1}$, then $\left\|v_{1}-v_{1}^{N}\right\|_{L^{2}} \leq \frac{\varepsilon}{4 C}$. We have

$$
\begin{aligned}
& \sup _{t \in[-1,1]}\left\|S_{b}^{0}\left(v_{0}\right)(t)-S_{b}^{0}\left(v_{1}\right)(t)\right\|_{L^{2}} \leq \sup _{t \in[-1,1]}\left\|S_{b}^{0}\left(v_{0}\right)(t)-S_{b}^{0}\left(v_{0}^{N}\right)(t)\right\|_{L^{2}} \\
& \quad+\sup _{t \in[-1,1]}\left\|S_{b}^{0}\left(v_{1}\right)(t)-S_{b}^{0}\left(v_{1}^{N}\right)(t)\right\|_{L^{2}}+\sup _{t \in[-1,1]}\left\|S_{b}^{0}\left(v_{1}^{N}\right)(t)-S_{b}^{0}\left(v_{0}^{N}\right)(t)\right\|_{L^{2}} .
\end{aligned}
$$

By Proposition 9, the first two terms are smaller than $\frac{\varepsilon}{2}$. For the last one, we again use the energy estimate and get, as before

$$
\sup _{t \in[-1,1]}\left\|S_{b}^{0}\left(v_{1}^{N}\right)(t)-S_{b}^{0}\left(v_{0}^{N}\right)(t)\right\|_{L^{2}} \leq C\left\|v_{1}-v_{0}\right\|_{L^{2}} \exp \left(C N^{2}\right)
$$

using Propositions 8 and 9 and (6.11) follows.

For (6.12), first find $N=N\left(\varepsilon, v_{0}\right)$ so large that $\left\|v_{0}-v_{0}^{N}\right\|_{L^{2}} \leq \frac{\varepsilon}{4 C}$, where $C$ is as in Proposition 9. Then,

$$
\sup _{t \in[-1,1]}\left\|S_{b}^{0}\left(v_{0}\right)(t)-S_{b}^{0}\left(v_{0}^{N}\right)(t)\right\|_{L^{2}} \leq \frac{\varepsilon}{4}
$$

and we are reduced to showing, for $N$ fixed that if $\left|t-t^{\prime}\right| \leq \widetilde{\delta}$, then $\left\|S_{b}^{0}\left(v_{0}^{N}\right)(t)-S_{b}^{0}\left(v_{0}^{N}\right)\left(t^{\prime}\right)\right\|_{L^{2}} \leq \frac{\varepsilon}{2}$. 
Let $f(t)=\left\|S_{b}^{0}\left(v_{0}^{N}\right)(t)\right\|_{L^{2}}^{2}$. The energy method, combined with Proposition 8 shows that $\left|f^{\prime}(t)\right| \leq f(0) \exp \left(C N^{2}\right)$. But then, for $\left|t-t^{\prime}\right| \leq \widetilde{\delta}_{1}$, $\left|f(t)-f\left(t^{\prime}\right)\right| \leq \frac{\varepsilon}{4}$. But

$$
\begin{aligned}
& \left\|S_{b}^{0}\left(v_{0}^{N}\right)(t)-S_{b}^{0}\left(v_{0}^{N}\right)\left(t^{\prime}\right)\right\|_{L^{2}}^{2}=f(t)+f\left(t^{\prime}\right)-2 \int S_{b}^{0}\left(v_{0}^{N}\right)(t) \cdot S_{b}^{0}\left(v_{0}^{N}\right)\left(t^{\prime}\right) d x \\
& =f\left(t^{\prime}\right)-f(t)+2 \int S_{b}^{0}\left(v_{0}^{N}\right)(t)\left[S_{b}^{0}\left(v_{0}^{N}\right)(t)-S_{b}^{0}\left(v_{0}^{N}\right)\left(t^{\prime}\right)\right] d x .
\end{aligned}
$$

Let $v^{N}(t)=S_{b}^{0}\left(v_{0}^{N}\right)(t)$. The second term equals

$$
\begin{aligned}
& 2 \int v^{N}(t) \int_{t^{\prime}}^{t} \partial_{s} v^{N}(s) d s d x \\
& =2 \int_{t^{\prime}}^{t} \int v_{N}(t)\left[-\mathcal{H} \partial_{x}^{2} v^{N}(s)-\left(Q_{\lambda} v^{N}\right)_{x}-\frac{b}{2}\left(\left(v^{N}\right)^{2}\right)_{x}(s)\right] d x d s .
\end{aligned}
$$

But by Proposition $8, \sup _{t \in[-1,1]}\left\|v^{N}(t)\right\|_{H^{2}} \leq C\left\|v_{0}^{N}\right\|_{H^{2}} \leq C N^{2}$. Thus, the second term is controlled by $C\left|t-t^{\prime}\right| N$, and the proof is complete, provided we prove Propositions 8 and 9 .

Proof of Propositions 8 and 9. Step 1. Assume $v_{0} \in H^{\infty},\left\|v_{0}\right\|_{H^{2}} \leq M$ and $0<T \leq 2, v=S_{b}^{\infty}(t)$ exists in $[-T, T]$. Then, there exist $\lambda_{0}=\lambda_{0}(M)$, $b_{0}=b_{0}(M)$ such that for $0<\lambda<\lambda_{0}, 0 \leq b<b_{0}$, we have

$$
\sup _{t \in[-T, T]}\|v(t)\|_{H^{2}} \leq 2\left\|v_{0}\right\|_{H^{2}}
$$

Proof of (6.14). Note that $\left\|\partial_{x}^{k} Q \lambda\right\|_{L^{\infty}} \leq C_{k} \lambda^{k+1}$. Let $f(t)=\|v(t)\|_{H^{2}}^{2}$. The standard energy method shows that

$$
\left|f^{\prime}(t)\right| \leq C\left(\lambda_{0}^{2}+b_{0}\left\|\partial_{x} v(t)\right\|_{L_{x}^{\infty}}\right) f(t) \leq\left(\lambda_{0}^{2}+b_{0}(f(t))^{\frac{1}{2}}\right) f(t) .
$$

Integrating the ODE gives the result.

As a corollary, we obtain under the circumstances of Step 1 that $v$ exists in $(-1,1)$ and

$$
\sup _{t \in[-2,2]}\|v(t)\|_{H^{2}} \leq 2\left\|v_{0}\right\|_{H^{2}} .
$$

Step 2. From now on, we will follow closely [11]. Some of the ideas used before were developed in a forthcoming paper [8]. We have now reduced everything to a priori estimates. We will change notation slightly to match [11]. We then study the problem

$$
\left\{\begin{array}{l}
u_{t}+\mathcal{H} u_{x x}+\left(Q_{\lambda} u\right)_{x}+b\left(\frac{1}{2} u^{2}\right)_{x}=0 \quad(t, x) \in(-1,1) \times \mathbb{R}, \\
u_{\mid t=0}=\phi, \quad\|\phi\|_{L^{2}} \leq \lambda^{\frac{1}{2}}
\end{array}\right.
$$


We use the notation $P_{\text {low }}, P_{ \pm \text {high }}$ as in [11]:

$$
\begin{gathered}
P_{\text {low }} \text { defined by the Fourier multiplier } \xi \rightarrow \mathbf{1}_{\left[-2^{10}, 2^{10}\right]}(\xi) ; \\
P_{ \pm \text {high }} \text { defined by the Fourier multiplier } \xi \rightarrow \mathbf{1}_{\left[2^{10}, \infty\right)}( \pm \xi) \\
P_{ \pm} \text {defined by the Fourier multiplier } \xi \rightarrow \mathbf{1}_{[0, \infty)}( \pm \xi)
\end{gathered}
$$

Let $\phi_{0}=P_{\text {low }} \phi \in H^{\infty}$, real-valued, $\left\|\phi_{0}\right\|_{H^{2}} \leq 2^{20}=M$. We choose $\lambda_{0}, b_{0}$ as in Step 1 and its corollary, so that Proposition 7 and these results gives, with $u_{0}^{(1)}=S_{b}^{\infty}\left(\phi_{0}\right)(t)$ that

$$
\sup _{t \in[-2,2]}\left\|\partial_{t}^{\sigma_{1}} \partial_{x}^{\sigma_{2}} u_{0}^{(1)}\right\|_{L_{x}^{2}} \leq C_{\sigma_{1}, \sigma_{2}}\|\phi\|_{L^{2}}, \quad \sigma_{i} \geq 0 .
$$

Let $\widetilde{u}=u-u_{0}^{(1)}$. The equation for $\widetilde{u}$ is

$$
\left\{\begin{array}{l}
\widetilde{u}_{t}+\mathcal{H} \widetilde{u}_{x x}+\left(Q_{\lambda} \widetilde{u}\right)_{x}+b\left(u_{0}^{(1)} \widetilde{u}\right)_{x}+b\left(\frac{1}{2} \widetilde{u}^{2}\right)_{x}=0, \\
\widetilde{u}_{\mid t=0}=P_{+\operatorname{high}} \phi+P_{-\operatorname{high}} \phi .
\end{array}\right.
$$

Let now $u_{0}(t, x)=Q_{\lambda}(t, x)+b u_{0}^{(1)}(t, x)$. Then

$$
\sup _{t \in[-2,2]}\left\|\partial_{t}^{\sigma_{1}} \partial_{x}^{\sigma_{2}} u_{0}\right\|_{L_{x}^{2}} \leq C_{\sigma_{1}, \sigma_{2}}\left(\lambda_{0}^{\frac{1}{2}}+b_{0}\right) .
$$

We now want to construct $U_{0}$ similarly to [11], with the following properties $\partial_{x} U_{0}(t, x)=\frac{1}{2} u_{0}(t, x), U_{0}(0,0)=0$ and

$$
\sup _{t \in[-2,2]}\left\|\partial_{t}^{\sigma_{1}} \partial_{x}^{\sigma_{2}} U_{0}(t, .)\right\|_{L_{x}^{2}} \leq C_{\sigma_{1}, \sigma_{2}}\left(\lambda_{0}^{\frac{1}{2}}+b_{0}\right)
$$

where $\sigma_{1}, \sigma_{2} \geq 0,\left(\sigma_{1}, \sigma_{2}\right) \neq(0,0)$.

Since $Q_{\lambda}(t, x)=\frac{4 \lambda}{1+\left(\lambda x-\lambda^{2} t\right)^{2}}$, we set $U_{0}^{(2)}(t, x)=2 \arctan \left(\lambda x-\lambda^{2} t\right)$. We next recall the equation $u_{0}^{(1)}(t, x)$ verifies:

$$
\partial_{t}\left(\frac{1}{2} u_{0}^{(1)}\right)+\mathcal{H} \partial_{x}^{2}\left(\frac{1}{2} u_{0}^{(1)}\right)+\partial_{x}\left(Q_{\lambda} \frac{1}{2} u_{0}^{(1)}\right)+b \partial_{x}\left(\left(\frac{1}{2} u_{0}^{(1)}\right)^{2}\right)=0 .
$$

We then define first $U_{0}^{(1)}(t, 0)$ by $U_{0}^{(1)}(0,0)=0$ and

$$
\partial U_{0}^{(1)}(t, 0)+\mathcal{H} \partial_{x}\left(\frac{1}{2} u_{0}^{(1)}(t, 0)\right)+Q_{\lambda}(t, 0) \frac{1}{2} u_{0}^{(1)}(t, 0)+b\left(\frac{1}{2} u_{0}^{(1)}(t, 0)\right)^{2}=0 .
$$

We then construct $U_{0}^{(1)}(t, x)$ by $\partial_{x} U_{0}^{(1)}(t, x)=\frac{1}{2} u_{0}^{(1)}(t, x)$. Notice that $U_{0}^{(1)}$ is real-valued. Using the equation for $u_{0}^{(1)}$, we have

$$
\partial_{x}\left(\partial_{t} U_{0}^{(1)}+\mathcal{H} \partial_{x}^{2} U_{0}^{(1)}+Q_{\lambda} \partial_{x} U_{0}^{(1)}+b\left(\partial_{x} U_{0}^{(1)}\right)^{2}\right)=0 \quad \text { on } \mathbb{R} \times[-2,2] .
$$


But then, on $\mathbb{R} \times[-2,2]$, we have

$$
\partial_{t} U_{0}^{(1)}(t, x)+\frac{1}{2} \mathcal{H} \partial_{x} u_{0}^{(1)}(t, x)+Q_{\lambda}(t, x) \frac{1}{2} u_{0}^{(1)}(t, x)+\frac{b}{4}\left(u_{0}^{(1)}(t, x)\right)^{2} .
$$

We then define $U_{0}(t, x)=b U_{0}^{(1)}(t, x)+U_{0}^{(2)}(t, x)$, and all our properties hold. We recall that

$$
\left\{\begin{array}{l}
\widetilde{u}_{t}+\mathcal{H} \widetilde{u}_{x x}+\left(u_{0} \widetilde{u}\right)_{x}+b\left(\frac{1}{2} \widetilde{u}^{2}\right)_{x}=0, \\
\widetilde{u}_{\mid t=0}=P_{+\operatorname{high}} \phi+P_{-\operatorname{high}} \phi .
\end{array}\right.
$$

We now proceed as in Section 2 of [11]. We define

$$
P_{\text {+high }} \widetilde{u}=e^{-i U_{0}} w_{+}, \quad P_{\text {-high }} \widetilde{u}=e^{i U_{0}} w_{-} \quad \text { and } \quad P_{\text {low }} \widetilde{u}=w_{0} .
$$

Applying $P_{\text {+high }}, P_{- \text {high }}, P_{\text {low }}$ to the above equation and using the definitions above, we have (we write the equation for $w_{+}$, the one for $w_{-}$is analogous, the one for $w_{0}$ will be written later). Following the argument in [11], one gets:

$$
\begin{aligned}
& \left(w_{+}\right)_{t}+\mathcal{H} \partial_{x}^{2} w_{+}=-\frac{b}{2} e^{i U_{0}} P_{+ \text {high }} \partial_{x}\left(\left(e^{-i U_{0}} w_{+}+e^{i U_{0}} w_{-}+w_{0}\right)^{2}\right) \\
& -e^{-i U_{0}} P_{+ \text {high }} \partial_{x}\left(u_{0}\left(e^{i U_{0}} w_{-}+w_{0}\right)\right)+e^{i U_{0}}\left(P_{- \text {high }}+P_{\text {low }}\right)\left(e^{i U_{0}} u_{0} \partial_{x} w_{+}\right) \\
& +2 i P_{-} \partial_{x}^{2} w_{+}-e^{i U_{0}} P_{+ \text {high }}\left(\partial_{x}\left(u_{0} e^{-i U_{0}} w_{+}\right)\right)+i w_{+}\left[\left(U_{0}\right)_{t}-i\left(U_{0}\right)_{x x}-\left(\left(U_{0}\right)_{x}\right)^{2}\right],
\end{aligned}
$$

and so after more calculations, we get

$$
\begin{aligned}
\left(w_{+}\right)_{t}+\mathcal{H} \partial_{x}^{2} w_{+}= & -\frac{b}{2} e^{i U_{0}} P_{+ \text {high }} \partial_{x}\left(\left(e^{-i U_{0}} w_{+}+e^{i U_{0}} w_{-}+w_{0}\right)^{2}\right) \\
& -e^{-i U_{0}} P_{\text {+high }}\left[\partial_{x}\left(u_{0} P_{- \text {high }}\left(e^{i U_{0}} w_{-}\right)+u_{0} P_{\text {low }}\left(w_{0}\right)\right)\right] \\
& +e^{i U_{0}}\left(P_{- \text {high }}+P_{\text {low }}\right)\left[\partial_{x}\left(u_{0} P_{+ \text {high }}\left(e^{-i U_{0}} w_{+}\right)\right)\right] \\
& +2 i P_{-}\left[\partial_{x}^{2}\left(e^{i u_{0}} P_{+ \text {high }}\left(e^{-i U_{0}} w_{+}\right)\right)\right] \\
& +i w_{+}\left[\left(U_{0}\right)_{t}+\mathcal{H} \partial_{x}^{2} U_{0}+\left(\partial_{x} U_{0}\right)^{2}+i P_{+} \partial_{x} U_{0}\right]
\end{aligned}
$$

We recall $\partial_{x} U_{0}^{(2)}=\frac{1}{2} Q_{\lambda}$ and that $Q_{\lambda}$ solves $\partial_{t} Q_{\lambda}+\mathcal{H} \partial_{x}^{2} Q_{\lambda}+\partial_{x}\left(\frac{1}{2} Q_{\lambda}^{2}\right)=0$ or $\partial_{t} U_{0}^{(2)}+\mathcal{H} \partial_{x}^{2} U_{0}^{(2)}=-\frac{1}{4} Q_{\lambda}^{2}$ and $\partial_{t} U_{0}^{(1)}+\mathcal{H} \partial_{x}^{2} U_{0}^{(1)}=-Q_{\lambda} \partial U_{0}^{(1)}-b\left(\partial_{x} U_{0}^{(1)}\right)^{2}$. Hence, $\partial_{t} U_{0}+\mathcal{H} \partial_{x}^{2} U_{0}+\left(\partial_{x} U_{0}\right)^{2}=0$ and we get $\partial w_{+}+\mathcal{H} w_{+}=E_{+}\left(w_{+}, w_{-}, w_{0}\right)$, where $E_{+}$is defined as in [11, p. 756], except that the first term is multiplied now by $b$. The equation for $w_{-}$and $E_{-}$is similar. The equation for $w_{0}$ writes

$$
\partial_{t}\left(P_{\text {low }} \widetilde{u}\right)+\mathcal{H} \partial_{x}^{2} P_{\text {low }} \widetilde{u}+P_{\text {low }} \partial_{x}\left(u_{0} \widetilde{u}\right)+\frac{b}{2} P_{\text {low }} \partial_{x}\left((\widetilde{u})^{2}\right)=0,
$$

where $\widetilde{u}=e^{-i U_{0}} w_{+}+e^{i U_{0}} w_{-}+w_{0}$. 
Next, we note that, with $\delta=\left(\lambda_{0}^{\frac{1}{2}}+b_{0}\right)$, the estimates (10.19) in [11] hold. Because of this and the form of $E_{+}, E_{-}, E_{0}$, just as in Proposition 10.5 in [11], we have

$$
\begin{aligned}
\| \psi(t)(\mathbf{E}(\mathbf{w})- & \left.\mathbf{E}\left(\mathbf{w}^{\prime}\right)\right)\left\|_{N^{\sigma}} \leq C b_{0}\right\| \mathbf{w}-\mathbf{w}^{\prime} \|_{F^{\sigma}}\left(\|\mathbf{w}\|_{F^{0}}+\left\|\mathbf{w}^{\prime}\right\|_{F^{0}}\right) \\
& +C b_{0}\left\|\mathbf{w}-\mathbf{w}^{\prime}\right\|_{F^{0}}\left(\|\mathbf{w}\|_{F^{\sigma}}+\left\|\mathbf{w}^{\prime}\right\|_{F^{\sigma}}\right)+C \delta\left\|\mathbf{w}-\mathbf{w}^{\prime}\right\|_{F^{\sigma}} .
\end{aligned}
$$

Note that $\mathbf{w}=\left(w_{+}, w_{-}, w_{0}\right)$ and

$$
\mathbf{E}(\mathbf{w})=\left(E_{+}\left(w_{+}, w_{-}, w_{0}\right), E_{-}\left(w_{+}, w_{-}, w_{0}\right), E_{0}\left(w_{+}, w_{-}, w_{0}\right)\right)
$$

as in [11]. The rest of the notation (the norm $\|\cdot\|_{N^{\sigma}}$ and the function $\psi$ ) is also taken from [11]. We have a slightly different formula for $E_{0}$, but (10.27) in [11] gives the estimate in our case also.

We then construct a solution to

$$
\left\{\begin{array}{l}
\mathbf{v}_{t}+\mathcal{H} \mathbf{v}_{x x}=\mathbf{E}(\mathbf{v}) \quad \text { on } \mathbb{R} \times\left[-\frac{5}{4}, \frac{5}{4}\right] \\
\mathbf{v}(0)=\Phi
\end{array}\right.
$$

as in (10.32)-(10.37) in [11]. Note that (10.35) and $\left\|v(\Phi)-v\left(\Phi^{\prime}\right)\right\|_{F^{0}\left(\left[-\frac{5}{4}, \frac{5}{4}\right]\right)} \leq$ $C\left\|\Phi-\Phi^{\prime}\right\|_{\widetilde{H}^{0}}$ hold here too. Next, with

$$
\Phi=\left(\phi_{+}, \phi_{-}, \phi_{0}\right)=\left(e^{i U_{0}(0, .)} P_{\text {+high }} \phi, e^{-i U_{0}(0, .)} P_{- \text {high }} \phi, 0\right),
$$

$\Phi \in \widetilde{H}^{20}$, by Lemma 10.1 in [11].

We next show $\left(w_{+}, w_{-}, w_{0}\right)=\mathbf{v}(\Phi)$ in $\mathbb{R} \times[-1,1]$. This is as in [11]. Proposition 8, and the second estimate in Proposition 9 now follow from the bounds on $\mathbf{v}(\Phi)$ i.e. (10.35). For Proposition 9, note that for $N$ large, $U_{0}$ corresponding to $\phi$ and to $\phi_{N}$ defined by $\hat{\phi}_{N}=\mathbf{1}_{[-N, N]}(\xi) \hat{\phi}(\xi)$ are the same. We then have

$$
u(t, x)=u_{0}^{(1)}+u-u_{0}^{(1)}=u_{0}^{(1)}+\widetilde{u}=u_{0}^{(1)}+e^{-i U_{0}} w_{+}+e^{i U_{0}} w_{-}+w_{0}
$$

and similarly,

$$
u^{N}(t, x)=u_{0}^{(1)}+u^{N}-u_{0}^{(1)}=u_{0}^{(1)}+e^{-i U_{0}} w_{+}^{N}+e^{i U_{0}} w_{-}^{N}+w_{0}^{N} .
$$

Hence,

$$
\begin{aligned}
\sup _{t \in[-1,1]}\left\|u(t, .)-u^{N}(t, .)\right\|_{L^{2}} & \leq \sup _{t \in[-1,1]}\left\|w(t)-w^{N}(t)\right\|_{L^{2}} \\
& \leq C\left\|\psi(t)\left[w-w^{N}\right]\right\|_{F^{0}} \leq C\left\|\phi-\phi^{N}\right\|_{L^{2}}
\end{aligned}
$$

as desired, giving Proposition 9. 


\section{A. Appendix}

First, we recall the following inequalities:

\section{Lemma 14.}

$$
\begin{gathered}
\forall 2 \leq p<+\infty, \quad\|f\|_{L^{p}} \leq C_{p}\|f\|_{L^{2}}^{\frac{2}{p}}\left\|D^{\frac{1}{2}} f\right\|_{L^{2}}^{\frac{p-2}{p}} \\
\|D(f g)-g D f\|_{L^{2}} \leq C\|f\|_{L^{4}}\|D g\|_{L^{4}} \\
\left\|D^{\frac{1}{2}}(f g)-g D^{\frac{1}{2}} f\right\|_{L^{2}} \leq C\|f\|_{L^{4}}\left\|D^{\frac{1}{2}} g\right\|_{L^{4}} .
\end{gathered}
$$

Recall that (A.1) is the Gagliardo-Nirenberg inequality, which follows from complex interpolation and Sobolev embedding.

Estimate (A.2) is due to Calderón [6], see also Coifman and Meyer [7], formula (1.1).

Estimate (A.3) is a consequence of Theorem A.8 in [13] for functions depending only on $x$, with the following choice of parameters: $\alpha=\frac{1}{2}, \alpha_{1}=0$, $\alpha_{2}=\frac{1}{2}, p=2, p_{1}=p_{2}=4$.

\section{A.1. Proof of (2.15)}

We claim that for a function $u(x)$ fixed in $H^{2}(\mathbb{R})$

$$
\int_{y=0} \partial_{y}\left(U^{2}\right) \Phi=-2 \iint_{\mathbb{R}_{+}^{2}}|\nabla U|^{2} \Phi+\int_{y=0} U^{2} \partial_{y} \Phi
$$

where $U(x, y)$ is the harmonic extension of $u(x)$ in $\mathbb{R}_{+}^{2}$ and $\Phi(x, y)$ is defined in $(2.14)$.

First, we observe that

$$
U, \nabla U \in L^{\infty}\left(\mathbb{R}_{+}^{2}\right) \quad \text { and } \quad \sup _{y>0}|U(x, y)| \rightarrow 0 \text { as }|x| \rightarrow+\infty
$$

Indeed, from [26], Theorem 1, p. 62, we have $\sup _{y>0}|U(x, y)| \leq M u(x)$, where $M u(x)$ is the maximal function of $u$ (see [26] Chapter 1), and similarly, $\sup _{y>0}\left|\partial_{x} U(x, y)\right| \leq M u_{x}(x), \sup _{y>0}\left|\partial_{y} U(x, y)\right| \leq M\left(\mathcal{H} u_{x}\right)(x)$. Moreover, from [26] Theorem 1, p. 5, since $u, u_{x}, \mathcal{H} u_{x} \in H^{1} \subset L^{\infty}$, we obtain $M u, M u_{x}, M\left(H u_{x}\right) \in L^{\infty}$. Finally, since $u \in H^{1}$, we have $|u(x)| \rightarrow 0$ as $|x| \rightarrow+\infty$, which implies by the definition of the maximal function (see [26, page 4]) that $M u(x) \rightarrow 0$ as $|x| \rightarrow+\infty$. Thus (A.5) is proved.

Let $R>0$. We use the Green formula on $D_{R}^{+}=\left\{(x, y) \in \mathbb{R}_{+}^{2} \mid x^{2}+y^{2}<\right.$ $\left.R^{2}\right\}$. Let $\Gamma_{R}^{+}=\left\{(x, y) \in \mathbb{R}_{+}^{2} \mid x^{2}+y^{2}=R^{2}\right\}$ and $I_{R}=(x, 0) \mid x \in[-R, R]$. 
Then:

$$
\begin{aligned}
\int_{\Gamma_{R}^{+} \cup I_{R}} \partial_{n}\left(U^{2}\right) \Phi & =-\iint_{D_{R}^{+}}\left(\Delta U^{2}\right) \Phi+\iint_{D_{R}^{+}} U^{2} \Delta \Phi+\int_{\Gamma_{R}^{+} \cup I_{R}} U^{2} \partial_{n} \Phi \\
& =-2 \iint_{D_{R}^{+}}|\nabla U|^{2} \Phi+\int_{\Gamma_{R}^{+} \cup I_{R}} U^{2} \partial_{n} \Phi
\end{aligned}
$$

where $\partial_{n}$ denotes the inward normal derivative since $\Delta \Phi=0$ and $\Delta U^{2}=$ $2|\nabla U|^{2}$. Therefore, we only have to prove the following convergence results:

$$
\begin{aligned}
& \text { (A.7) } \lim _{R \rightarrow+\infty} \int_{\Gamma_{R}^{+}} \partial_{n}\left(U^{2}\right) \Phi=0, \lim _{R \rightarrow+\infty} \int_{I_{R}} \partial_{n}\left(U^{2}\right) \Phi=\int_{y=0} \partial_{y}\left(U^{2}\right) \Phi=2 \int\left(\mathcal{H} u_{x}\right) u \varphi^{\prime} \\
& \text { (A.8) } \lim _{R \rightarrow+\infty} \int_{\Gamma_{R}^{+}} U^{2} \partial_{n} \Phi=0, \quad \lim _{R \rightarrow+\infty} \int_{I_{R}} U^{2} \partial_{n} \Phi=\int_{y=0} U^{2} \partial_{y} \Phi=\int u^{2}\left(\mathcal{H} \varphi^{\prime \prime}\right) .
\end{aligned}
$$

The $\operatorname{limits} \lim _{R \rightarrow+\infty} \int_{-R}^{R}\left(\mathcal{H} u_{x}\right) u \varphi^{\prime}=\int\left(\mathcal{H} u_{x}\right) u \varphi^{\prime}$ and

$$
\lim _{R \rightarrow+\infty} \int_{-R}^{R} u^{2}\left(\mathcal{H} \varphi^{\prime \prime}\right)=\int u^{2}\left(\mathcal{H} \varphi^{\prime \prime}\right)
$$

are clear since $u \in H^{1}$. Next, from the expression of $\Phi(x, y)$ in (2.14), we have $\Phi(x, y) \leq C(1+y) R^{-2}$ on $\Gamma_{R}^{+}$. Therefore, from (A.5), (do denotes the unit lenght element on $\left.\Gamma_{R}^{+}\right)$

$$
\begin{aligned}
\int_{\Gamma_{R}^{+}}\left|\partial_{n}\left(U^{2}\right) \Phi\right| & \leq \frac{1}{R^{2}} \int_{\Gamma_{R}^{+}}|\nabla U||U|(1+y) d \sigma \\
& \leq \frac{C}{R^{2}} \int_{\Gamma_{R}^{+} \cap\{|x| \leq \sqrt{R}\}}(1+y) d \sigma+C \sup _{|x|>\sqrt{R}, y>0}|U(x, y)| \\
& \leq \frac{C}{\sqrt{R}}+C \sup _{|x|>\sqrt{R}, y>0}|U(x, y)|
\end{aligned}
$$

and so (A.7) is proved. Estimate (A.8) is proved similarly and is easier since $\partial_{y} \Phi$ has more decay than $\Phi$.

\section{A.2. Proof of (2.30)}

In the proof of (2.30), the time $t$ is fixed, so we set $y_{0}=x_{0}+\lambda\left(t_{0}-t\right)$.

Let $\chi: \mathbb{R} \rightarrow \mathbb{R}$ be a $C^{\infty}$ function such that $\chi=1$ on $[0,1], \chi=0$ on $(-\infty,-1] \cap[2,+\infty)$ and $\chi \leq 1$ on $\mathbb{R}$. Let $\chi_{n}(x)=\chi(x-n)$. Then, by the 
Gagliardo-Nirenberg inequality (A.1), we obtain

$$
\begin{aligned}
\int|\eta|^{3} \varphi^{\prime}\left(x-y_{0}\right) & \leq \sum_{n \in \mathbb{Z}} \int_{n}^{n+1}|\eta|^{3} \varphi^{\prime}\left(x-y_{0}\right) \leq \sum_{n \in \mathbb{Z}}\left(\int|\eta|^{3} \chi_{n}^{3}\right) \sup _{\left[n-y_{0}, n+1-y_{0}\right]} \varphi^{\prime} \\
& \leq \sum_{n \in \mathbb{Z}}\left(\int\left|D^{\frac{1}{2}}\left(\eta \chi_{n}\right)\right|^{2}\right)^{\frac{1}{2}}\left(\int\left(\eta \chi_{n}\right)^{2}\right)_{\left[n-y_{0}, n+1-y_{0}\right]} \varphi^{\prime} .
\end{aligned}
$$

By Lemma 14 and (2.2), we get

$$
\left\|D^{\frac{1}{2}}\left(\eta \chi_{n}\right)\right\|_{L^{2}} \leq C\left\|\left(D^{\frac{1}{2}} \eta\right) \chi_{n}\right\|_{L^{2}}+C\|\eta\|_{L^{4}}\left\|D^{\frac{1}{2}} \chi_{n}\right\|_{L^{4}} \leq C\|\eta\|_{H^{\frac{1}{2}}} \leq C \alpha_{0} .
$$

Thus,

$$
\int|\eta|^{3} \varphi^{\prime}\left(x-y_{0}\right) \leq C \alpha_{0} \sum_{n \in \mathbb{Z}}\left(\int\left(\eta \chi_{n}\right)^{2}\right) \sup _{\left[n-y_{0}, n+1-y_{0}\right]} \varphi^{\prime} \leq C \alpha_{0} \int \eta^{2} \varphi^{\prime}\left(x-y_{0}\right)
$$

by the properties of $\chi$ and the following elementary remark:

$$
\forall y \in \mathbb{R}, \quad \sup _{[y, y+4]} \varphi^{\prime} \leq C \inf _{[y, y+4]} \varphi^{\prime} .
$$

Note that the constant $C$ is independent of $A$, for $A>1$.

\section{A.3. Properties of the operator $\mathcal{L}$}

We recall from [29]-[31] and [3] the following properties of $\mathcal{L}$ (recall $\mathcal{L} \eta=$ $\left.-\mathcal{H} \eta_{x}+\eta-Q \eta\right)$.

Lemma 15. The operator $\mathcal{L}$ is self-adjoint on $L^{2}$ and satisfies the following properties.

(i) The operator $\mathcal{L}$ has exactly one negative eigenvalue $\lambda_{0}$ of multiplicity 1 with corresponding eigenfunction $f_{0}$, which can be chosen so that $f_{0}>0$.

(ii) $\operatorname{Ker} \mathcal{L}=\operatorname{span}\left\{Q^{\prime}\right\}$.

(iii) There exists $\lambda>0$ such that, for all $z \in H^{\frac{1}{2}}$,

$$
(z, Q)=\left(z, Q^{\prime}\right)=0 \quad \Rightarrow \quad(\mathcal{L} z, z) \geq \lambda(z, z) .
$$

Remark 6. Recall from Bennett et al. ([3], Appendix B) that the spectrum of $\mathcal{L}$ is completely understood. Indeed, the operator $\mathcal{L}$ has exactly four eigenvalues, $\lambda_{0}=-\frac{1}{2}(1+\sqrt{5}), 0, \frac{1}{2}(-1+\sqrt{5}), 1$ and a continuous spectrum $[1,+\infty)$.

Now, we sketch a proof of Lemma 15 using general arguments from [29]-[31]. 
Sketch of proof. One easily checks that $\mathcal{L} Q^{\prime}=0$ (differentiate the equation of $Q\left(x+x_{0}\right)$ with respect to $x_{0}$ and take $\left.x_{0}=0\right)$, and that $\mathcal{L} f_{0}=-\lambda_{0} f_{0}$, where $f_{0}=Q+\frac{1}{4}(1+\sqrt{5}) Q^{2}$ (by (3.35)). Moreover, the proof of (i) follows from the variational characterization of $Q$, see Proposition 4.2 of [31]. Recall that $\frac{d}{d c} \int Q_{c}^{2}=\int Q^{2}>0$ (subcriticality) implies that $\inf \{(\mathcal{L} f, f) ;(f, Q)=0$, $\left.\|f\|_{L^{2}}=1\right\}=0$ (see proof of Proposition 5.1 in [31] and Proposition 3.1 in [30]).

Now, we give a new proof for (ii). Let $f \in L^{2}$ be such that $\mathcal{L} f=0$. First, we remark that $f \in H^{s}$, for all $s \geq 0$. Moreover, by similar estimates as in [1], we have $|f(x)| \leq \frac{C}{1+x^{2}}$. Integrating $\mathcal{L} f=0$ on $\mathbb{R}$, we obtain $\int(f-f Q)=0$. But, we also have $(f, Q)=-(f, \mathcal{L} S)=-(\mathcal{L} f, S)=0$ (see (3.35)). Thus, $\int f=0$ and we can define $g(x)=\int_{-\infty}^{x} f(s) d s \in L^{2}$, which satisfies $\mathcal{L}\left(g^{\prime}\right)=0$. Let now $\widetilde{g}=g-a Q$ be such that $(\widetilde{g}, Q)=0$ and $\mathcal{L}\left(\widetilde{g}^{\prime}\right)=0$. From (3.12) and (3.36), we obtain $\int\left|D^{\frac{1}{2}} \widetilde{g}\right|^{2}+(\mathcal{L} \widetilde{g}, \widetilde{g}) \leq 0$. But, since $(\widetilde{g}, Q)=0$, we have $(\mathcal{L} \widetilde{g}, \widetilde{g}) \geq 0$. Thus, $\int\left|D^{\frac{1}{2}} \widetilde{g}\right|^{2}=0$ and $\widetilde{g} \equiv 0$, so that $g=a Q$ and $f=a Q^{\prime}$.

Finally, we sketch the proof of (iii), which follows from the arguments of the proof of Proposition 2.9 in [29] (see also Section 6, example 4 in [31]). By contradiction, assuming that

$$
\inf \left\{(\mathcal{L} f, f) ;(f, Q)=\left(f, Q^{\prime}\right)=0,\|f\|_{L^{2}}=1\right\}=0,
$$

and using compactness arguments as in Proposition 2.9 in [29], we obtain the existence of $f \in H^{\frac{1}{2}}, \lambda, \beta, \gamma \in \mathbb{R}$ (Lagrange multipliers) such that

$$
(\mathcal{L} f, f)=0, \quad(\mathcal{L}-\lambda) f=\beta Q+\gamma Q^{\prime}, \quad(f, Q)=\left(f, Q^{\prime}\right)=0, \quad\|f\|_{L^{2}}=1 .
$$

But, taking the scalar product by $f$, we find $\lambda=0$. Then, taking the scalar product by $Q^{\prime}$, we find $\gamma=0$. Taking the scalar product with $S$ (see (3.35)), using $(S, Q)=\frac{1}{2}(Q, Q)$ and $\mathcal{L}(S)=-Q$, we find $\beta=0$, so that $\mathcal{L} f=0$ and $\left(f, Q^{\prime}\right)=0$. This implies $f=0$ by (ii), a contradiction.

\section{References}

[1] Amick, C. J. AND Toland, J. F.: Uniqueness and related analytic properties for the Benjamin-Ono equation - a nonlinear Neumann problem in the plane. Acta Math. 167 (1991), 107-126.

[2] Benjamin, T. B.: Internal waves of permanent form in fluids of great depth. Journal of Fluid Mechanics 29 (1967), 559-592.

[3] Bennett, D. P., Brown, R. W., Stansfield, S. E., Stroughair, J. D. And Bona, J. L.: The stability of internal solitary waves. Math. Proc. Cambridge Philos. Soc. 94 (1983), 351-379.

[4] Bona, J. L., Souganidis, P.E. and Strauss, W. A.: Stability and instability of solitary waves of Korteweg-de Vries type. Proc. Roy. Soc. London Ser. A 411 (1987), 395-412. 
[5] Burq, N. and Planchon, F.: On well-posedness for the Benjamin-Ono equation. Math. Ann. 340 (2008), no. 3, 497-542.

[6] Calderón, A.-P.: Commutators of singular integral operators. Proc. Nat. Acad. Sci. U.S.A. 53 (1965), 1092-1099.

[7] Coifman, R. R. And Meyer, Y.: On commutators of singular integrals and bilinear singular integrals. Trans. Amer. Math. Soc. 212 (1975), $315-331$.

[8] Herr, S., Ionescu, A. D., Kenig, C. E. And Koch, H.: Global solutions to dispersive nonlinear equations. Preprint.

[9] Ginibre, J. And Velo, G.: Commutator expansions and smoothing properties of generalized Benjamin-Ono equations. Ann. Inst. H. Poincaré Phys. Théor. 51 (1989), 221-229.

[10] Gustafson, S., Takaoka, H. and Tsai, T.-P.: Stability in $H^{\frac{1}{2}}$ of the sum of $K$ solitons for the Benjamin-Ono equation. J. Math. Phys. 50 (2009), no. $1,013101,14 \mathrm{pp}$.

[11] Ionescu, A. D. And Kenig, C. E.: Global well-posedness of the Benjamin-Ono equation in low-regularity spaces. J. Amer. Math. Soc. 20 (2007), 753-798.

[12] Kato, T.: On the Cauchy problem for the (generalized) Korteweg-de Vries equation. In Studies in applied mathematics, 93-128. Adv. Math. Suppl. Stud. 8, Academic Press, New York, 1983.

[13] Kenig, C. E. , Ponce, G. And Vega, L.: Well-posedness and scattering results for the generalized Korteweg-de Vries equation via the contraction principle. Comm. Pure Appl. Math. 46 (1993), 527-620.

[14] Martel, Y.: Linear problems related to asymptotic stability of solitons of the generalized KdV equations. SIAM J. Math. Anal. 38 (2006), 759-781.

[15] Martel, Y. And Merle, F.: Instability of solitons for the critical generalized Korteweg-de Vries equation. Geom. Funct. Anal. 11 (2001), 74-123.

[16] Martel, Y. and Merle, F.: A Liouville theorem for the critical generalized Korteweg-de Vries equation. J. Math. Pures Appl. (9) 79 (2000), $339-425$.

[17] Martel, Y. And Merle, F.: Asymptotic stability of solitons for subcritical generalized KdV equations. Arch. Ration. Mech. Anal. 157 (2001), $219-254$

[18] Martel, Y. and Merle, F.: Asymptotic stability of solitons of the subcritical gKdV equations revisited. Nonlinearity 18 (2005), no. 1, 55-80.

[19] Martel, Y. And Merle, F.: Asymptotic stability of solitons of the gKdV equations with a general nonlinearity. Math. Ann. 341 (2008), 391-427.

[20] Martel, Y. and Merle, F.: Refined asymptotics around soliton for gKdV equations. Discrete Contin. Dyn. Syst. 20 (2008), 177-218.

[21] Martel, Y., Merle, F. And Tsai, T.-P.: Stability and asymptotic stability in the energy space of the sum of $N$ solitons for the subcritical gKdV equations. Comm. Math. Phys. 231 (2002), 347-373. 
[22] Matsuno, Y.: The Lyapunov stability of the $N$-soliton solutions in the Lax hierarchy of the Benjamin-Ono equation. J. Math. Phys. 47 (2006), 103505, 13pp.

[23] Neves, A. And Lopes, O.: Orbital stability of double solitons for the Benjamin-Ono equation. Comm. Math. Phys. 262 (2006), 757-791.

[24] Pego, R. L. And Weinstein, M. I.: Asymptotic stability of solitary waves. Comm. Math. Phys. 164 (1994), 305-349.

[25] Ponce, G.: Smoothing properties of solutions to the Benjamin-Ono equation. In Analysis and partial differential equations, 667-679. Lecture Notes in Pure and Appl. Math. 122. Dekker, New York, 1990.

[26] Stein, E.: Singular integrals and differentiability properties of functions. Princeton Mathematical Series 30. Princeton Univ. Press, Princeton, 1970.

[27] TAO, T.: Global well-posedness of the Benjamin-Ono equation in $H^{1}(\mathbb{R})$. J. Hyperbolic Differ. Equ. 1 (2004), 27-49.

[28] Toland, J.F.: The Peierls-Nabarro and Benjamin-Ono equations. J. Funct. Anal. 145 (1997), 136-150.

[29] Weinstein, M. I.: Modulational stability of ground states of nonlinear Schrödinger equations. SIAM J. Math. Anal. 16 (1985), 472-491.

[30] Weinstein, M. I.: Lyapunov stability of ground states of nonlinear dispersive evolution equations. Comm. Pure Appl. Math. 39 (1986), 51-67.

[31] Weinstein, M. I.: Existence and dynamic stability of solitary wave solutions of equations arising in long wave propagation. Comm. Partial Differential Equations 12 (1987), 1133-1173.

Recibido: 2 de enero de 2008

Revisado: 22 de mayo de 2008

Carlos E. Kenig Department of Mathematics, University of Chicago 5734 University Ave. Chicago, Illinois 60637-1514, USA cek@math .uchicago.edu

Yvan Martel

Université de Versailles Saint-Quentin-en-Yvelines, Mathématiques 45, Av. des Etats-Unis 78035 Versailles cedex, France martel@math.uvsq.fr

The first author is partly supported by the NSF grant DMS-0456583. This work was initiated when the second author was visiting the University of Chicago. He would like to thank the Department of Mathematics for its hospitality. The second author is partly supported by the Agence Nationale de la Recherche (ANR ONDENONLIN). 